\title{
A Comparison of Young Star Properties with Local Galactic Environment for LEGUS/ LITTLE THINGS Dwarf Irregular Galaxies
}

Deidre A. Hunter ${ }^{1}$ (D), Angela Adamo ${ }^{2}$ (D), Bruce G. Elmegreen ${ }^{3}$ (D), Samavarti Gallardo ${ }^{1,4}$, Janice C. Lee ${ }^{5}$ (D), David O. Cook ${ }^{6}$ (ID), David Thilker $^{7}$ (D), Bridget Kayitesi ${ }^{7,8}$, Hwihyun Kim $^{9}$ (D) , Lauren Kahre ${ }^{10}$, Leonardo Ubeda ${ }^{5}$, Stacey N. Bright ${ }^{5}$, Jenna E. Ryon ${ }^{5}$, Daniela Calzetti $^{11}$ (D), Monica Tosi ${ }^{12}$ (D), Kathryn Grasha ${ }^{11}$ (D), Matteo Messa ${ }^{2}$, Michele Fumagalli ${ }^{13}$ (D), Daniel A. Dale ${ }^{14}$ (D),

Elena Sabbi ${ }^{5}$ (D) , Michele Cignoni ${ }^{15,16}$ (D), Linda J. Smith ${ }^{17}$ (D), Dimitrios M. Gouliermis ${ }^{18,19}$ (iD), Eva K. Grebel ${ }^{20}$ (iD), Alessandra Aloisi ${ }^{5}$ (D), Bradley C. Whitmore ${ }^{5}$, Rupali Chandar ${ }^{21}$ (iD), and Kelsey E. Johnson ${ }^{22}$ (D)

${ }^{1}$ Lowell Observatory, 1400 West Mars Hill Road, Flagstaff, AZ 86001, USA

${ }^{2}$ Department of Astronomy, The Oskar Klein Centre, Stockholm University, Stockholm, Sweden

${ }^{3}$ IBM Research Division, T.J. Watson Research Center, Yorktown Heights, NY 10598, USA

${ }^{4}$ Northern Arizona University, Flagstaff, AZ 86011, USA

${ }^{5}$ Space Telescope Science Institute, 3700 San Martin Drive, Baltimore, MD 21218, USA

${ }^{6}$ Department of Astronomy and Astrophysics, California Institute of Technology, Pasadena, CA 91125, USA

${ }^{7}$ Department of Physics and Astronomy, Johns Hopkins University, Baltimore, MD, USA ${ }^{8}$ Hamilton College, 198 College Hill Road, Clinton, NY 13323, USA

${ }^{9}$ Gemini Observatory, Casilla 603, La Serena, Chile

${ }^{10}$ Department of Astronomy, New Mexico State University, Las Cruces, NM, USA

${ }^{11}$ Department of Astronomy, University of Massachusetts-Amherst, Amherst, MA 01003, USA

${ }^{12}$ INAF-Osservatorio di Astrofisica e Scienza dello Spazio, Bologna, Italy

${ }^{13}$ Institute for Computational Cosmology and Centre for Extragalactic Astronomy, Durham University, Durham, UK

${ }_{15}^{14}$ Department of Physics and Astronomy, University of Wyoming, Laramie, WY, USA

${ }^{15}$ Department of Physics, University of Pisa, Largo B. Pontecorvo 3, I-56127, Pisa, Italy

${ }^{16}$ INFN, Largo B. Pontecorvo 3, I-56127, Pisa, Italy

${ }^{17}$ European Space Agency/Space Telescope Science Institute, Baltimore, MD 21218, USA

${ }^{18}$ Zentrum für Astronomie der Universität Heidelberg, Institut für Theoretische Astrophysik, Albert-Ueberle-Str. 2, D-69120 Heidelberg, Germany

${ }^{19}$ Max Planck Institute for Astronomy, Königstuhl 17, D-69117 Heidelberg, Germany

${ }^{20}$ Astronomisches Rechen-Institut, Zentrum für Astronomie der Universität Heidelberg, Mönchhofstr. 12-14, D-69120 Heidelberg, Germany

${ }^{21}$ Department of Physics and Astronomy, The University of Toledo, Toledo, OH 43606, USA

${ }^{22}$ Department of Astronomy, University of Virginia, Charlottesville, VA 22904, USA

Received 2017 November 21; revised 2018 May 2; accepted 2018 May 13; published 2018 June 21

\begin{abstract}
We have explored the role environmental factors play in determining characteristics of young stellar objects in nearby dwarf irregular and blue compact dwarf galaxies. Star clusters are characterized by concentrations, masses, and formation rates; $\mathrm{OB}$ associations by mass and mass surface density; $\mathrm{O}$ stars by their numbers and nearultraviolet absolute magnitudes; and $\mathrm{H}$ II regions by $\mathrm{H} \alpha$ surface brightnesses. These characteristics are compared to surrounding galactic pressure, stellar mass density, H I surface density, and star formation rate (SFR) surface density. We find no trend of cluster characteristics with environmental properties, implying that larger-scale effects are more important in determining cluster characteristics or that rapid dynamical evolution erases any memory of the initial conditions. On the other hand, the most massive OB associations are found at higher pressure and $\mathrm{HI}$ surface density, and there is a trend of higher $\mathrm{H}$ II region $\mathrm{H} \alpha$ surface brightness with higher pressure, suggesting that a higher concentration of massive stars and gas is found preferentially in regions of higher pressure. At low pressures we find massive stars but not bound clusters and OB associations. We do not find evidence for an increase of cluster formation efficiency as a function of SFR density. However, there is an increase in the ratio of the number of clusters to the number of $\mathrm{O}$ stars with increasing pressure, perhaps reflecting an increase in clustering properties with SFR.
\end{abstract}

Key words: galaxies: dwarf - galaxies: individual (DDO 50, DDO 53, DDO 63, NGC 3738, Haro 29)

Supporting material: animation

\section{Introduction}

Age and mass distribution functions of compact star clusters are similar among different types of galaxies (e.g., Fall \& Chandar 2012; Whitemore 2017). Nevertheless, the products of star formation come with a wide range in numbers of stars and spatial concentration (e.g., Maíz-Apellániz 2001). There are massive, compact, bound clusters, such as the old globular clusters and young super star clusters (e.g., R136 in the LMC and clusters in NGC 1569, NGC 1705, SBS 0335-052, and $\mathrm{He}$ 2-10; O'Connell et al. 1994; Hunter et al. 1996; Johnson et al. 2000; Reines et al. 2008). Yet, spatially large or massive does not always mean spatially concentrated (Kennicutt \& Chu 1988; Hunter 1995), as shown by large loose associations of stars, some of which occupy a large fraction of a dwarf galaxy (e.g., Constellation III in the LMC, IC 10, I Zw 18, and VII Zw 403; Hunter \& Thronson 1995; Dolphin \& Hunter 1998; Lynds et al. 1998; Hunter 2001). At the small end in spatial size or mass, the star formation process also produces both small compact clusters and small associations (e.g., Aversa et al. 2011; Adamo et al. 2017).

The characteristics of natal clouds affect the products of the star formation process. Simulations by Dobbs et al. (2017), for 
example, show that massive, dense, long-lived clouds produce massive clusters and that smaller clusters form in short-lived clouds. Furthermore, clustered star formation occurs in parts of clouds with enhanced turbulence and density, while isolated star formation is found in parts of clouds with subsonic turbulence (Evans 1999). In addition, star formation proceeds faster in higher-density gas, leading to a more narrow age distribution, while there are longer star formation timescales and larger age spreads in low-density regions (Parmentier et al. 2014).

There is also evidence that the star formation rate (SFR) depends on pressure (Blitz \& Rosolowsky 2006) and that largescale galactic conditions affect the star formation products (see, e.g., Whitemore 2017). We see this spectacularly in merging galaxy systems that have produced large numbers of super star clusters (e.g., the Antennae system; Whitmore \& Schweizer 1995). Lada (2010), for example, suggests that triggering cloud formation through processes that increase the pressure, such as shocks, could be significant in interacting galaxies and in early galaxy formation when globular clusters formed. The old halo globular clusters presumably formed at very high pressures (Kruijssen 2015; Elmegreen 2017). In fact, in a study of a giant molecular cloud (GMC) in the Antennae system, Johnson et al. (2015) find that the pressure in the region in which this GMC is embedded is $10^{4}$ times higher than that in a typical galaxy. Swinbank et al. (2010) find that a massive starburst galaxy at $z=2.3$ has star-forming regions with luminosity densities comparable to cores of GMCs but 100 times larger and $10^{7}$ times more luminous than what we see locally. High-pressure regions caused by collision of supershells within a galaxy and external ram pressure stripping can also facilitate star formation (Bernard et al. 2012; Egorov et al. 2017). Furthermore, there is evidence that the fraction of star formation resulting in bound clusters, $\Gamma$, is higher in regions of high SFR density, especially starburst systems (Adamo et al. 2010, 2011; Goddard et al. 2010), although this is not universally agreed on (see Chandar et al. 2015).

Consequences of the environmental conditions on the star formation products may extend to more local regions within galaxies as well. For example, Adamo et al. (2015) found that $\Gamma$ decreases by factors of a few from the center of M83 to the outer disk and varies from region to region within the galaxy with the local SFR density. In addition, the initial cluster mass function (ICMF) steepens in the outer disk as the upper cluster mass limit declines. Changes in $\Gamma$ and the ICMF with radius are consistent with a decrease in gas pressure (Adamo et al. 2015) or, similarly, gas density (Kruijssen 2012). Streaming of gas around bar potentials and piling up at the ends can also be a local factor in creating giant star-forming regions (Elmegreen \& Elmegreen 1980; Renaud et al. 2015). In blue compact dwarfs (BCDs) the migration of gas to the central regions (Simpson \& Gottesman 2000) increases the SFR densities and the local pressure, enhancing the ability to form massive, concentrated star clusters (Hunter \& Elmegreen 2004).

Elmegreen \& Efremov (1997; see also Ashman \& Zepf 2001) have suggested that the dominant factor in determining the kind of unit (bound super star cluster, open clusters, associations) that is formed is pressure. A high-pressure environment facilitates the formation of massive, bound clusters, whether the high pressure is the result of high gas density or large-scale shocks such as in merging galaxies. An equally massive cluster could be born at lower pressure, but it would not be bound. This is consistent with finding super star clusters in starbursts and merging galaxies and not finding them today in galaxies like the Milky Way, where star formation is driven by internal processes. Escala \& Larson (2008), on the other hand, argue that the formation of massive clusters is determined by gravitational instabilities and surface density of gas, and so massive clusters represent the largest scale in galaxies not stabilized by rotation. However, young and old super star clusters and the somewhat less massive populous clusters are found even in some nearby dwarf irregular (dIrr) galaxies, although dIrrs in general have gas densities too low for gravitational instabilities to drive star formation (Bigiel et al. 2010; Elmegreen \& Hunter 2015). On the other hand, these are usually systems in which there is evidence that the formation of massive star clusters is an anomalous event; the galaxies are too small to sample the cluster mass function to the extreme of super star clusters (e.g., O'Connell et al. 1994). In addition, super star clusters are also usually found in a setting of unusually high star formation activity overall, suggesting that some external perturbation has produced large-scale flows (Hunter 1995; Billett et al. 2002).

Given the evidence that the characteristics of the star formation products relate to their local galactic environment, we undertook a study of five dIrr galaxies for which we have catalogs of star clusters and $\mathrm{O}$ star candidates, as well as pressure, stellar mass, integrated $\mathrm{HI}$, and $\mathrm{H} \alpha$ maps of the galaxies. These data allow us to compare the concentrations and masses of young stellar objects with respect to the surrounding pressure, HI surface density, and stellar mass surface density. We use the term "cluster" here as defined by the LEGUS project (Adamo et al. 2017): compact and centrally concentrated sources (class 1 or 2 objects) that could be gravitationally bound systems, as well as objects with asymmetric profiles and multiple peaks on top of diffuse underlying wings (class 3). We also identify larger and looser OB associations in our sample of galaxies, defined by clumping of O stars. Thus, here we are probing the realm of "normal" star formation products, that is, not super star clusters, and examining all types of star formation products, from small compact clusters, to larger $\mathrm{OB}$ associations, to individual $\mathrm{O}$ stars. In addition, we are examining the role local factors play in determining the characteristics of these objects.

In Section 2 we describe the galaxy sample, the data that we worked with, and the way in which we have defined the environment within the galaxies. In Section 3.1 we present the observational results for the star clusters, in Section 3.2 the results for $\mathrm{H}$ II regions, and in Section 3.4 the results for the $\mathrm{O}$ stars, including their characteristics as a function of galactic characteristics and characteristics of regions at different pressures. We also define clusterings of $\mathrm{O}$ stars and discuss the properties of these associations in Section 3.3, and we compare the numbers of clusters and of $\mathrm{O}$ stars by pressure. In Section 4 we summarize our findings.

\subsection{Galaxy Sample}

There are five dIrr galaxies in common between the Hubble Space Telescope (HST) Legacy Extragalactic UV Survey ${ }^{23}$

\footnotetext{
${ }^{23}$ Based on observations obtained with the NASA/ESA Hubble Space Telescope, at the Space Telescope Science Institute, which is operated by the Association of Universities for Research in Astronomy, Inc., under NASA contract NAS5-26555.
} 
Table 1

The Galaxy Sample

\begin{tabular}{|c|c|c|c|c|c|c|c|c|}
\hline Galaxy & Other Names ${ }^{\mathrm{a}}$ & $\begin{array}{c}D \\
(\mathrm{Mpc})\end{array}$ & References $^{\mathrm{b}}$ & $\begin{array}{c}M_{V} \\
\text { (mag) }\end{array}$ & $\begin{array}{l}R_{D}{ }^{\mathrm{c}} \\
(\mathrm{kpc})\end{array}$ & $\begin{array}{c}\log \mathrm{SFR}_{D}^{\mathrm{H} \alpha \mathrm{d}} \\
\left(M_{\odot} \mathrm{yr}^{-1} \mathrm{kpc}^{-2}\right)\end{array}$ & $\begin{array}{c}\log \mathrm{SFR}_{D}^{\mathrm{FUV} \mathrm{d}} \\
\left(M_{\odot} \mathrm{yr}^{-1} \mathrm{kpc}^{-2}\right)\end{array}$ & $E(B-V)_{f}^{\mathrm{e}}$ \\
\hline DDO 50 & UGC 4305, Holmberg II & 3.05 & 1 & -16.4 & $0.99 \pm 0.05$ & $-1.67 \pm 0.01$ & $-1.55 \pm 0.01$ & 0.028 \\
\hline DDO 53 & UGC 4459 & 3.66 & 2 & -13.9 & $0.73 \pm 0.06$ & $-2.42 \pm 0.01$ & $-2.41 \pm 0.01$ & 0.034 \\
\hline DDO 63 & UGC 5139, Holmberg I & 3.98 & 2 & -14.8 & $0.69 \pm 0.01$ & $-2.32 \pm 0.01$ & $-1.95 \pm 0.00$ & 0.045 \\
\hline NGC 3738 & UGC 6565 & 4.90 & 3 & -17.1 & $0.78 \pm 0.01$ & $-1.66 \pm 0.01$ & $-1.53 \pm 0.01$ & 0.009 \\
\hline Haro 29 & UGCA 281 & 5.90 & 4 & -14.7 & $0.30 \pm 0.01$ & $-0.77 \pm 0.01$ & $-1.07 \pm 0.01$ & 0.013 \\
\hline
\end{tabular}

Notes.

${ }^{a}$ Selected alternate identifications obtained from NED.

b Reference for the distance to the galaxy.

${ }^{\mathrm{c}} R_{D}$ is the disk scale length measured from $V$-band images. From Hunter \& Elmegreen (2006) revised to the distance adopted here.

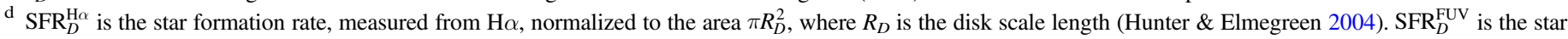
formation rate determined from GALEX FUV fluxes (Hunter et al. 2010, with an update of the GALEX FUV photometry to the GR4/GR5 pipeline reduction).

e Foreground Milky Way extinction from Schlafly \& Finkbeiner (2011).

References. (1) Hoessel et al. 1998; (2) Jacobs et al. 2009; (3) Karachentsev et al. 2003; (4) Schulte-Ladbeck et al. 2001.

(LEGUS; Calzetti et al. 2015) and LITTLE THINGS ${ }^{24}$ (Local Irregulars That Trace Luminosity Extremes, The H I Nearby Galaxy Survey; Hunter et al. 2012). LEGUS is an HST Cycle 21 Treasury survey aimed at exploring star formation from scales of individual stars to kiloparsec-size structures with multiband imaging of 50 galaxies within $16 \mathrm{Mpc}$. The galaxies span the range of star-forming disk galaxies, including dIrrs. The LITTLE THINGS survey is a multiwavelength survey from the far-ultraviolet (FUV) to $21 \mathrm{~cm} \mathrm{H} \mathrm{I} \mathrm{emission} \mathrm{of} 37 \mathrm{dIrr}$ galaxies and 4 BCDs. LITTLE THINGS is aimed at understanding what drives star formation in tiny systems. The LITTLE THINGS galaxies were chosen to be nearby ( $\leqslant 10.3 \mathrm{Mpc}$ ), contain gas so they could form stars, and cover a large range in dwarf galactic properties. The galaxies in common between these two surveys-DDO 50, DDO 53, DDO 63, NGC 3738, and Haro 29-are the focus of this study. Some basic properties of the galaxies are given in Table 1. Haro 29 is classified as a BCD, and NGC 3738 has similar characteristics; both are more extreme in their star-forming properties compared to the other three systems, and NGC 3738 is more extreme than Haro 29.

Table 1 also lists the distances adopted for this study and the references from which they came. These distances are used in order to be consistent with the rest of the LEGUS studies (see Calzetti et al. 2015). We note, however, that recent photometry of stars in the LEGUS galaxies has also yielded distance measurements from the apparent brightness of the tip of the red giant branch (Sabbi et al. 2018). A significant difference exists between the new LEGUS distances and the referenced distances for NGC $3738(5.3 \pm 0.3 \mathrm{Mpc}$ versus $4.9 \mathrm{Mpc})$ and Haro 29 (3.4 $\pm 0.3 \mathrm{Mpc}$ versus 5.9 Mpc). Tully et al. (2013) also give a distance of $5.3 \mathrm{Mpc}$ for NGC 3738, while for Haro 29 they give a distance of $5.7 \mathrm{Mpc}$, which is close to the distance we adopt here. Furthermore, Jacobs et al. (2009) give a distance of $3.42 \mathrm{Mpc}$ for DDO 50, compared to the $3.05 \mathrm{Mpc}$ that we adopt from Hoessel et al. (1998). If these distances were used here instead of those given in Table 1, the masses and brightnesses of objects in NGC 3738 would be $1.2 \times$ higher, in Haro 29 they would be $0.3 \times$ lower, and in DDO 50

\footnotetext{
${ }^{24}$ Funded in part by the National Science Foundation through grants AST0707563, AST-0707426, AST-0707468, and AST-0707835 to US-based LITTLE THINGS team members and with generous technical and logistical support from the National Radio Astronomy Observatory.
}

they would be $2.0 \times$ higher. However, the relative comparison of objects would stay the same.

\subsection{Star Cluster Catalogs}

The sample galaxies were observed with HST's WFC3 imager with filters F275W, F336W, and F438W and the ACS imager with filter F814W. In addition, DDO 50, DDO 53, and DDO 63 were observed with ACS with filter F555W, and NGC 3738 and Haro 29 were observed with ACS with filter F606W. The LEGUS team developed an exhaustive procedure for identifying and checking resolved compact stellar clusters on the images, and details of the catalog preparation are given by Calzetti et al. (2015) and Adamo et al. (2017). The first step is an automatic identification using the algorithm SExtractor (Bertin \& Arnouts 1996). The second step involves the imposition of science-driven criteria aimed at reducing false detections and a visual inspection of selected clusters. This step imposes an absolute magnitude limit, and therefore the compact cluster sample misses low-mass objects, especially at older ages. Cluster catalogs for the LEGUS dwarf galaxies are presented by D. O. Cook et al. (2018a, in preparation).

Photometry of the clusters was performed in all available passbands. The clusters were characterized by their concentration index $(\mathrm{CI})$, the integrated light within the central 1 pixel relative to that within a 3 pixel radius (pixel scale is 0."0396). Aperture corrections, as a function of filter, were made to the cluster photometry using two different methods: (1) taking an average of measurements of isolated clusters over an image and (2) as a function of the CI of the cluster. Here we used both catalogs in the beginning, but we found that it made little difference to the results and subsequently adopted the CI-based aperture corrections. Differences resulting from the two types of aperture corrections are discussed by D. O. Cook et al. (2018a, in preparation). The photometry was corrected for Galactic extinction using Schlafly \& Finkbeiner (2011) with the $E(B-V)$ listed in Table 1. Spectral energy distribution (SED) fits were performed for those clusters with photometry in at least four filters in order to determine the age, mass, and reddening of the cluster within the host galaxy. The fit for one cluster from each galaxy is shown as an example in Figure 1. Several internal reddening curves were used, and here we adopted the catalogs in which the photometry was fit for internal extinction using the curve of Calzetti et al. (2000). The 

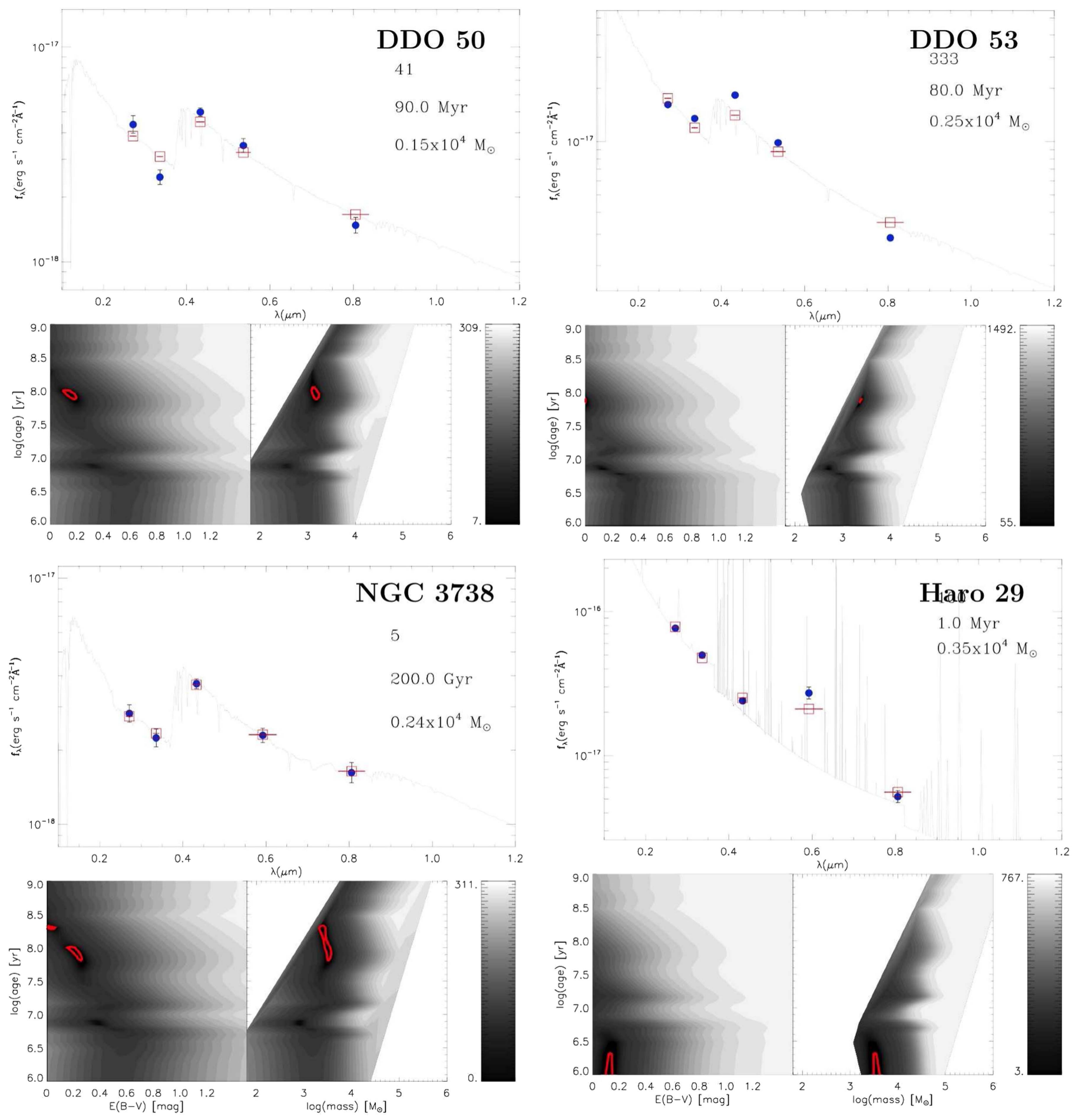

Figure 1. Example SED fits for star clusters: cluster 41 in DDO 50, cluster 333 in DDO 53, cluster 5 in NGC 3738, and cluster 100 in Haro 29. DDO 63 has no clusters. The process is described in detail in Adamo et al. (2017). The top panel shows the flux vs. wavelength: observed fluxes as red squares, and the best-fit spectrum as the solid line and blue filled circles. The age and mass are shown in the upper panel for each cluster. The two panels below the SED panel shows the $\chi^{2}$ distribution in age, mass, and color excess $E(B-V)$. The scale is given to the right of the panels. The red contours are the $68 \%$ confidence level regions.

SED fitting used two methods: (1) the Yggdrasil single stellar population models (Zackrisson et al. 2011), and (2) the stochastically sampled cluster evolutionary models of Krumholz et al. (2015). A Kroupa (2001) stellar initial mass function (IMF) from 0.1 to $120 M_{\odot}$ was assumed. The spread between stochastically based and deterministic derived cluster properties are within the age and mass uncertainties (D. O. Cook et al. 2018a, in preparation), but the differences are particularly noticeable for clusters with masses below $1000 M_{\odot}$ (Krumholz et al. 2015). More details on the production of the cluster catalogs are given by Adamo et al. (2017).

In using these catalogs, we eliminated clusters with masses less than $1000 M_{\odot}$ in order to ensure completeness, those observed in fewer than four filters (class 0), and those having a classification indicating that it is likely a foreground or background source, single star, or artifact (class 4). We 


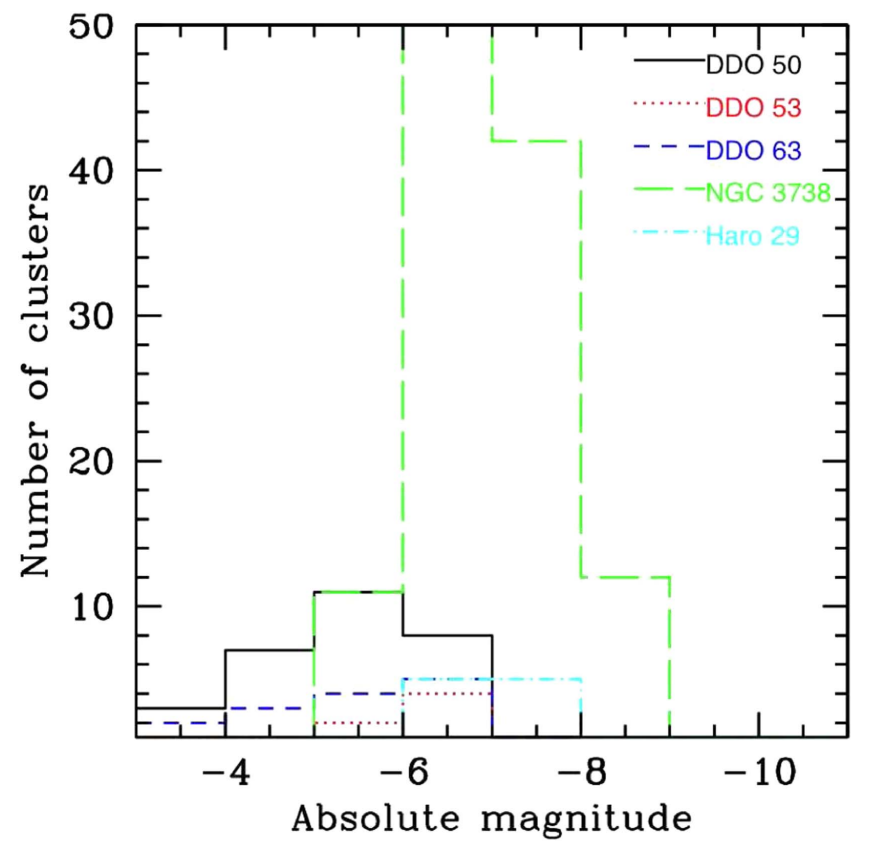

Figure 2. Luminosity function for the clusters in our sample of galaxies. The absolute magnitude is in the F555W filter for DDO 50, DDO 53, and DDO 63 and in the F606W filter for NGC 3738 and Haro 29. NGC 3738 has 79 clusters in the first bin, but we have cropped the $y$-axis at 50 in order to enable the other galaxy luminosity functions to be visible.

included cluster classes 1, 2, and 3, where classes 1 and 2 are compact clusters and class 3 is more likely compact stellar associations (Grasha et al. 2015, 2017; Adamo et al. 2017). We also imposed an age cutoff. We carried age cutoffs of 10, 50, and 100 Myr through the analysis, but differences were small, so we present the results for $100 \mathrm{Myr}$ below, except in the first comparison of cluster properties against environmental properties, where we will also show the result for $10 \mathrm{Myr}$. An age of $100 \mathrm{Myr}$ minimizes losses due to dissolution of clusters (Lamers 2009; Baumgardt et al. 2013; but see Chandar et al. 2017). without decimating the statistics of what are very small cluster samples.

The clusters found automatically were visually inspected in each galaxy down to a cluster absolute magnitude $M_{\mathrm{F} 555 \mathrm{~W}}$ of -6 , or for NGC 3738 and Haro 29 an $M_{\text {F606W }}$ of -6 . We take the peak of the luminosity function of the clusters in a given galaxy as the $90 \%$ cluster completeness limit. In all but one LEGUS galaxy, the peak of the luminosity function is fainter than the limit for visual inspection of the clusters, so a cluster absolute magnitude limit of -6 is a conservative indication of completeness. In Figure 2 we show the luminosity functions for the clusters in our galaxies, and in Figure 3 we plot age versus mass for the clusters in our five galaxies before applying the mass and age cuts that we use in the analysis. The cluster absolute magnitude limit of -6 is translated here into age and mass and shown as a slanting dashed line from young, lowmass clusters up to old, high-mass clusters. We see that our -6 absolute magnitude limit shows potential incompleteness of clusters at the low-mass, older-age corner of our selection box represented by our cutoffs in mass and age. Thus, in the analysis that follows, one should keep in mind that the clusters with masses $<2000 M_{\odot}$ and ages $>35$ Myr may be somewhat more incomplete in this galaxy sample.

The numbers of clusters are given in Table 2, including the number of clusters before the cutoff for mass is applied and the number of clusters with ages $\leqslant 10 \mathrm{Myr}$. There are relatively few clusters in DDO 50, DDO 53, and DDO 63 and even fewer with very young ages. In fact, DDO 63 has no clusters with masses $\geqslant 1000 M_{\odot}$, although it has 12 clusters with smaller masses. DDO 63 does contain O stars.

\subsection{O Star Catalogs}

We also used LEGUS catalogs of candidate O stars in the dwarf galaxies (J. C. Lee et al. 2018, in preparation). These stars were selected to have magnitudes in NUV, $U, B$, and $V$ passbands; have been flagged in the original stellar catalogs as having a point-source profile; have an accurate $\mathrm{F} 275 \mathrm{~W}$ magnitude brighter than 25.5 (corresponding to a $3 \sigma$ detection); and have a reddening-free $Q$ value greater than 1.6 with an uncertainty less than 0.075. $Q$ is defined by $Q=$ $\left(M_{F 275 W}-M_{U}\right)-K\left(M_{U}-M_{B}\right)$, where $K$ is a constant that is computed using $E(\mathrm{NUV}-U) / E(U-B)$ with a Milky Way dust type $\left(R_{V}=3.1\right)$. Such a value of $Q$ selects for $\mathrm{O}$ stars, in particular stars with masses $\geqslant 17 M_{\odot}$. Lower-mass stars, having redder colors, have $Q$ values lower than this cutoff. However, O stars within H II regions could be missed owing to higher differential extinction. Details on the LEGUS stellar photometry can be found in Sabbi et al. (2018).

The magnitude cutoff of $25.5 \mathrm{mag}$ in $\mathrm{F} 275 \mathrm{~W}$ is near the magnitude at which incompleteness starts to become severe in these nearby galaxies. However, in practice the faintest F275W magnitudes in the catalogs are significantly brighter than this: 23.5, 22.6, 23.2, 22.7, and 22.5 in DDO 50, DDO 53, DDO 63, NGC 3738, and Haro 29, respectively. Thus, the populations of stars we are working with are at least 2 mag brighter than the typical limiting magnitude of 25.5 in F275W. Hence, we are not likely to be significantly affected by incompleteness issues that might also bias the sample toward more massive stars in more distant galaxies.

The photometry is only corrected for foreground extinction according to Schlafly \& Finkbeiner (2011). In our analysis where we use the F275W magnitudes of the stars, we apply an additional correction for internal extinction using an $E(B-V)=0.05$ mag with the attenuation curve of Calzetti et al. (2000). Actual extinctions are not known for our sample of galaxies, and so $0.05 \mathrm{mag}$ is used as a typical extinction for stars not buried in $\mathrm{H}$ II regions. This value is half of the average $E(B-V)$ derived from $\mathrm{H}$ II region Balmer decrements for typical dwarfs (Hunter \& Elmegreen 2006), but $E(B-V)$ could be larger in some dwarfs (Cignoni et al. 2018). The numbers of candidate $\mathrm{O}$ stars are given in Table 2 .

When we discuss the properties of star clusters and $\mathrm{O}$ stars below, one issue will be our ability to distinguish compact clusters from stars. Our galaxy sample is relatively nearby, and dIrr galaxies have lower stellar densities than spirals, but we have approached this distinction carefully and systematically for the LEGUS sample as a whole. The process is described in detail by Adamo et al. (2017), but the key step in distinguishing clusters from stars is summarized here. The CI is determined for a "training sample" of objects that are clearly stars and those that are clearly clusters, and a histogram of the CI is plotted. There is a clear gap between the CI of stars and the CI of compact clusters, and this is used to determine the CI cut for clusters and stars. Figure 4 shows a plot of the CI for all sources extracted from our sample of galaxies, and the red vertical line indicates the CI value used to distinguish compact clusters from stars in each galaxy. One can see that even in the more distant 
Table 2

Numbers of Clusters, OB Associations, and O Star Candidates

\begin{tabular}{|c|c|c|c|c|c|}
\hline \multirow[b]{2}{*}{ Galaxy } & \multicolumn{3}{|c|}{ No. Clusters } & \multirow[b]{2}{*}{ No. OB Assoc } & \multirow[b]{2}{*}{ No. O Stars } \\
\hline & Masses $>10^{3} M_{\odot}$, Ages $\leqslant 10 \mathrm{Myr}$ & Masses $>10^{3} M_{\odot}$, Ages $\leqslant 100 \mathrm{Myr}$ & No Mass Cut, Ages $\leqslant 100 \mathrm{Myr}$ & & \\
\hline DDO 50 & 7 & 11 & 56 & 17 & 404 \\
\hline DDO 53 & 1 & 1 & 7 & 11 & 101 \\
\hline DDO 63 & 0 & 0 & 12 & 6 & 105 \\
\hline NGC 3738 & 51 & 138 & 172 & 3 & 281 \\
\hline Haro 29 & 8 & 9 & 13 & 7 & 61 \\
\hline
\end{tabular}

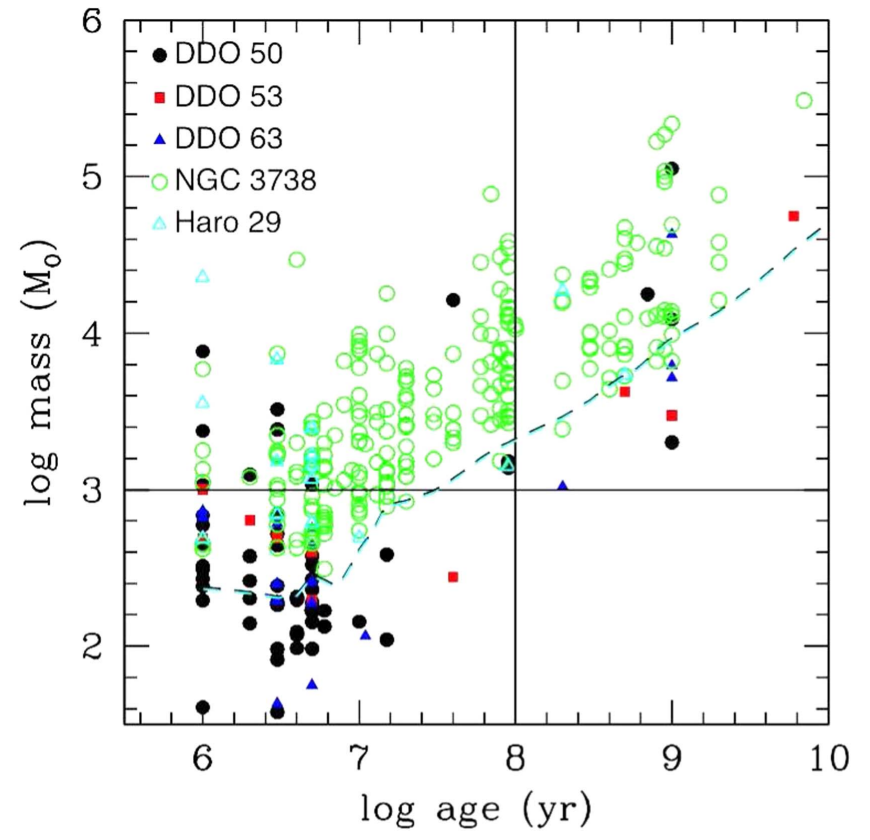

Figure 3. Mass vs. age for the star clusters after cutting clusters from the catalogs on the basis of class ( 0 and 4$)$ and number of filters with observations ( $<4$ filters). The vertical solid black line shows the age cut we apply throughout the analysis (age $>100 \mathrm{Myr}$ ), and the horizontal solid black line marks the cluster mass cut (mass $<1000 M_{\odot}$ ). The slanted dashed line marks the catalog limits for visual inspection of the clusters defined as $M_{\mathrm{F} 555 \mathrm{~W}}=-6$ for DDO 50, DDO 53, and DDO 63 and $M_{\mathrm{F} 606 \mathrm{~W}}=-6$ for NGC 3738 and Haro 29, a conservative estimate of completeness limit. The concern for incompleteness is at the low-mass $\left(<2000 M_{\odot}\right)$, older-age ( $>35$ Myr) corner of our selection box.

galaxies in our sample (NGC 3738 and Haro 29) there is a fairly clear drop to higher $\mathrm{CI}$ index. One can also see extended tails to higher CI values in each galaxy because the clusters have a broader distribution of CI values than stars.

\subsection{OB Associations}

Not all star-forming units are clusters or compact associations, and there are larger groups of $\mathrm{O}$ stars in all five galaxies. Guided by the distribution of $\mathrm{O}$ stars and emission in the F275W images, we have, by eye, outlined apparent OB associations. These are objects that appear as obvious density enhancements in the number of $\mathrm{O}$ stars per area. The size of an $\mathrm{OB}$ association is taken to be the radius of the circle that encompasses the $\mathrm{O}$ stars and F275W emission.

The northwestern part of NGC 3738 is problematic in regard to $\mathrm{OB}$ association identification; there are so many $\mathrm{O}$ stars and clumps that it was difficult to decide whether it was a very large single association or a close grouping of many individual clumps. Whichever way it is described, it is an extraordinary region in terms of its size and density of $\mathrm{O}$ stars (and compact clusters), and here we chose to emphasize that by considering it as a single region with a radius of $260 \mathrm{pc}$. In addition, most of the star formation in NGC 3738 is concentrated to this region, and we discuss the morphology of star formation in NGC 3738 and a similar galaxy DDO 187 in Hunter et al. (2018). If we had described that region instead as many smaller associations, what would we have found? It is likely that most of the smaller associations in the center of the region, while having smaller radii and stellar masses, would have similar stellar mass densities and be associated with similar galactic environmental properties (see Figure 11 below). However, some of the smaller associations along the edges of the region in this scenario would have lower mass densities and be described as having less extreme environmental properties as well. It is unlikely that taking this alternate definition of the region in NGC 3738 would alter the results of the analysis presented here.

We measured photometry in the circles encompassing the $\mathrm{OB}$ associations, chosen by eye to include the $\mathrm{O}$ stars and $\mathrm{F} 275 \mathrm{~W}$ emission, on the HST images. We subtracted any clusters within the boundary of the OB association and subtracted background measured in an annulus around the association. The photometry is on the Vega system for an infinite aperture size, and it was corrected for foreground extinction $E(B-V)_{f}$ according to Schlafly \& Finkbeiner (2011). We performed SED fitting to the photometry, using the Yggdrasil single stellar population models as for the star clusters, to determine the mass, age, and $E(B-V)_{i}$ internal to the host galaxy. Two examples of the SED fitting are shown in Figure 5. The OB associations are identified in Table 3, and the mass, the mass divided by the area of the encompassing circle, the age, and $E(B-V)_{i}$ are given there. For absolute F275W magnitudes, we applied the additional extinction correction for internal reddening, as listed in Table 3, using an $A_{F 275 W} / E(B-V)$ of 7.43 (Calzetti et al. 2000). The goodness of the fit (probability that the chi-square exceeds a particular value $\chi^{2}$ by chance; Press et al. 2007) is also given in Table 3, where a value of 1 denotes a good fit and a value near 0 means that the fit is not well constrained. Uncertainties in age and mass come from the maximum values and minimum values allowed by the fits. The ages of the $\mathrm{OB}$ associations range from 1 to $50 \mathrm{Myr}$, and radii of the encompassing circles range from 20 to $300 \mathrm{pc}$.

\subsection{LEGUS Star Formation Rate Maps}

We also make use of SFR surface density maps produced by D. Thilker et al. (2018, in preparation). The maps we used were made at $0.25 \mathrm{kpc}$ resolution and use GALEX FUV images to determine the SFR. Wide-field Infrared Survey Explorer 

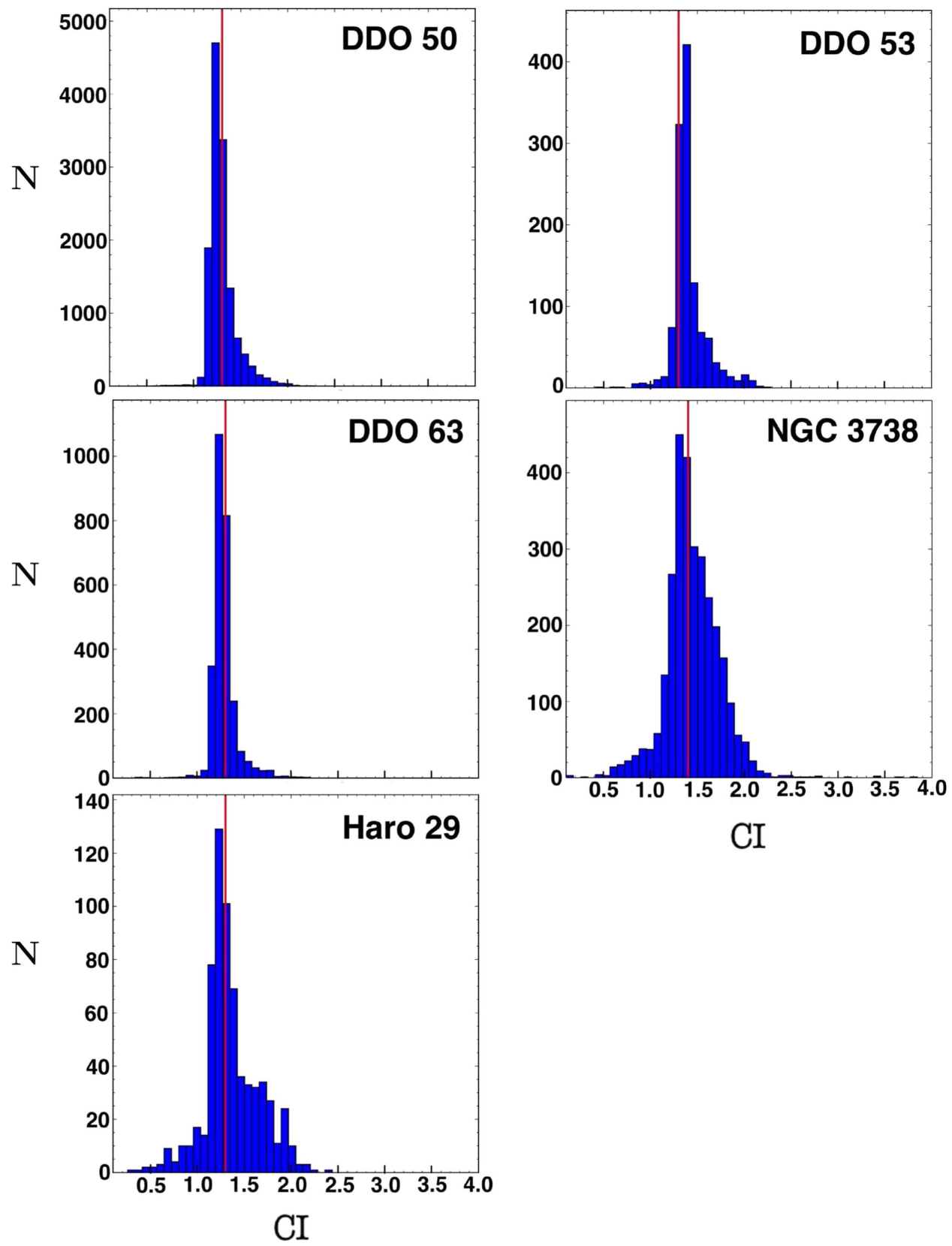

Figure 4. Number of stars and clusters as a function of CI for all sources identified in each galaxy. The vertical red line denotes the CI marking the boundary between stars (lower CI) and clusters (higher CI) determined from a training sample of unambiguous stars and clusters. See Adamo et al. (2017) for more details on the process of distinguishing stars from clusters used in the LEGUS sample.

(WISE) HiRes W4 $22 \mu \mathrm{m}$ maps, if the galaxy was detected, were used to correct for extinction. For DDO 53 the "unWISE" images (unofficial, unblurred co-adds of the WISE images) were used instead (Lang 2014; Meisner et al. 2017). We followed the method of T. H. Jarrett (2018, private communication; see also Jarrett et al. 2012) and the prescription by Boquien et al. (2016), attempting to allow for the spatially variable contribution of old stellar populations to the dust heating, with the scaling as a function of local FUV-W1 color. This method scales the IR bands by a factor of 6.0, appropriate for local scales. If instead we used a scaling appropriate for global measurements of galaxies, such as the value of 3.89 determined by Hao et al. (2011), we find that the SFR density in spots in the centers of the galaxies would be lower by as much as a factor of two.
The FUV was corrected for foreground extinction following Peek \& Schiminovich (2013). The maps use a Kroupa IMF from 0.1 to $100 M_{\odot}$ with an SFR timescale $\geqslant 100 \mathrm{Myr}$ (Kroupa 2001). The units of the maps are $M_{\odot} \mathrm{yr}^{-1} \mathrm{kpc}^{-2}$. We note, however, that SFRs determined over regions, especially small regions, within dwarf galaxies can be highly affected by the stochastic sampling of a universal IMF and time-dependent fluctuations in the SFR (Fumagalli et al. 2011; da Silva et al. 2014).

We have integrated the SFR in DDO 50 over all pressure regions in order to compare to the SFR determined from the GALEX FUV based on a calibration from resolved stellar populations by McQuinn et al. (2015). Their value, converted to our distance, is $\log \mathrm{SFR}=-1.21 \pm 0.17$. This is 1.4 times larger than our value from the SFR map described here, but 

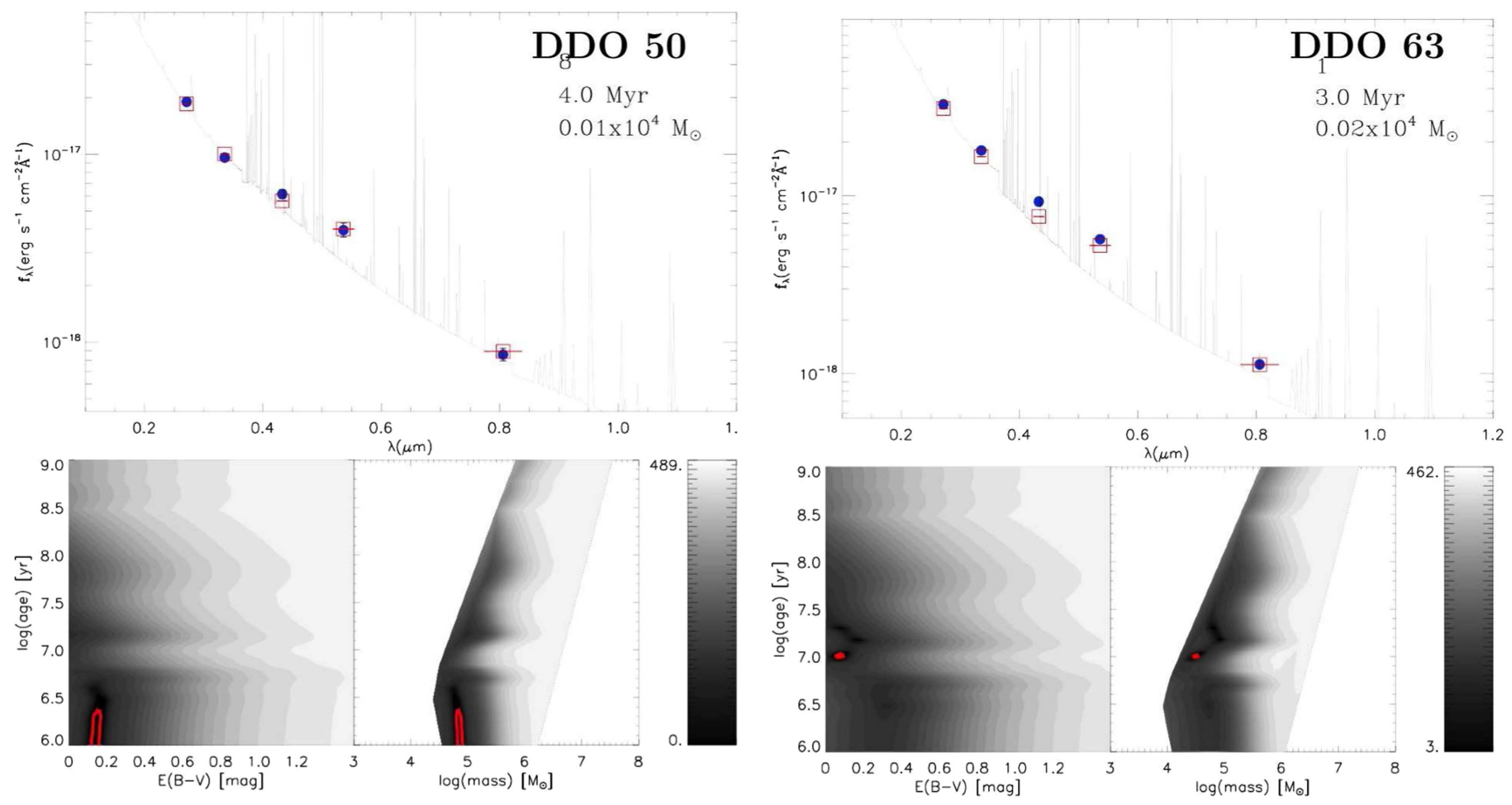

Figure 5. Example SED fits for OB associations: \#8 in DDO 50 and \#1 in DDO 63. The top panel shows the flux vs. wavelength: observed fluxes as red squares and the best-fit spectrum as the solid line and blue filled circles. The age and mass are shown in the upper panel for each cluster. The two panels below the SED panel show the $\chi^{2}$ distribution in age, mass, and color excess $E(B-V)$. The scale is given to the right of the panels. The red contours are the $68 \%$ confidence level regions.

they are the same within $1 \sigma$. The integrated SFR from GALEX FUV given in Table 1 from Hunter et al. (2012) is 50\% lower than the McQuinn et al. (2015) value.

\subsection{Galactic Environments}

The LITTLE THINGS data sets include HI line maps obtained with the Very Large Array (VLA) interferometer. ${ }^{25}$ The H I maps are characterized by high sensitivity $\left(\leqslant 1.1 \mathrm{Jy}^{\text {beam }}{ }^{-1}\right.$ per channel), high spectral resolution $\left(\leqslant 2.6 \mathrm{~km} \mathrm{~s}^{-1}\right)$, and high angular resolution $\left(\sim 6^{\prime \prime}\right)$. To obtain maps of gas surface density, we converted the naturally weighted integrated (moment 0 ) $\mathrm{H} \mathrm{I}$ maps to units of column density (atoms $\mathrm{cm}^{-2}$ ) and multiplied by 1.36 to account for helium. In addition, we use $B$ and $V$ images obtained at Lowell Observatory to determine the stellar mass density in each pixel. We used the $B-V$ color to determine the mass-to-light ratio using a formula determined from SED fitting to the LITTLE THINGS photometry (Herrmann et al. 2016), and with $L_{V}$ we determined the stellar mass in each pixel. (Note that we do not use WISE near-IR images to determine the stellar mass because the dIrrs are not generally detected by WISE. We also do not use Spitzer $3.6 \mu \mathrm{m}$ images for $\mathrm{S} / \mathrm{N}$ issues. See Zhang \& Puzia (2017) for a discussion on the effect of lack of red colors on stellar mass estimates). From Zhang \& Puzia (2017) we can expect that these stellar masses are good to a factor of two, and we adopt an uncertainty of 0.3 dex in the stellar mass surface densities. The stellar mass densities were converted from a Chabrier (2003) stellar IMF to the Kroupa (2001) IMF used in

\footnotetext{
25 The VLA, now the NSF's Karl G. Jansky Very Large Array (JVLA), is a facility of the National Radio Astronomy Observatory. The National Radio Astronomy Observatory is a facility of the National Science Foundation operated under cooperative agreement by Associated Universities, Inc.
}

the cluster and $\mathrm{OB}$ association masses as indicated by Herrmann et al. (2016). The integrated H I maps and stellar mass maps are shown in Figure 6.

The H I and stellar surface density maps were combined at their native resolutions to produce maps of the hydrostatic midplane pressure in each galaxy:

$$
P=2.934 \times 10^{-55} \times \Sigma_{\text {gas }}\left(\Sigma_{\text {gas }}+\left(\sigma_{g} / \sigma_{*}\right) \Sigma_{*}\right)\left[\mathrm{g} /\left(\mathrm{s}^{2} \mathrm{~cm}\right)\right],
$$

where $\Sigma$ is a surface density and $\sigma$ is a velocity dispersion (Elmegreen 1989). Here $\Sigma_{\text {gas }}$ is determined solely from $\mathrm{H} \mathrm{I}+\mathrm{He}$ since the molecular $\mathrm{H}_{2}$ content is unknown. Although the molecular gas is more closely tied to star formation than the atomic gas, the $\mathrm{HI}+\mathrm{He}$ is the material that is available to become molecular on a larger spatial scale. Molecular gas is most likely to be found within the denser H I clouds in lowmetallicity environments, so if molecular content were known and included, it would probably increase the pressure in the higher-pressure regions rather than increasing the pressure in low H I density regions. The gas velocity dispersion was derived from the HI moment 2 maps. The stellar velocity dispersion was estimated using $\log \sigma_{*}=-0.15 M_{B}-1.27$ from Swaters (1999), where $M_{B}$ is the integrated absolute $B$ magnitude of the galaxy. Because dIrrs are gas dominated and since the gas surface density enters as $\Sigma_{\text {gas }}^{2}$, the pressure maps are dominated by the $\mathrm{H}$.

We estimate the uncertainties in $\Sigma_{\mathrm{H} \text { I }}$ in the integrated $\mathrm{HI}$ moment 0 map from the HI data cube channel rms (Hunter et al. 2012) and assume that the number of channels contributing to each pixel in the integrated moment zero map is the typical velocity profile FWHM divided by the channel width, about six channels. We take a typical pressure region of 
Table 3

OB Associations

\begin{tabular}{|c|c|c|c|c|c|c|c|c|c|c|}
\hline Galaxy & ID & $\begin{array}{l}\text { R.A. (2000) } \\
\text { (hh mm ss.s) }\end{array}$ & $\begin{array}{l}\text { Decl. (2000) } \\
\text { (dd mm ss) }\end{array}$ & $\begin{array}{c}R \\
(\mathrm{pc})\end{array}$ & $M_{F 275 W, 0}$ & $\begin{array}{c}\log \text { Mass } \\
\left(M_{\odot}\right)\end{array}$ & $\begin{array}{c}\log \text { Age } \\
\text { (years) }\end{array}$ & $\begin{array}{l}\log \text { Mass/Area } \\
\left(M_{\odot} \mathrm{pc}^{-2}\right)\end{array}$ & $\begin{array}{l}\text { Goodness } \\
\text { of Fit }\end{array}$ & $E(B-V)$ \\
\hline \multirow[t]{12}{*}{ DDO 50} & 1 & 81917.4 & +704342 & 43.4 & $-11.65 \pm 0.002$ & $3.90_{-0.28}^{-.07}$ & $6.48_{-0.48}^{+0.12}$ & 0.128 & 0.8 & $0.22_{-0.07}^{+0.04}$ \\
\hline & 2 & 81929.1 & +70433 & 56.8 & $-12.19 \pm 0.001$ & $4.25_{-0.28}^{-.08}$ & $6.30_{-0.30}^{+0.30}$ & 0.243 & 0.9 & $0.11_{-0.05}^{+0.03}$ \\
\hline & 3 & 81929.1 & +704251 & 53.9 & $-11.76 \pm 0.001$ & $3.97_{-0.30}^{-.10}$ & $6.48_{-0.18}^{+0.12}$ & 0.009 & 0.9 & $0.09_{-0.08}^{+0.03}$ \\
\hline & 4 & 81930.2 & +704241 & 65.1 & $-11.54 \pm 0.001$ & $4.05_{-0.28}^{-.10}$ & $6.00_{-0.00}^{+0.60}$ & -0.074 & 0.8 & $0.07_{-0.04}^{+0.04}$ \\
\hline & 5 & 81926.5 & +704248 & 60.4 & $-11.42 \pm 0.002$ & $3.82_{-0.26}^{-.08}$ & $6.48_{-0.18}^{+0.12}$ & -0.239 & 0.9 & $0.14_{-0.06}^{+0.04}$ \\
\hline & 8 & 81912.2 & +70438 & 94.9 & $-13.58 \pm 0.001$ & $4.86_{-0.25}^{-.08}$ & $6.00_{-0.00}^{+0.30}$ & 0.408 & 1.0 & $0.11_{-0.03}^{+0.05}$ \\
\hline & 9 & 81927.4 & +704158 & 59.8 & $-12.34 \pm 0.001$ & $4.34_{-0.25}^{-.10}$ & $6.00_{-0.00}^{+0.30}$ & 0.290 & 0.9 & $0.14_{-0.04}^{+0.05}$ \\
\hline & 10 & 81925.9 & +704153 & 59.2 & $-10.89 \pm 0.002$ & $3.73_{-0.18}^{-.08}$ & $6.70_{-0.00}^{+0.00}$ & -0.312 & 0.8 & $0.02_{-0.02}^{+0.03}$ \\
\hline & 11 & 81923.5 & +704153 & 93.8 & $-11.25 \pm 0.001$ & $4.39_{-0.19}^{-.03}$ & $7.18_{-0.03}^{+0.00}$ & -0.051 & 0.4 & $0.03_{-0.03}^{+0.04}$ \\
\hline & 12 & 81923.0 & +70423 & 64.5 & $-9.66 \pm 0.003$ & $3.41_{-0.13}^{+0.36}$ & $6.85_{-0.24}^{+0.27}$ & -0.706 & 0.5 & $0.00_{-0.00}^{+0.23}$ \\
\hline & 13 & 81923.1 & +704258 & 90.3 & $-12.02 \pm 0.002$ & $4.02_{-0.25}^{+0.18}$ & $6.48_{-0.00}^{+0.70}$ & -0.388 & 0.1 & $0.16_{-0.16}^{+0.04}$ \\
\hline & 17 & 81923.8 & +70428 & 34.6 & $-9.64 \pm 0.003$ & $3.22_{-0.30}^{-.10}$ & $6.30_{-0.30}^{+0.30}$ & -0.355 & 0.9 & $0.05_{-0.04}^{+0.06}$ \\
\hline \multirow[t]{11}{*}{ DDO 53} & 1 & 8346.9 & +661056 & 86.5 & $-12.61 \pm 0.001$ & $4.21_{-0.26}^{-.08}$ & $6.48_{-0.18}^{+0.12}$ & -0.161 & 1.0 & $0.13_{-0.06}^{+0.04}$ \\
\hline & 2 & 8349.8 & +661044 & 42.9 & $-9.44 \pm 0.004$ & $3.55_{-0.15}^{-.07}$ & $7.18_{-0.03}^{+0.00}$ & -0.212 & 0.6 & $0.00_{-0.00}^{+0.02}$ \\
\hline & 3 & 8347.8 & +661051 & 37.3 & $-10.39 \pm 0.003$ & $3.50_{-0.26}^{-.10}$ & $6.00_{-0.00}^{+0.60}$ & -0.140 & 0.9 & $0.10_{-0.04}^{+0.05}$ \\
\hline & 4 & 8348.7 & +661052 & 19.7 & $-9.70 \pm 0.003$ & $3.17_{-0.28}^{-.08}$ & $6.30_{-0.30}^{+0.30}$ & 0.084 & 0.9 & $0.03_{-0.03}^{+0.05}$ \\
\hline & 5 & 8348.4 & +661049 & 22.5 & $-9.74 \pm 0.004$ & $3.07_{-0.26}^{-.08}$ & $6.48_{-0.00}^{+0.12}$ & -0.132 & 1.0 & $0.08_{-0.06}^{+0.04}$ \\
\hline & 6 & 8348.7 & +661038 & 30.2 & $-8.69 \pm 0.006$ & $2.72_{-0.27}^{-.06}$ & $6.70_{-0.22}^{+0.00}$ & -0.738 & 0.2 & $0.01_{-0.01}^{+0.04}$ \\
\hline & 7 & 8343.8 & +661037 & 31.6 & $-10.03 \pm 0.003$ & $3.28_{-0.25}^{-.06}$ & $6.70_{-0.22}^{+0.00}$ & -0.218 & 0.6 & $0.07_{-0.04}^{+0.04}$ \\
\hline & 8 & 8348.6 & +661047 & 23.2 & $-7.42 \pm 0.011$ & $2.74_{-0.15}^{-.03}$ & $7.18_{-0.03}^{+0.00}$ & -0.489 & 0.3 & $0.00_{-0.00}^{+0.04}$ \\
\hline & 9 & 8343.4 & +661041 & 42.2 & $-10.52 \pm 0.003$ & $3.47_{-0.30}^{+0.16}$ & $6.70_{-0.22}^{+0.48}$ & -0.278 & 0.5 & $0.11_{-0.11}^{+0.04}$ \\
\hline & 10 & 8349.7 & +661038 & 33.8 & $-10.50 \pm 0.004$ & $3.77_{-0.22}^{-.01}$ & $7.00_{-0.00}^{+0.04}$ & 0.216 & 0.1 & $0.18_{-0.05}^{+0.04}$ \\
\hline & 11 & 8346.0 & +661021 & 33.8 & $-9.34 \pm 0.004$ & $3.01_{-0.21}^{-.06}$ & $6.70_{-0.00}^{+0.00}$ & -0.544 & 0.8 & $0.03_{-0.03}^{+0.04}$ \\
\hline & 2 & 113548.4 & +543118 & 138.4 & $-13.77 \pm 0.001$ & $6.12_{-0.71}^{+0.06}$ & $7.70_{-0.92}^{+0.30}$ & 1.341 & 1.0 & $0.05_{--.11}^{+0.36}$ \\
\hline & 3 & 113548.8 & +543126 & 66.8 & $-12.48 \pm 0.001$ & $5.65_{-0.71}^{+0.07}$ & $7.70_{-0.92}^{+0.30}$ & 1.503 & 1.0 & $0.04_{--.12}^{+0.37}$ \\
\hline \multirow[t]{7}{*}{ Haro 29} & 1 & 122615.7 & +482938 & 56.7 & $-14.51 \pm 0.001$ & $5.13_{-0.25}^{-.10}$ & $6.00_{-0.00}^{+0.30}$ & 1.126 & 0.3 & $0.12_{-0.04}^{+0.03}$ \\
\hline & 2 & 122616.0 & +482937 & 45.3 & $-13.49 \pm 0.001$ & $4.76_{-0.23}^{-.10}$ & $6.00_{-0.00}^{+0.30}$ & 0.950 & 0.0 & $0.16_{-0.03}^{+0.03}$ \\
\hline & 3 & 122616.5 & +482937 & 45.3 & $-12.49 \pm 0.003$ & $4.33_{-0.25}^{-.10}$ & $6.00_{-0.00}^{+0.30}$ & 0.520 & 0.8 & $0.18_{-0.03}^{+0.04}$ \\
\hline & 4 & 122616.3 & +482940 & 57.8 & $-12.57 \pm 0.002$ & $4.37_{-0.25}^{-.10}$ & $6.00_{-0.00}^{+0.30}$ & 0.349 & 0.6 & $0.16_{-0.04}^{+0.04}$ \\
\hline & 5 & 122616.2 & +482935 & 36.3 & $-12.11 \pm 0.004$ & $4.19_{-0.25}^{-.11}$ & $6.00_{-0.00}^{+0.30}$ & 0.574 & 0.1 & $0.24_{-0.04}^{+0.03}$ \\
\hline & 6 & 122617.2 & +482939 & 54.4 & $-12.48 \pm 0.002$ & $4.20_{-0.22}^{-.05}$ & $6.60_{-0.12}^{+0.00}$ & 0.231 & 0.7 & $0.14_{-0.03}^{+0.07}$ \\
\hline & 7 & 122616.9 & +482938 & 23.8 & $-10.81 \pm 0.007$ & $3.54_{-0.24}^{-.05}$ & $6.60_{-0.30}^{+0.00}$ & 0.289 & 0.9 & $0.24_{-0.04}^{+0.07}$ \\
\hline
\end{tabular}

radius $200 \mathrm{pc}$ and determine the number of H I pixels $N$ summed in such a region for the galaxy's distance, and the uncertainty in $\Sigma_{\mathrm{H} \text { I }}$ goes as $\sqrt{N}$. The uncertainty in $\log \Sigma_{\mathrm{HI}}$ is 0.02 (units $M_{\odot} \mathrm{pc}^{-2}$ ) for Haro 29, 0.04 for NGC 3738, 0.07 for DDO 63, 0.06 for DDO 53, and 0.09 for DDO 50. For a typical $\mathrm{HI}$ surface density of $10 M_{\odot} \mathrm{pc}^{-2}$, the uncertainty in $\Sigma_{\mathrm{HI}}$ is from 5\% for Haro 29 up to $20 \%$ for DDO 50 . The uncertainty in the pressure is determined from the fact that the pressure goes as $\Sigma_{\mathrm{H} \text { I }}^{2}$, so the uncertainty of $\log P$ is $2 \times$ the uncertainty of $\log \Sigma_{\mathrm{H} \text { I }}$.
For an idea of what to expect related to pressure, Figure 2 of Elmegreen \& Parravano (1994) shows the minimum pressure expected for star formation to take place as a function of metallicity and stellar radiation field. This predicts that most dIrr galaxies have pressures at or below the minimum. In the sections that follow we divide the observed pressures into bins with bin 1 pressures below $4 \times 10^{-13} \mathrm{~g} \mathrm{~s}^{-2} \mathrm{~cm}^{-1}$, bin 2 pressures up to $4 \times 10^{-12} \mathrm{~g} \mathrm{~s}^{-2} \mathrm{~cm}^{-1}$, and bin 3 pressures above that. For context, the typical total midplane pressure in the solar neighborhood is of order $3 \times 10^{-12} \mathrm{~g} \mathrm{~s}^{-2} \mathrm{~cm}^{-1}$, near 


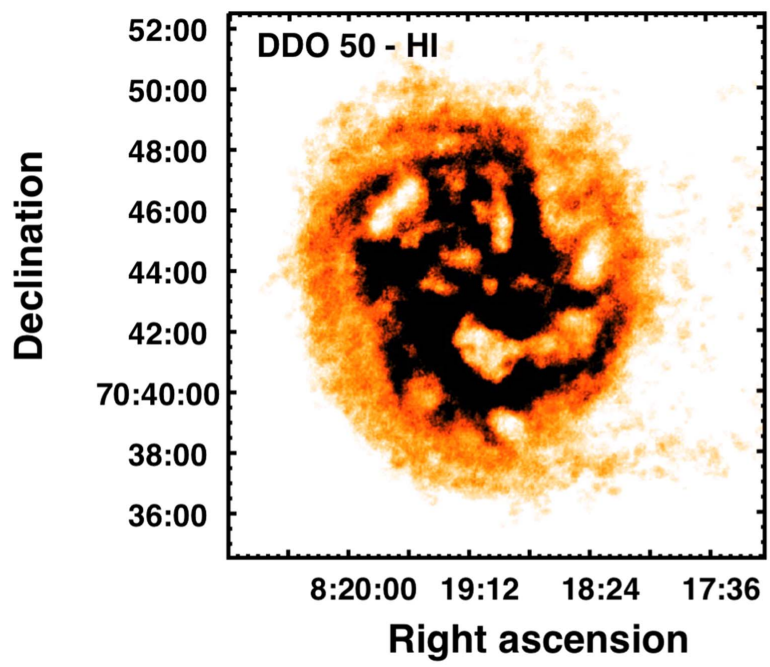

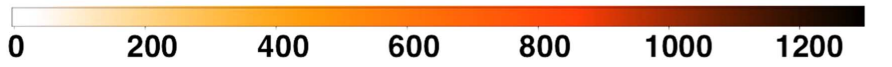
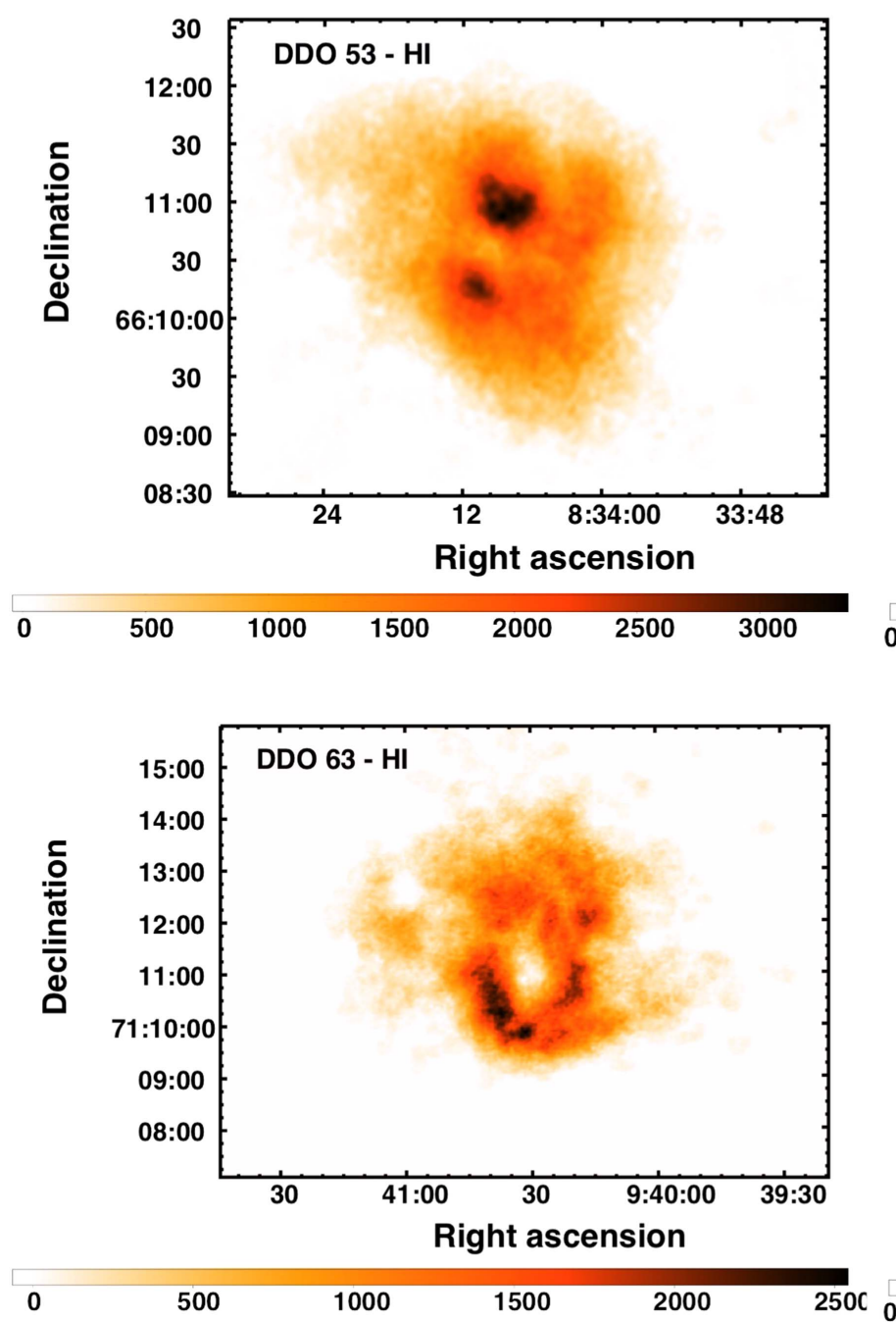

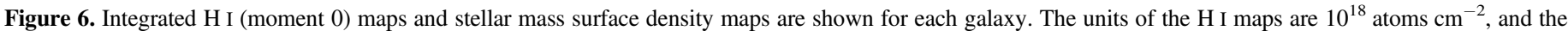
units of the stellar mass surface density maps are $M_{\odot} \mathrm{pc}^{-2}$.

the boundary between bins 2 and 3 (Cox 2005). The pressure in typical H II regions in dIrrs is also relatively high and would fall between bins 2 and 3, but the typical disk of a dIrr is lower
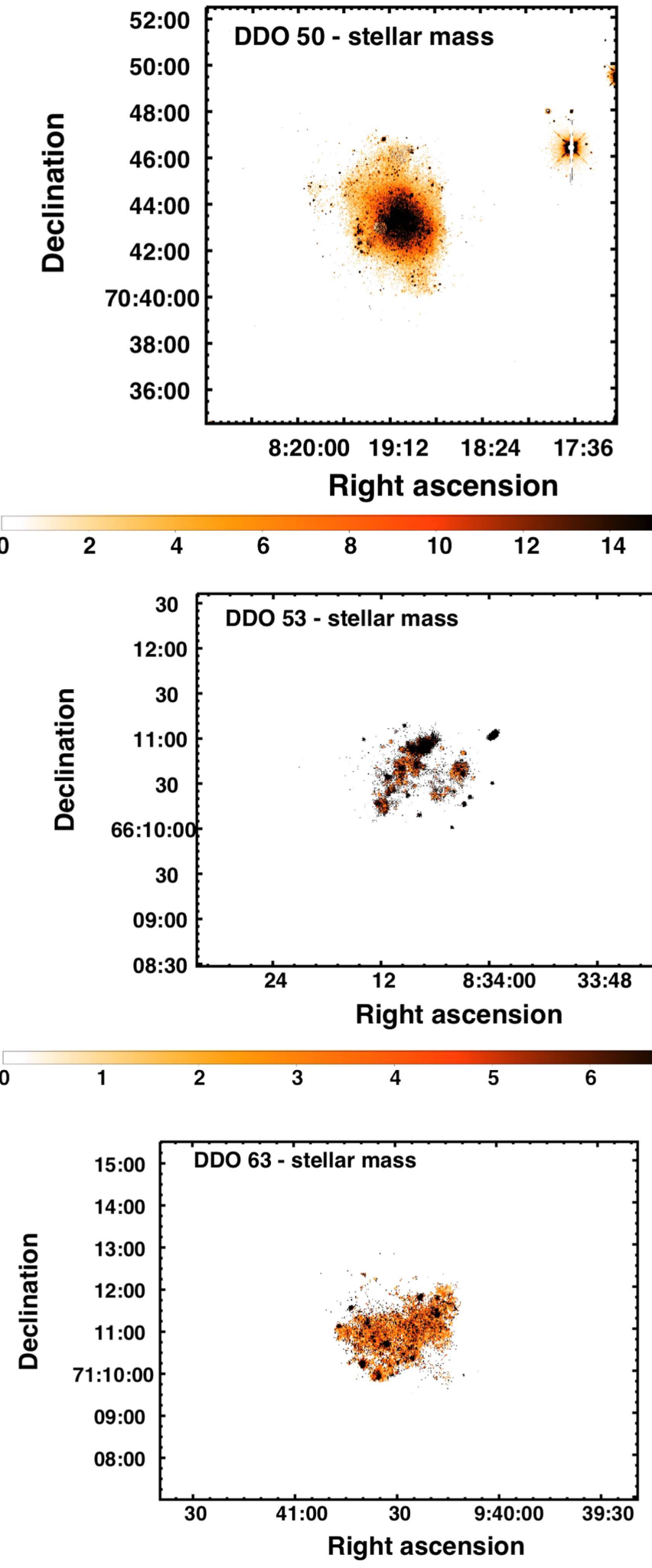

\begin{tabular}{llllllll|}
\hline 0 & 2 & 4 & 6 & 8 & 10 & 12 & 14
\end{tabular}
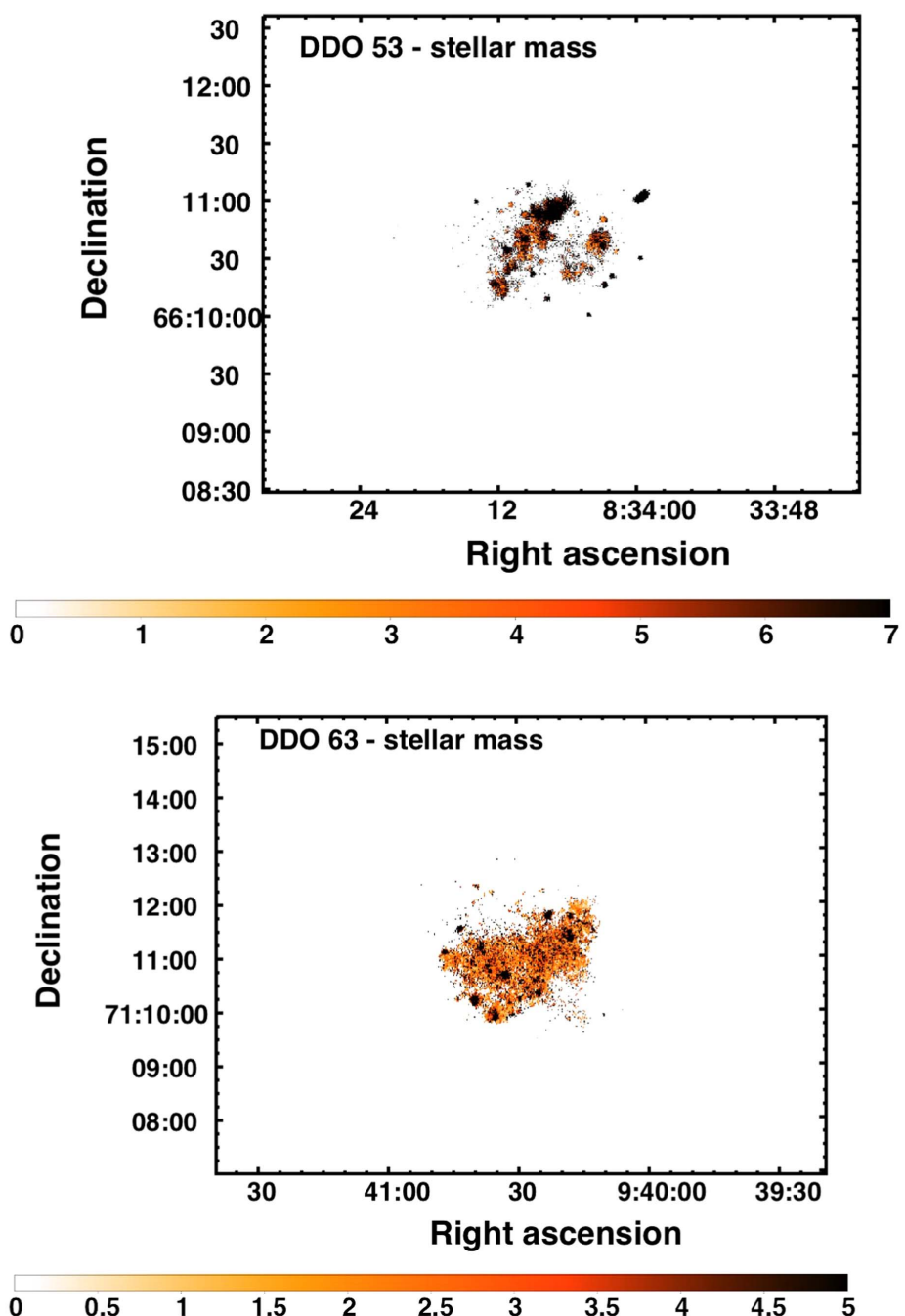

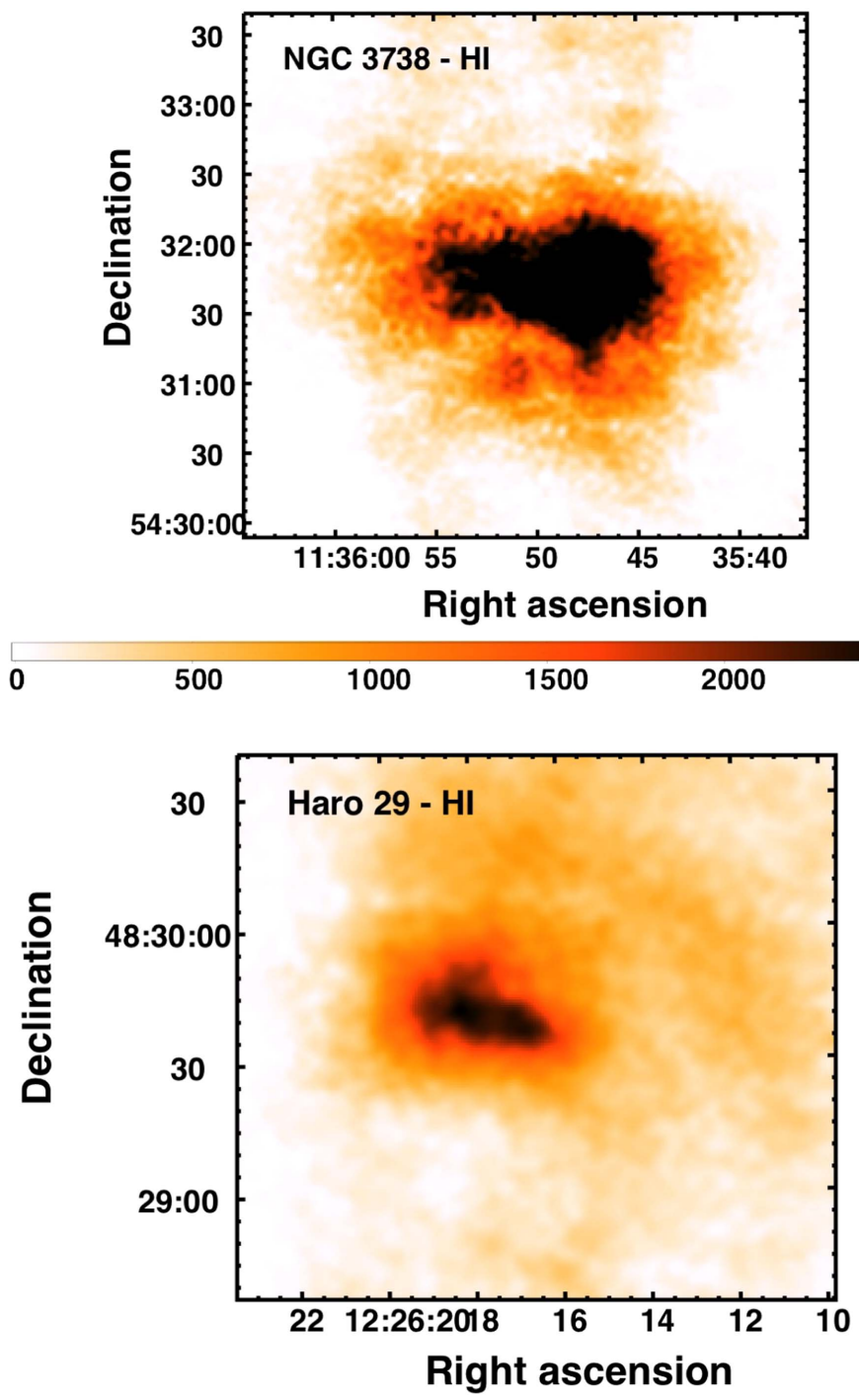

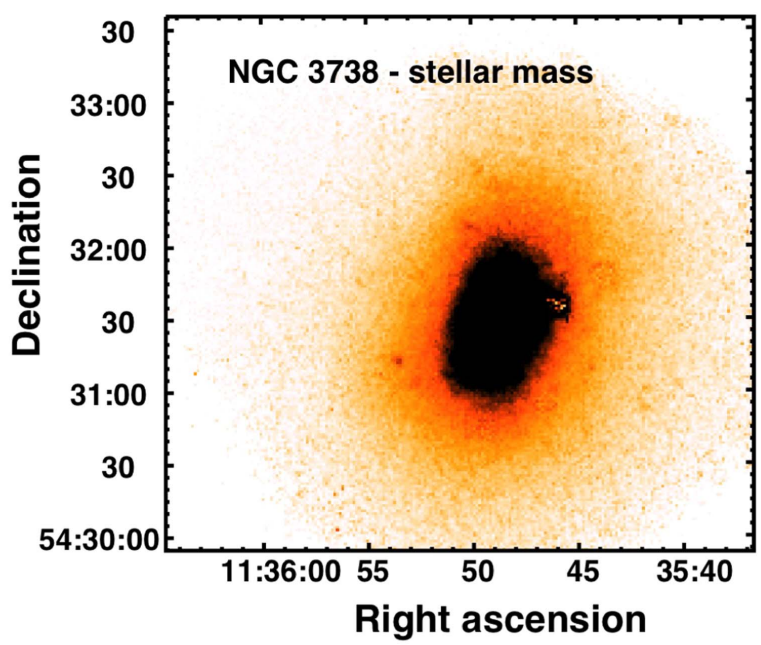

0

10

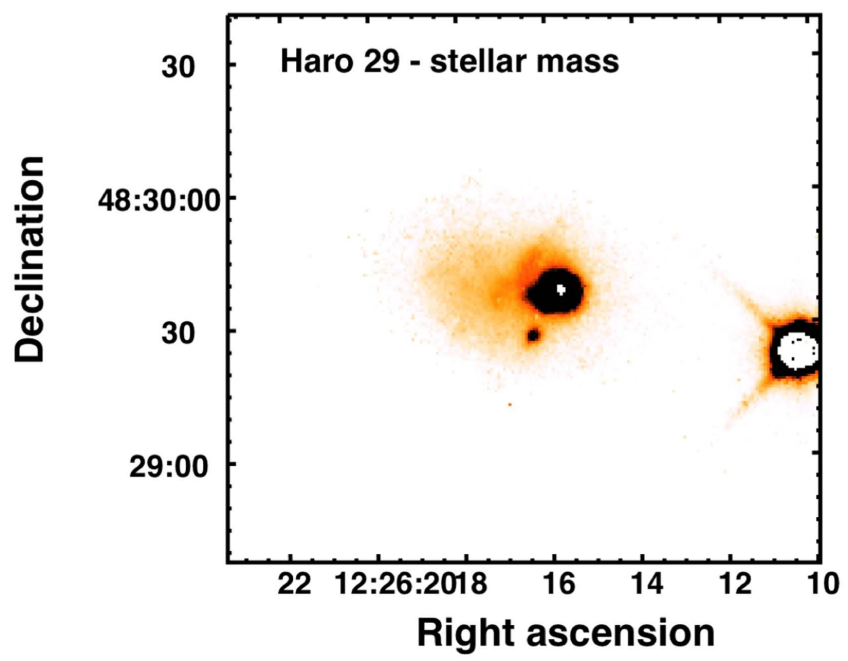

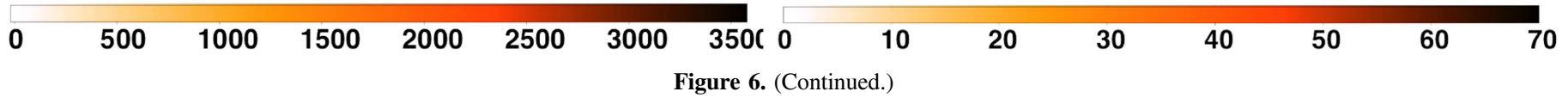

higher internal pressures, of order $4 \times 10^{-11} \mathrm{~g} \mathrm{~s}^{-2} \mathrm{~cm}^{-1}$ (Blitz \& Rosolowsky 2004), solidly in bin 3.

Therefore, we have maps of the pressure, gas mass surface density, and stellar mass surface density with which to characterize the environment in which a stellar object has formed. In order to associate a particular environment with a cluster, OB association, or O star, we divided the pressure maps into regions that sampled the different pressure environments of each galaxy. This was done by eye from the pressure maps, and each circle is meant to roughly select a region of similar pressure (i.e., brightness on the pressure map). The purpose of averaging over regions is to increase the signal-to-noise ratio and isolate high- and low-pressure areas. The pressure maps with the regions encircled are shown in Figure 7. All regions are shown even though many of them ended up not having clusters or $\mathrm{O}$ stars in them. F275W images of all of the galaxies are shown in Figures 8-12, with the clusters, OB associations, and $\mathrm{O}$ stars marked along with the pressure regions. We then measured the average pressure, gas mass surface density, and stellar mass surface density within each of these circles. The average values associated with a given circle are assigned to the clusters, $\mathrm{OB}$ associations, and $\mathrm{O}$ stars that fall within that circle. For those objects falling between circles, the closest circle is used.

From these figures, we note, first, that there are far more $\mathrm{O}$ stars than clusters in each galaxy. Second, clusters are not always found where the $\mathrm{O}$ stars are located. Third, $\mathrm{O}$ stars are often clustered, and these clusterings are what we have identified as OB associations.

\section{Results \\ 3.1. Cluster Characteristics}

\subsubsection{Characteristics as a Function of Galactic Properties}

In Figure 13 we plot the cluster CI and mass against their galactic environmental properties of pressure, stellar mass density, and H I surface mass density. Each galaxy is plotted with a different symbol, but note that DDO 63 has no clusters and is included in the legend as a reminder that it is a part of 


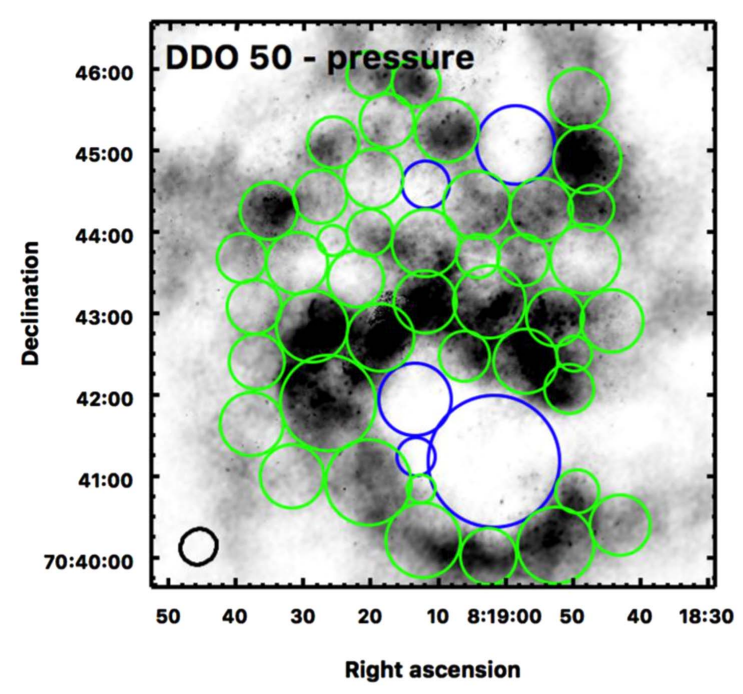

$2.70 e-12$
$2.40 e-12$
$2.10 e-12$
$1.80 e-12$
$1.50 e-12$
$1.20 e-12$
$9.00 e-13$
$5.98 e-13$
$2.99 e-13$
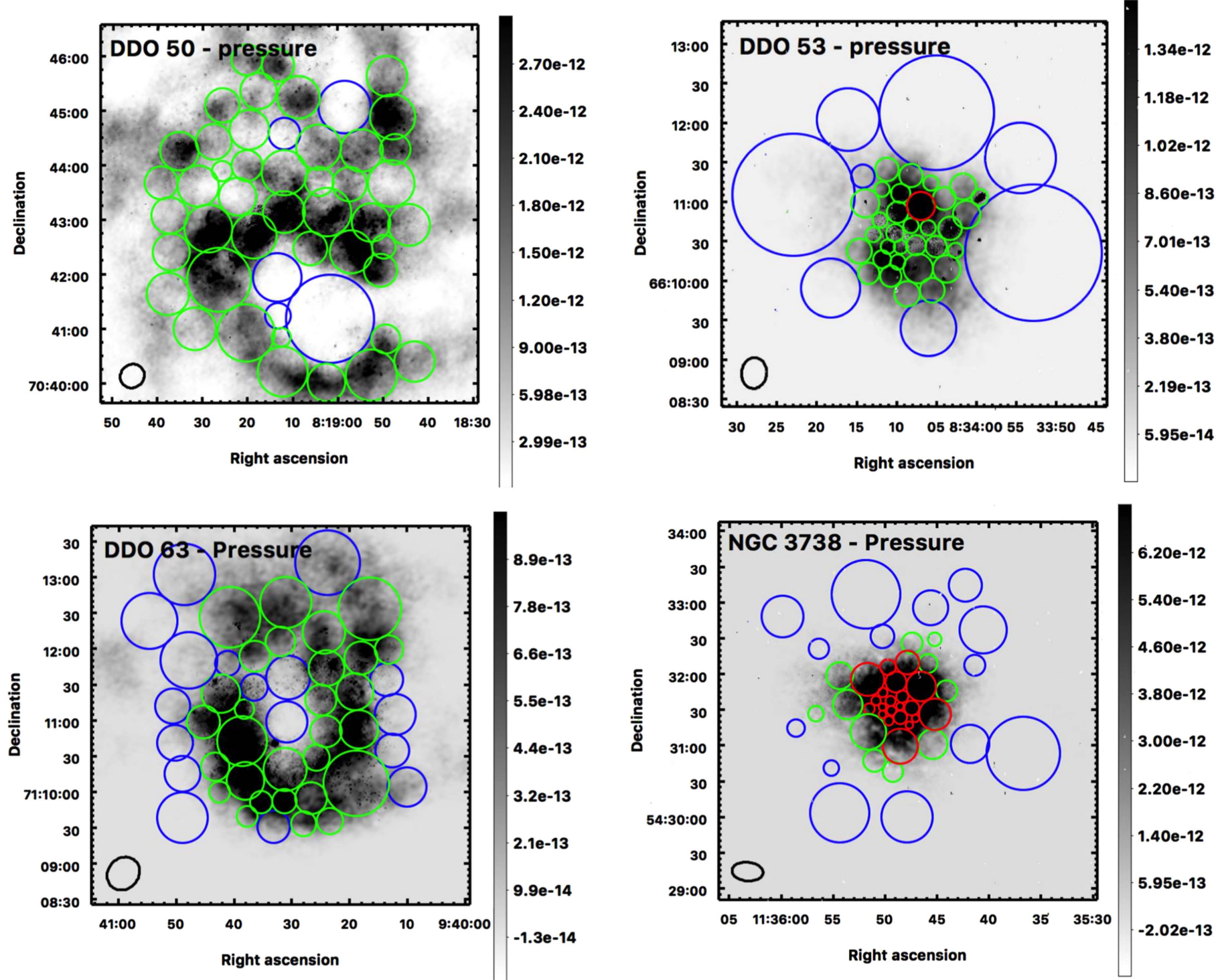

$8.9 e-13$
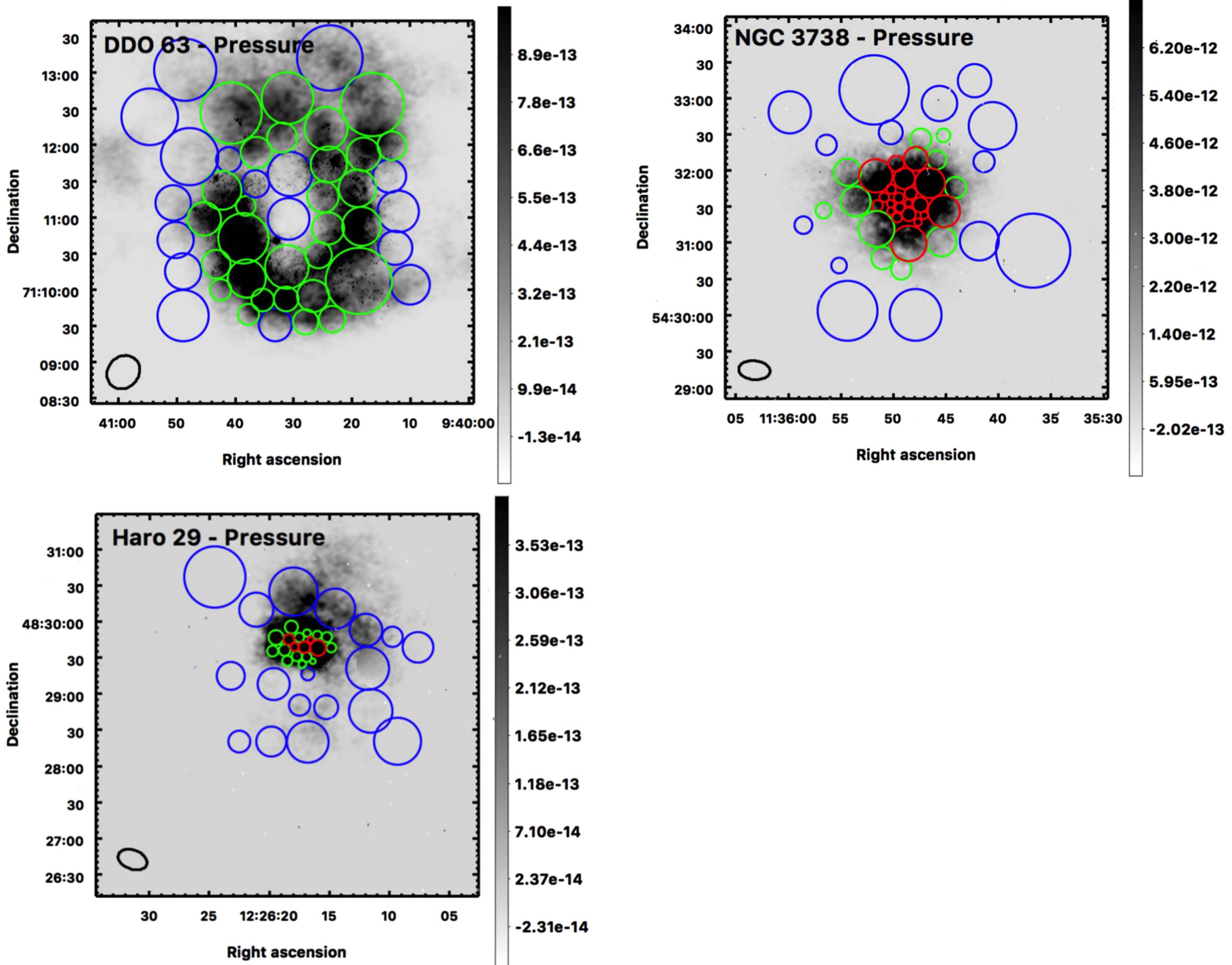

3.53e-13

$3.06 e-13$

2.59e-13

2.12e-13

$1.65 \mathrm{e}-13$

$1.18 \mathrm{e}-13$

7.10e-14

2.37e-14

$-2.31 e-14$

Figure 7. Pressure maps of the five galaxies showing the regions selected to sample the different pressure environment within the galaxy. The regions and their sizes were determined by eye from the pressure maps. The red circles are for regions with average pressures $\log P \geqslant-11.4$, green circles for average pressures $-12.4 \geqslant \log P<-11.4$, and blue circles for average pressures $\log P<-12.4$, where the units of $P$ are $\mathrm{g}\left(\mathrm{s}^{2} \mathrm{~cm}\right)^{-1}$. The images are displayed to show structure within the inner parts of the galaxy, although gas extends much further than is obvious in these images. The beam sizes of the H I maps that form the dominant component of the pressure maps are shown as black ellipses in the lower left corner of each panel. 


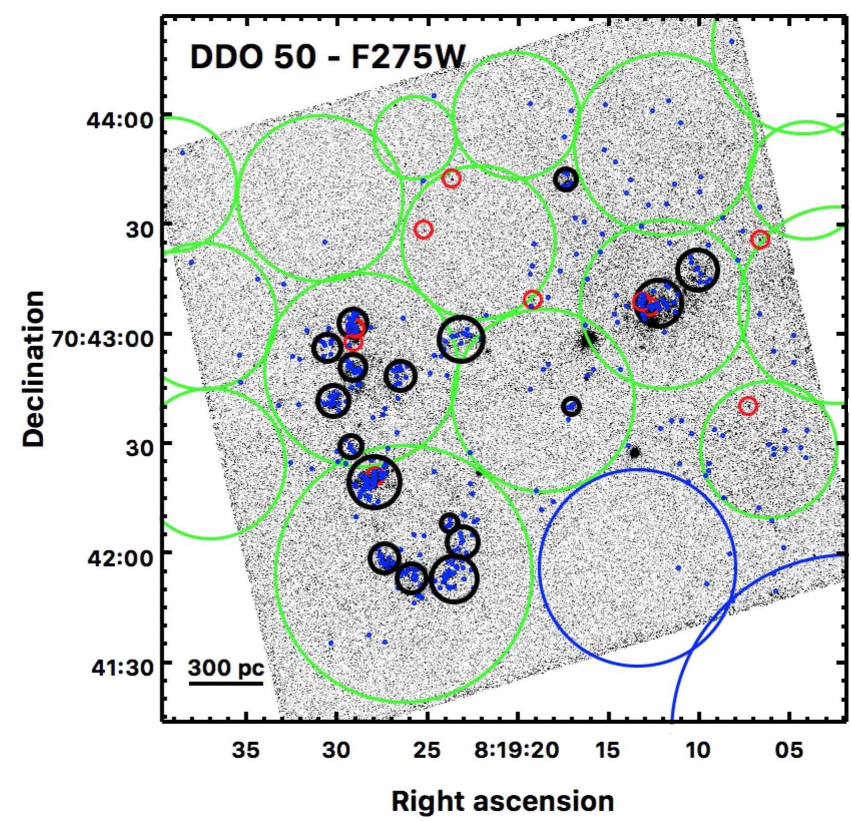

Figure 8. F275W image of DDO 50 showing the regions selected to sample the pressure environment within the galaxy, young compact clusters (small red circles), the $\mathrm{O}$ star candidates (small blue circles), and $\mathrm{OB}$ associations (black). The large green circles are for average pressures $-12.4 \geqslant \log P<-11.4$, and the blue circles are for average pressures $\log P<-12.4$, where the units of $P$ are $\mathrm{g}\left(\mathrm{s}^{2} \mathrm{~cm}\right)^{-1}$. There are no regions with $\log P \geqslant-11.4$. The pressure regions were determined by eye from the pressure maps; stars or clusters between pressure regions were assigned to the closest region. Clusters are nearly point-like, and the red circles do not represent the size of the cluster. The black circles indicate the size of the OB associations, determined by eye.

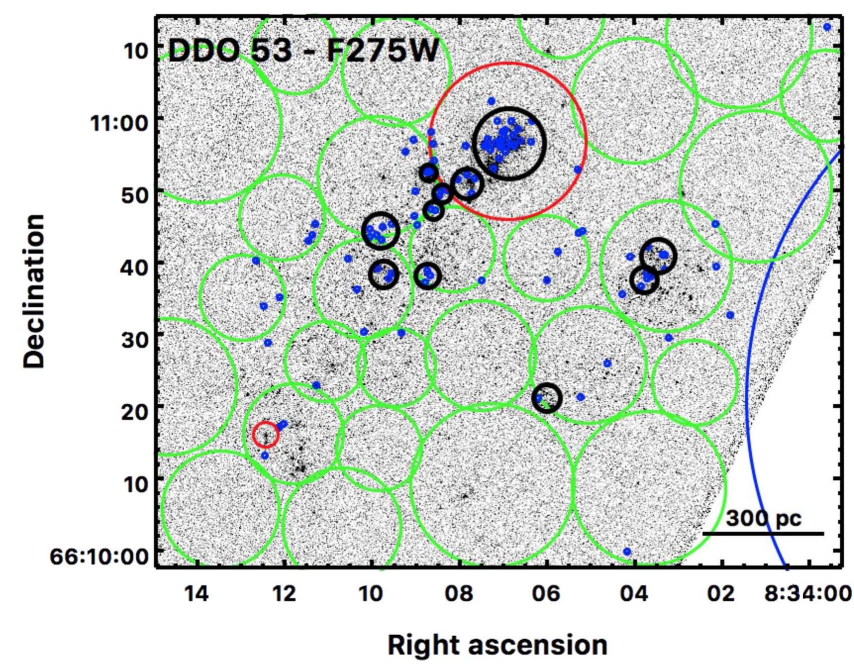

Figure 9. F275W image of DDO 53 showing the regions selected to sample the pressure environment within the galaxy, young compact clusters (small red circles), the $\mathrm{O}$ star candidates (small blue circles), and OB associations (black). The large red circles are for regions with average pressures $\log P \geqslant-11.4$, green circles for average pressures $-12.4 \geqslant \log P<-11.4$, and blue circles for average pressures $\log P<-12.4$, where the units of $P$ are $\mathrm{g}\left(\mathrm{s}^{2} \mathrm{~cm}\right)^{-1}$. Clusters are nearly point-like, and the red circles do not represent the size of the cluster. The black circles indicate the size of the OB associations, determined by eye.

this sample. We see that clusters are found at a wide range of pressures and $\mathrm{HI}$ surface densities. However, the clusters in NGC 3738 and Haro 29 are generally found at higher pressures, stellar mass densities, and $\mathrm{HI}$ surface densities than the clusters in the other two galaxies. Haro 29 is likely an

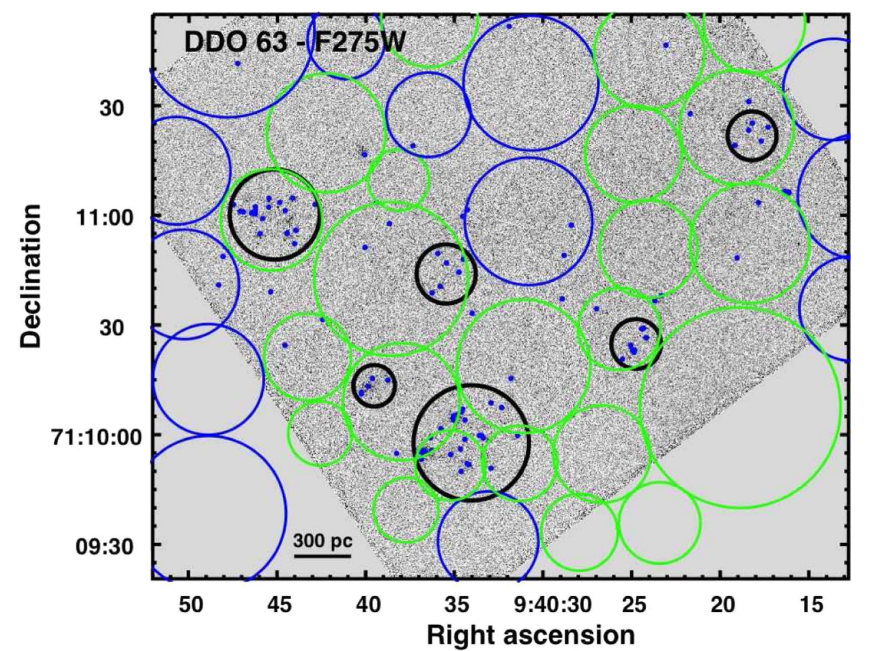

Figure 10. F275W image of DDO 63 showing the regions selected to sample the pressure environment within the galaxy, O star candidates (small blue circles), and $\mathrm{OB}$ associations (black). The large green circles are for average pressures $-12.4 \geqslant \log P<-11.4$, and blue circles are for average pressures $\log P<-12.4$, where the units of $P$ are $\mathrm{g}\left(\mathrm{s}^{2} \mathrm{~cm}\right)^{-1}$. There are no regions with $\log P \geqslant-11.4$ within this galaxy. The black circles indicate the size of the OB associations, determined by eye. DDO 63 has no clusters.

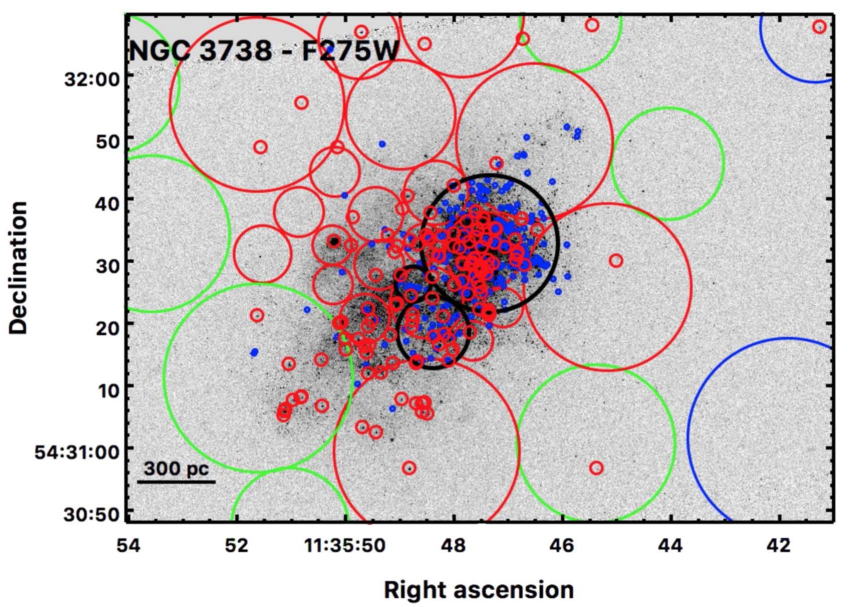

Figure 11. F275W image of NGC 3738 showing the regions selected to sample the pressure environment within the galaxy, young compact clusters (small red circles), the $\mathrm{O}$ star candidates (small blue circles), and $\mathrm{OB}$ associations (black). The large red circles are for regions with average pressures $\log P \geqslant-11.4$, green circles for average pressures $-12.4 \geqslant \log P<-11.4$, and blue circles for average pressures $\log P<-12.4$, where the units of $P$ are $\mathrm{g}\left(\mathrm{s}^{2} \mathrm{~cm}\right)^{-1}$. Clusters are nearly point-like, and the red circles do not represent the size of the cluster. The black circles indicate the size of the OB associations, determined by eye.

advanced dwarf-dwarf merger (Ashley et al. 2013), and NGC 3738 may be too (Ashley et al. 2017). Perhaps such external events are necessary to produce large numbers of clusters or extraordinary star-forming regions in dwarfs. The other three systems are more typical, likely internally driven dIrrs (Hunter et al. 2012; but see Bernard et al. 2012; Egorov et al. 2017, concerning DDO 50). We find only one compact cluster (NGC 3738's) in the lowest pressure range. In the pressure range where these dwarfs form compact and likely bound stellar systems, it does not show a trend of characteristics with increasing pressure.

To check whether a correlation between cluster characteristics and galactic environmental properties was being lost in 


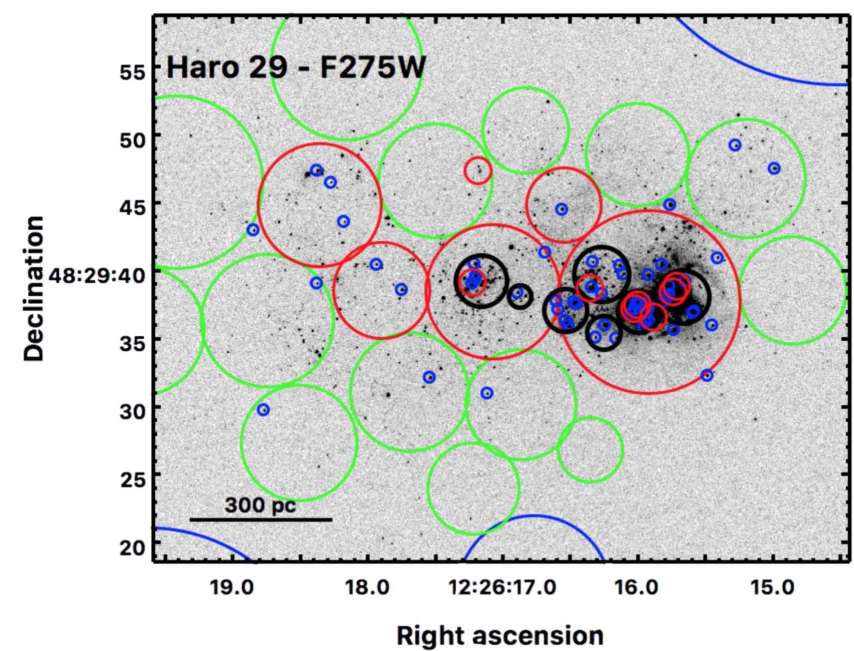

Figure 12. F275W images of Haro 29 showing the regions selected to sample the pressure environment within the galaxy, young compact clusters (small red circles), the $\mathrm{O}$ star candidates (small blue circles), and $\mathrm{OB}$ associations (black). The large red circles are for regions with average pressures $\log P \geqslant-11.4$, green circles for average pressures $-12.4 \geqslant \log P<-11.4$, and blue circles for average pressures $\log P<-12.4$, where the units of $P$ are $\mathrm{g}\left(\mathrm{s}^{2} \mathrm{~cm}\right)^{-1}$. Clusters are nearly point-like, and the red circles do not represent the size of the cluster. The black circles indicate the size of the $\mathrm{OB}$ associations, determined by eye.
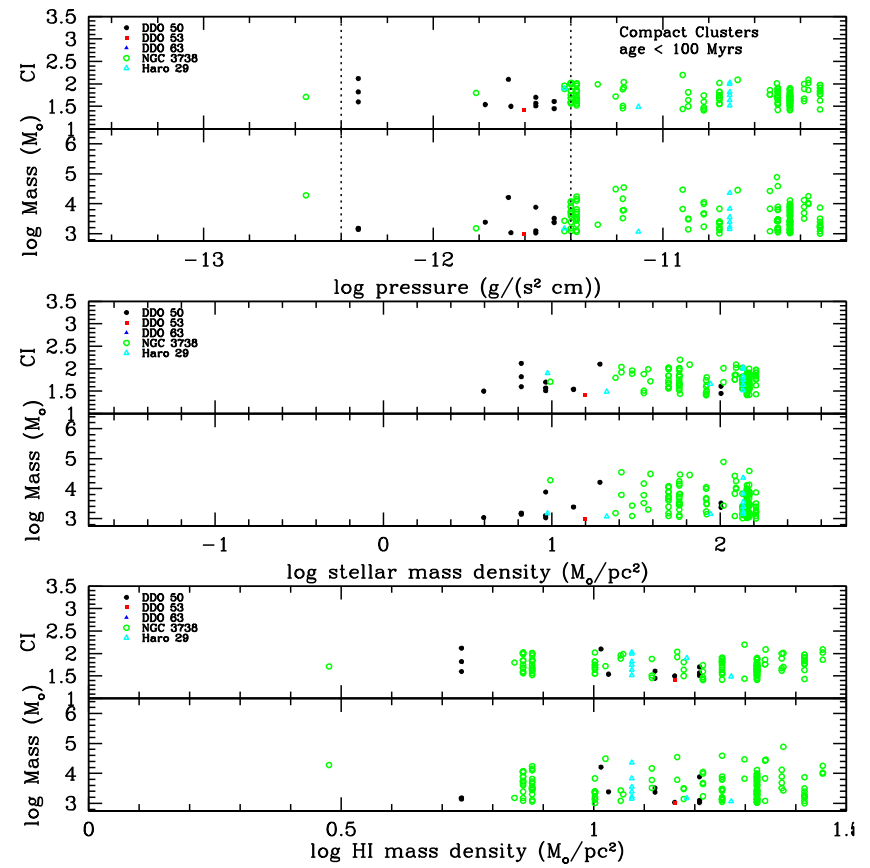

Figure 13. Compact cluster characteristics vs. galactic environment in which the clusters are found for clusters with ages less than $100 \mathrm{Myr}$. The cluster characteristics include cluster mass and CI. Galactic environmental characteristics include pressure, stellar mass density, and $\mathrm{H} \mathrm{I}$ mass surface density. The vertical dotted lines in the top panel delineate the three pressure bins discussed in the text (Section 3.1.2).

noisy cluster data, we produced Figure 13 for only cluster class 1 objects. These are clusters that are compact and bright and less ambiguous in their classification as a compact cluster than other objects. The numbers of clusters drops, but there is no trend with this subset of clusters.

Figure 13 is for all compact clusters with ages up to 100 Myr. In Figure 14 we plot the same quantities but only for
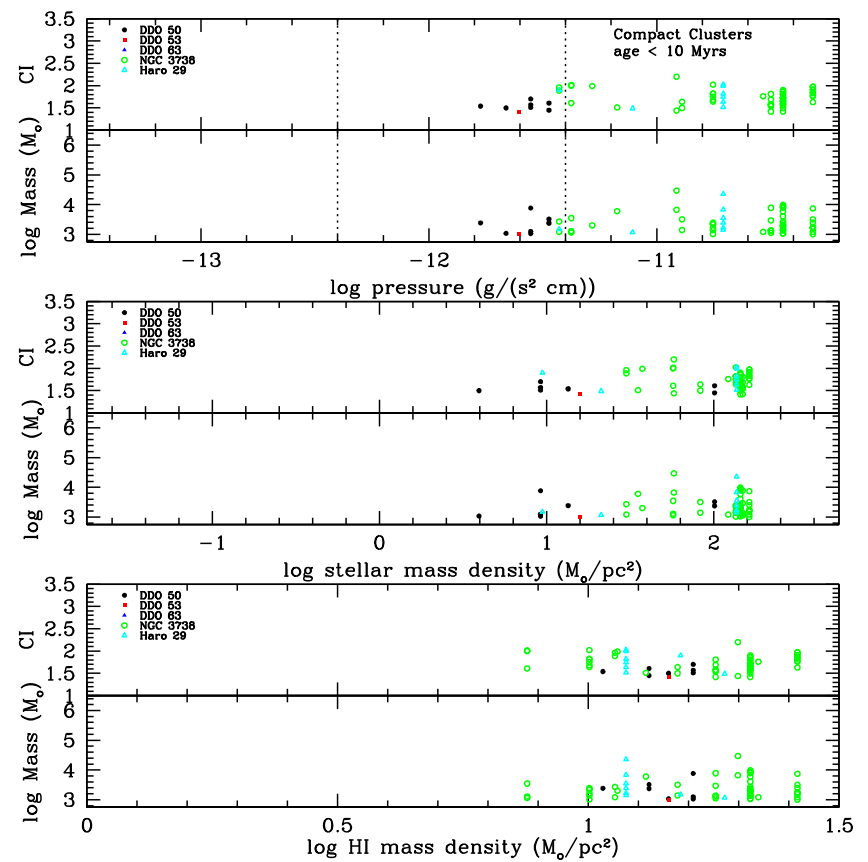

Figure 14. Compact cluster characteristics vs. galactic environment in which the clusters are found for clusters with ages less than $10 \mathrm{Myr}$. The cluster characteristics include cluster mass and CI. Galactic environmental characteristics include pressure, stellar mass density, and $\mathrm{H} \mathrm{I}$ mass surface density. The vertical dotted lines in the top panel delineate the three pressure bins discussed in the text (Section 3.1.2).

clusters with ages up to $10 \mathrm{Myr}$. There are fewer clusters (see Table 2), but again, no trend of cluster properties with environmental properties is seen.

Within the statistical uncertainties, the clusters cover the same range of properties independent of the galaxy or part of the galaxy in which they are found. This could imply that larger-scale effects are more important in determining the cluster characteristics, as proposed by the model of Whitmore et al. (2007; see also Whitemore 2017). Another possibility is that once the conditions for clustered star formation are reached, the gravitational collapse and the fragmentation properties of the interstellar medium drive the final star formation efficiency within the regions, thus making cluster formation a local and stochastic process (e.g., Longmore et al. 2014). Current studies of the ICMF show that it is described by a power-law function with slope close to -2 , consistent with the hierarchical fragmentation caused by the scale-free action of turbulence. Star formation in dIrr galaxies and BCDs is more sporadic than it is in spiral systems. Thus, the lack of a correlation may be caused by small number statistics in sampling the cluster mass function. The lack of dependence of cluster size on the midplane pressure could be explained if dynamical stellar processes, within the gravitationally bound regions where clusters formed, operate on very short timescales (e.g., Grudić et al. 2017), canceling any memory of the initial conditions. This fast dynamical evolution, which includes stellar feedback, merging of subclumps in the young cluster, and tidal stripping by the host galaxy, would explain why cluster sizes and surface brightness profiles do not depend on galactic environment, cluster age, and galaxy type (e.g., Grudić et al. 2017; Ryon et al. 2017).

One issue in comparing cluster characteristics with the cluster's environment is determining the true environment in which the cluster formed. We restrict ourselves to clusters with 

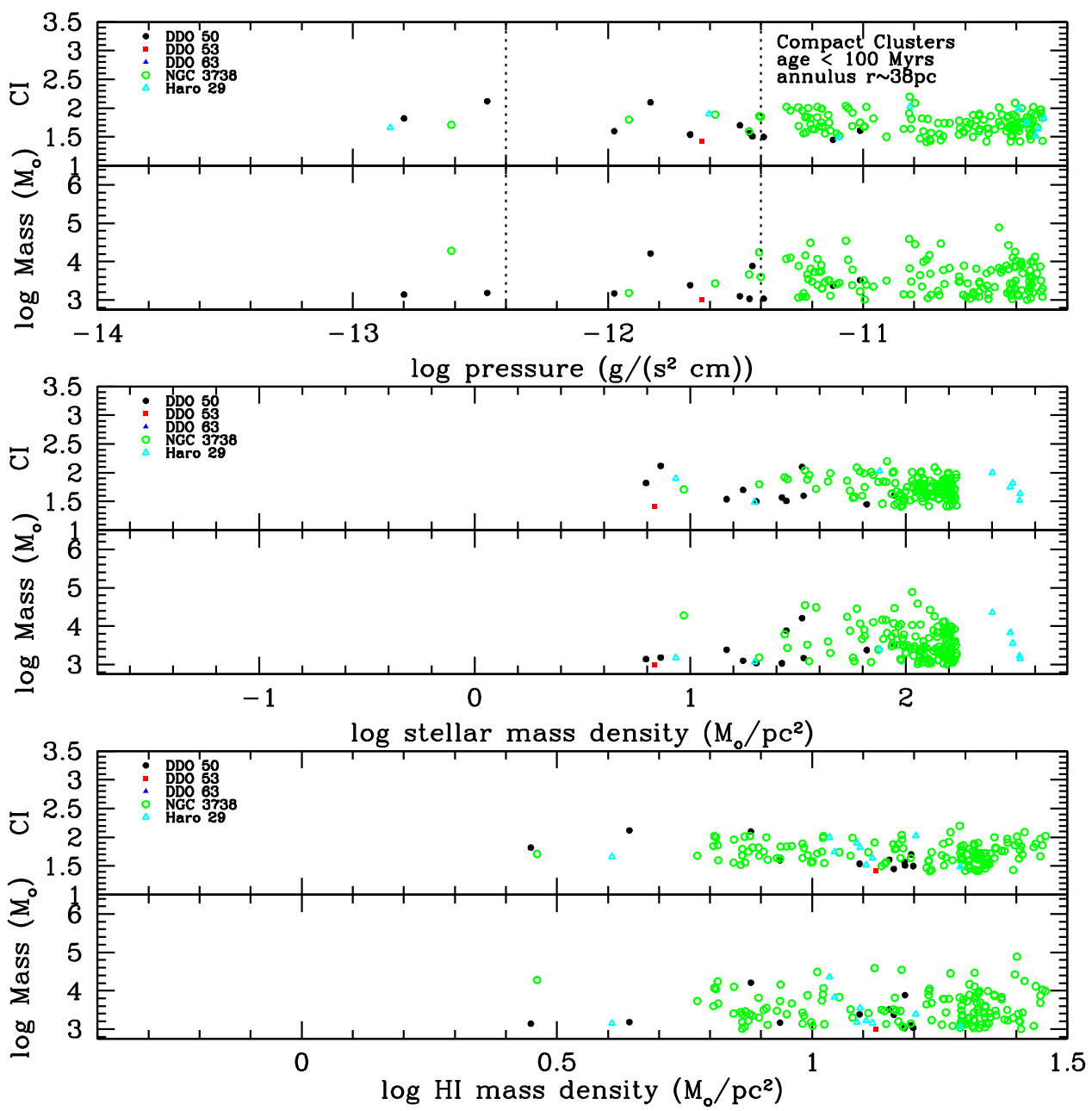

Figure 15. Comparison of cluster characteristics with galactic environment determined from the smallest annulus, $\sim 38 \mathrm{pc}$. This is similar to Figure 13 , but with the galactic environment determined from an annulus around the cluster rather than selected regions. The cluster characteristics include cluster mass and CI. Galactic environmental characteristics include pressure, stellar mass density, and H I mass surface density. The vertical dotted lines delineate the three pressure bins discussed in the text. The radius is the midpoint of the annulus. There is no trend with cluster characteristic. An animation of this figure is available. It starts with an annulus of $r=38 \mathrm{pc}$ and ends with an annulus of $r=950 \mathrm{pc}$. The duration is $2 \mathrm{~s}$.

(An animation of this figure is available.)

ages less than $100 \mathrm{Myr}$ in order to minimize to some extent the amount by which the environment has changed since cluster formation, although the formation of the cluster itself modifies the natal environment. In addition, we minimize the degree to which the clusters have dissolved (Lamers 2009; Baumgardt et al. 2013).

Above we chose to capture the environmental characteristics in regions defined by sampling of pressure maps. For comparison, we also determine the environment in annuli centered on each cluster with increasing distance from the cluster. We want to see whether there is any scale at which a correlation becomes apparent. We define a region around each cluster to be eliminated from the environmental measurements as a circle with a radius of $25 \mathrm{pc}$ so that we are not including the cluster or $\mathrm{OB}$ association itself. We then measure the average pressure, gas mass surface density, and stellar mass surface density in circles of radii $25-150 \mathrm{pc}$ in steps of $25 \mathrm{pc}$, $150-300 \mathrm{pc}$ in steps of $50 \mathrm{pc}$, and $300 \mathrm{pc}-1 \mathrm{kpc}$ in steps of $100 \mathrm{pc}$, for a total of 15 circles. Annuli were constructed as the area between two successive circles.

We made plots like Figure 13 for each environmental annulus and constructed an animated ensemble of the plots in order to easily see the changes with radius. The movie is available in the online materials associated with this paper. In Figure 15 we show the same panels as in Figure 13, but for the smallest annulus, $\sim 38 \mathrm{pc}$ radius. This explores the environment immediately around the clusters, which can contain other starforming units. In Figure 16 we also plot the cluster characteristics against pressure for annuli 1, 7, and 15 (radii of 38,225 , and $950 \mathrm{pc}$, respectively) as an illustration of the entire range of radii. We find that the environmental characteristics change with annulus, steadily becoming lower in value with increasing radius. Not only is each annulus further from the cluster with increasing radius, but the area over which the galactic characteristics are measured increases with radius. From the first annulus to the last there is a factor of 100 increase in area. Thus, the lower values of the larger radii annuli are likely due to averaging over a larger area, essentially smoothing out peaks and valleys. Nevertheless, there is no radius at which a trend of cluster characteristics with environment develops.

In Figure 17 we also compare for each cluster the environmental characteristics measured in the regions shown in Figure 7 with the environmental characteristics measured in the 

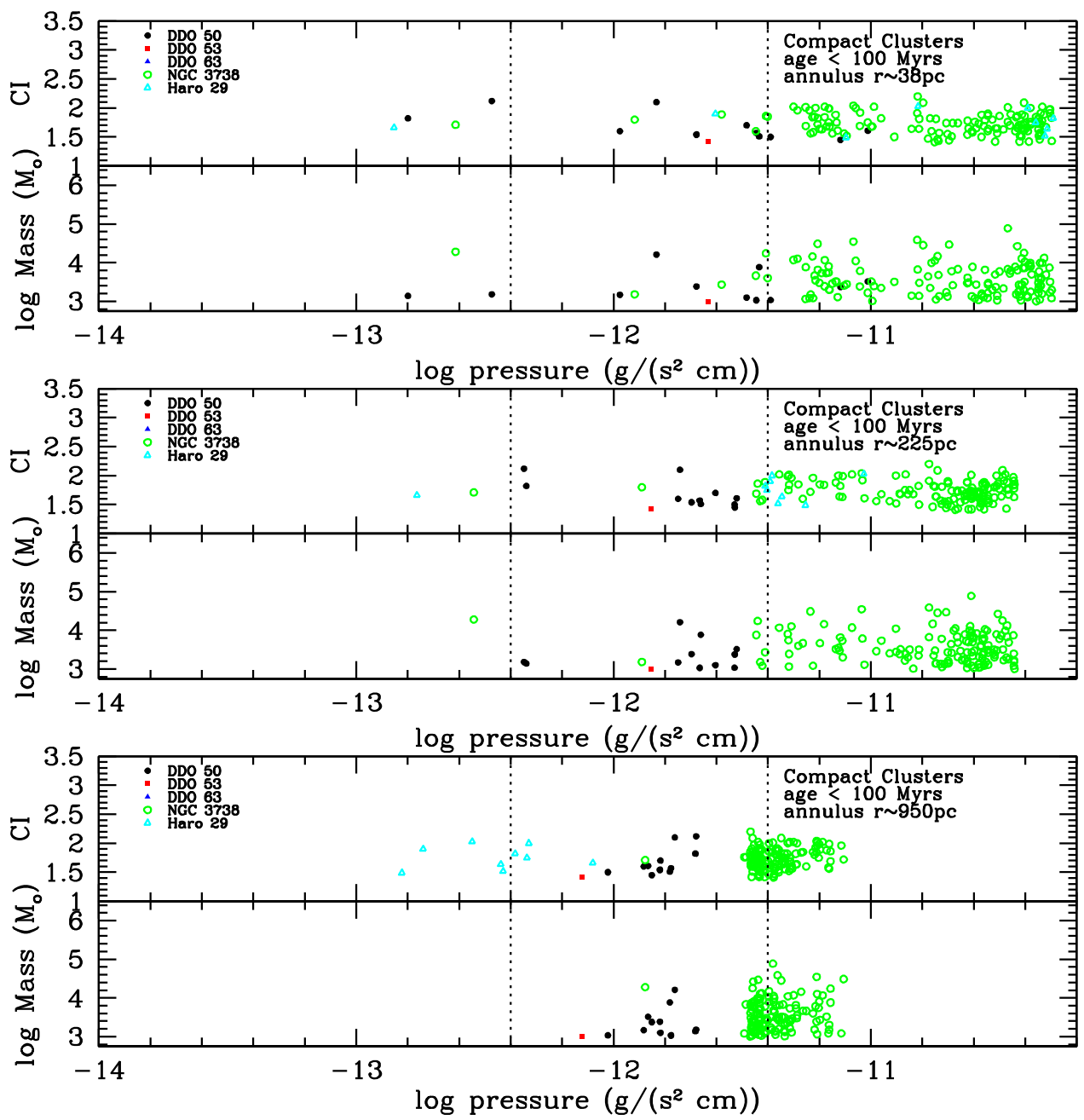

Figure 16. Extract of a movie comparing cluster characteristics with galactic environment determined from annuli of progressively larger distance from the cluster. This is similar to Figure 13, but with the galactic environment determined from annuli rather than selected regions. The cluster characteristics include cluster mass and CI. Galactic environmental characteristics include pressure, stellar mass density, and H I mass surface density, although here we only show the pressure panels. The vertical dotted lines delineate the three pressure bins discussed in the text. The movie shows 15 annuli from 38 to 950 pc. Here we show the first, seventh, and last annuli. One can see that the pressure changes with the area of the galaxy being sampled, so that clusters at one pressure in the upper panel will appear at a different pressure in the lower panel. The radius is the midpoint of the annulus. There is no trend with cluster characteristic at any radius.

annulus immediately around the cluster. There is a one-to-one relationship with scatter. Since we are after the environmental parameters in which the cloud formed that made the clusters, we prefer the regional characteristics that provide a reasonable average over conditions rather than characteristics determined in the close-in annulus that is subject to local variations and crowding of other recent star formation.

\subsubsection{Characteristics by Pressure Region}

To look at the role of pressure in another way, we examine the clusters of the pressure regions of Figure 7 in three bins; bin 1 is $\log$ pressure $<-12.4$, bin 2 is $\log$ pressure between -12.4 and -11.4 , and bin 3 is $\log$ pressure $>-11.4$, where units of pressure are $\mathrm{g}\left(\mathrm{s}^{2} \mathrm{~cm}\right)^{-1}$. These bins were chosen by eye based on groupings of clusters and are marked with vertical dashed lines in Figures 13-16. Note that we are only including the regions outlined in Figure 7 . These regions were chosen primarily to cover the parts of the galaxies where the stars and clusters are located, mostly the central regions, and they do not include all of the gas associated with each galaxy, particularly extended low-density gas. Furthermore, the pressure by which the region is binned is the average within each region. The purpose here is to describe and compare the identified pressure regions.

In the top panels of Figure 18 we plot the fraction of the total area covered by each pressure bin (panel (a)) and the fraction of the H I gas contained in each pressure bin (panel (d)). We see, for example, that although the clusters in NGC 3738 and Haro 29 are found mostly in pressure bin 3 , the fraction of area occupied by these pressure regions is not high, 15\%-18\%. On the other hand, in NGC 3738 bin 3 contains the majority of the gas, in contrast to what is seen in the rest of the systems. For the three typical dIrrs (DDO 50, DDO 53, DDO 63) the majority of the gas is in pressure bin 2, and none to very little is in bin 3. Haro 29 is opposite to both of these trends, with the majority of its gas in bin 1, the lowest pressure. In terms of cluster characteristics, the number of clusters (panels (b) and (e)) and total mass in clusters (panels (c) and (f)) per unit area and per unit gas mass generally increase from pressure bin 2 to pressure bin 3 for the two galaxies with clusters in both bins 2 and 3-NGC 3738 and Haro 29. (DDO 63 has no clusters, and DDO 50 and DDO 53 only have clusters in bin 2.) We examine 

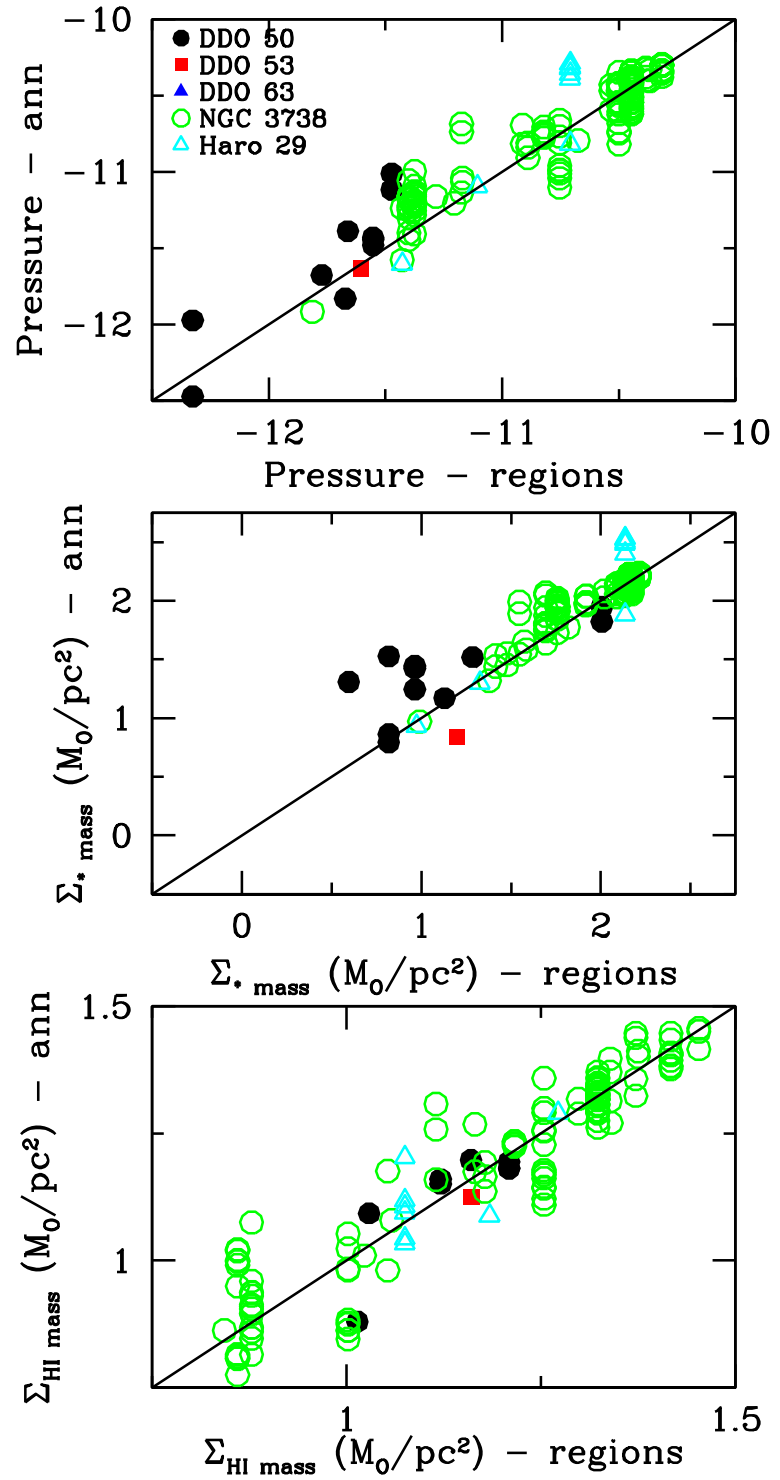

Figure 17. Comparison of galactic environmental characteristics determined from the smallest annulus, $\sim 38 \mathrm{pc}$, with values determined from averages over the regions shown in Figure 7 for each compact cluster. Galactic environmental characteristics include pressure, stellar mass density, and $\mathrm{HI}$ mass surface density. The solid line is a one-to-one equality of the characteristics, and the relationship is one-to-one with scatter. Since we are after the environmental parameters in which the cloud formed that formed the clusters, we prefer the regional characteristics that provide a reasonable average over conditions rather than characteristics determined in the close-in annulus that is subject to local variations and crowding of other recent star formation.

pressure bin 3 further by plotting, just for that pressure bin, the ratio of the cluster mass to $\mathrm{H}$ I mass against the fraction of the H I mass in pressure bin 3 in Figure 19. We see that the ratio of cluster mass to $\mathrm{H} \mathrm{I}$ mass is independent of the fraction of the $\mathrm{H} \mathrm{I}$ mass in pressure bin 3 , although only two galaxies have clusters in bin 3 .

We were curious what the pressure distribution was among the larger sample of LITTLE THINGS dIrrs, and to look at that, we binned the pressure and integrated H I maps on a pixel-bypixel basis for 29 of the LITTLE THINGS galaxies. Thus, here we include all of the gas, including low-density gas in the outer parts. Looking at the pressures on a pixel-by-pixel basis, we can investigate the full range of pressure environments in our galaxies and without averaging out the highs and lows as is a consequence of our analysis of selected regions. We found that $30 \%$ (12) of the galaxies have no gas in pressure bin 3, while another $30 \%$ have $>3 \%$ of their gas in this bin. Of the 40 LITTLE THINGS galaxies, NGC 3738 has the highest percentage of all of its gas in bin 3,26\%. The starburst galaxies NGC 1569 and IC 10 have $14 \%$ and $10 \%$ of their gas in bin 3, respectively. The other galaxies in this study-DDO 50, DDO 53, DDO 63, and Haro 29-have 0.8\%, 1.9\%, 0\%, and $2 \%$, respectively. We examined the LITTLE THINGS sample for any correlation between the integrated SFR and percentage of gas in pressure bin 3 and found none.

In Figure 20 we compare the identified clusters to the SFR measured from the SFR maps by pressure bin. First, panel (a) compares the total SFR per unit area in the three pressure bins, while panel (b) compares the unnormalized SFR in the different bins. Even though DDO 63 has no clusters, it does have FUV emission, and so it has a measured SFR in bins 1 and 2. We see that generally the higher-pressure bins have a higher SFR surface brightness. In panel (c) we compare the total mass in clusters divided by the SFR in the three pressure bins. There are two galaxies with clusters in all three pressure bins: NGC 3738 and Haro 29. In Haro 29 the mass formed in clusters divided by the SFR is approximately a constant with pressure. For NGC 3738 the ratio is lower at intermediate pressures (bin 2) than at higher pressures (bin 3), and at low pressure (bin 1) there is only one cluster, so statistics are poor there. The other two galaxies with clusters only in bin 2 have ratios that are comparable to NGC 3738's value in that bin. However, this suggests that the increase in the mass formed in clusters as a function of pressure shown in Figure 18(c), is mainly a reflection of the fact that both the mass formed in clusters and the SFR are higher in higher-pressure regions (see also Blitz \& Rosolowsky 2006). Figure 20(c) is also consistent with the finding by Chandar et al. (2015) that global cluster mass functions correlate with SFRs. In other words, the sampling of the cluster mass function is a stochastic process driven by sizeof-sample effects, and higher SFR enables sampling the cluster mass function at the high-mass end (e.g., Adamo \& Bastian 2015).

\subsubsection{Cluster Formation Rate}

We have estimated $\Gamma$, the ratio of cluster formation rate to integrated SFR, for clusters in each of the three pressure bins. This is not the individual circular pressure regions of Figure 7, but the sum of the clusters whose environmental pressures fall into the three pressure bins defined in Section 3.1.2. We have included only clusters from the team catalogs that have classes of 1 or 2 since these are compact clusters and more likely than multiple-peaked class 3 objects to be bound. We also include clusters with masses greater than or equal to $1000 M_{\odot}$ and ages less than or equal to $100 \mathrm{Myr}$. We extrapolate the mass in clusters with mass between 100 and $1000 M_{\odot}$ assuming that the cluster mass function is described by a power-law function with slope -2 . We do not exclude very young clusters, as the tracer used to derive the SFR is sensitive to star formation between 1 and 100 Myr. In bins with at least two clusters, we estimate the cluster formation rate as the total observed stellar mass divided by the age interval of $100 \mathrm{Myr}$. The uncertainty in $\Gamma$ takes into account the Poisson statistics of the numbers of clusters and the uncertainties associated with the cluster mass and age (e.g., see Adamo et al. 2015, for a complete description of the method). For pressure bins that contain less than two clusters, $\Gamma$ is not 

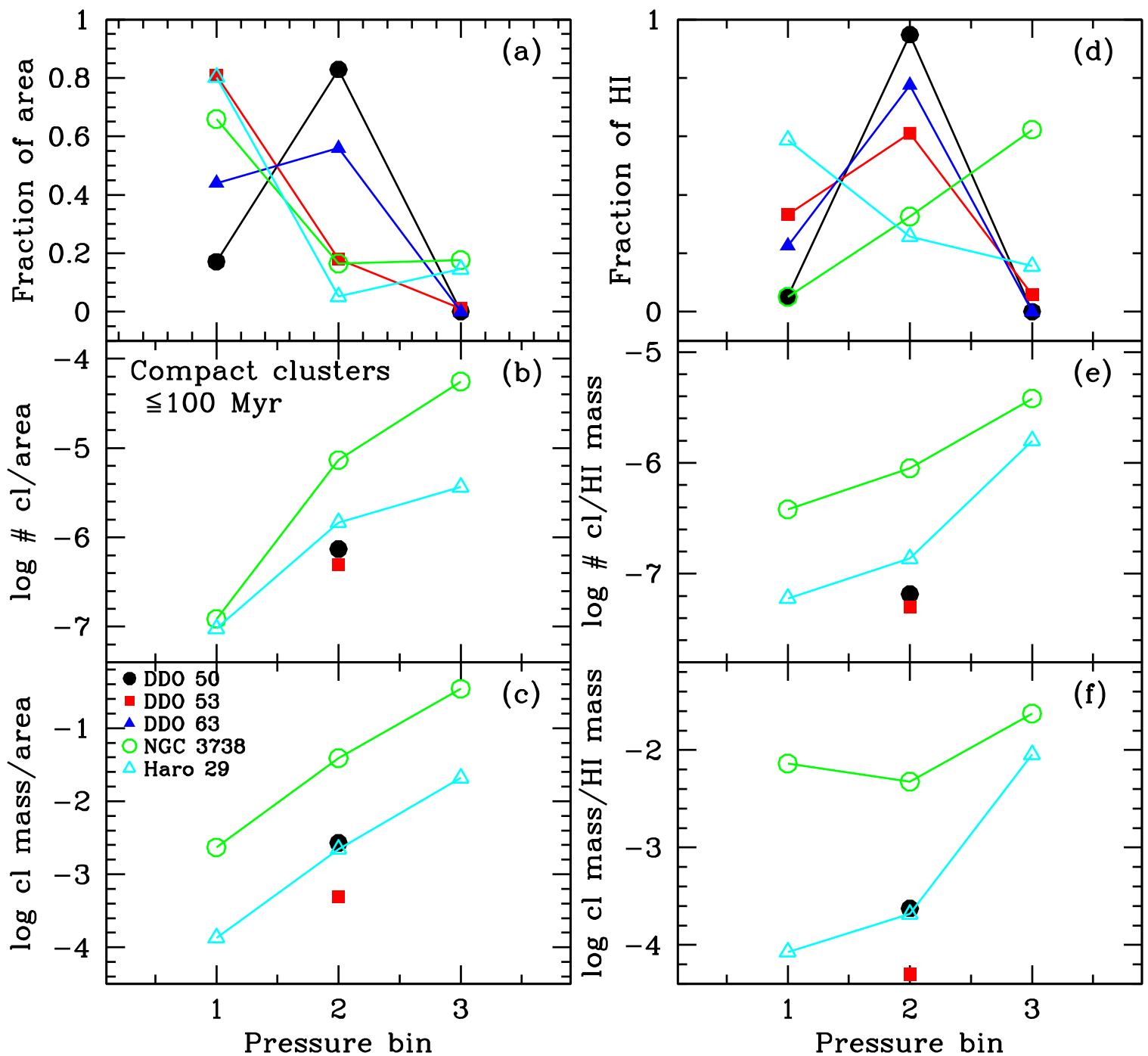

Figure 18. Number (panels (b) and (e)) and total mass (panels (c) and (f)) of clusters per unit area (panels (a)-(c)) and per H I gas mass (panels (d)-(f)) vs. pressure in which the clusters are found. The pressures fall in three bins: bin 1 is $\log$ pressure $<-12.4$, bin 2 is log pressure between -12.4 and -11.4 , and bin 3 is $\log$ pressure $>$ -11.4. Units of pressure are $\mathrm{g}\left(\mathrm{s}^{2} \mathrm{~cm}\right)^{-1}$, units of area are $\mathrm{pc}^{2}$, and units of mass are $M_{\odot}$. The area (panel (a)) is the total area of pressure regions shown in Figure 7 within the given bin range, and similarly for the H I mass (panel (d)). DDO 63 does not contain any clusters, and DDO 50 and DDO 53 do not have any clusters in pressure bins 1 and 3.

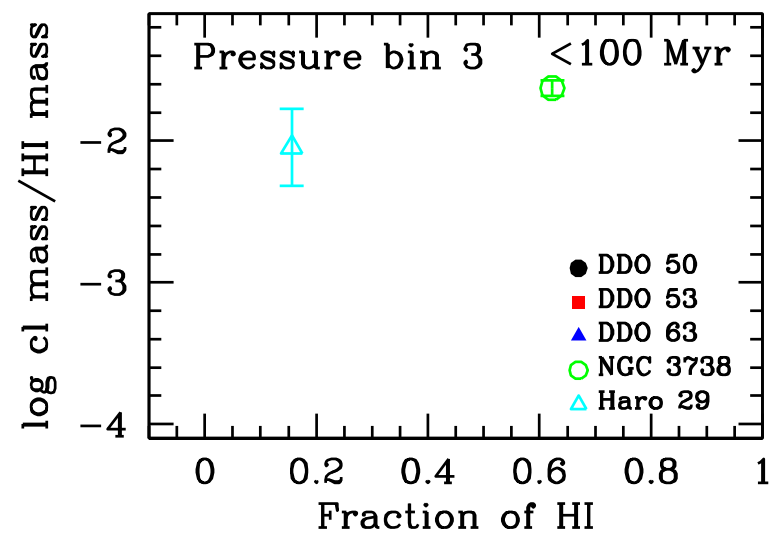

Figure 19. For pressure bin 3 only, total mass of clusters per H I gas mass vs. fraction of $\mathrm{HI}$ mass in this pressure bin. Pressure bin 3 is $\log$ pressure $>-11.4$. Units of pressure are $\mathrm{g}\left(\mathrm{s}^{2} \mathrm{~cm}\right)^{-1}$. DDO 63 does not contain any clusters, and DDO 50 and DDO 53 do not have any clusters in pressure bin 3 . The ratio of cluster mass to $\mathrm{HI}$ mass is constant for a range in fraction of H I mass. calculated. Issues related to the inconsistency in the timescale over which $\Gamma$ and $\Sigma_{\mathrm{SFR}}$ are determined are discussed by D. O. Cook et al. (2018b, in preparation), particularly in relation to dwarf galaxies.

In Figure 21 we plot $\Gamma$ as a function of pressure bin (left panel) and as a function of the SFR density (right panel) in the pressure bin where $\Gamma$ was calculated. $\Gamma$ varies from $0.9 \%$ to $33 \%$ in bin 2 and from $4 \%$ to $24 \%$ in bin 3 . NGC 3738 in pressure bin 2 has a $\Gamma$ that is significantly higher for that pressure than for DDO 50. For NGC 3738, with clusters in both pressure bins 2 and $3, \Gamma$ does not change significantly between the two pressure bins. Furthermore, $\Gamma$ does not show a correlation with the total SFR density measured by pressure bin, as shown in Figure 20(c). However, our dIrrs do scatter around the sequence of $\Gamma$ versus SFR density found in other galaxies (e.g., Goddard et al. 2010; Adamo et al. 2011, 2015; Annibali et al. 2011; Ryon et al. 2014; Lim \& Lee 2015; Johnson et al. 2016): for the intermediate-pressure bin (green squares) one galaxy lies above the black curve and one lies near 

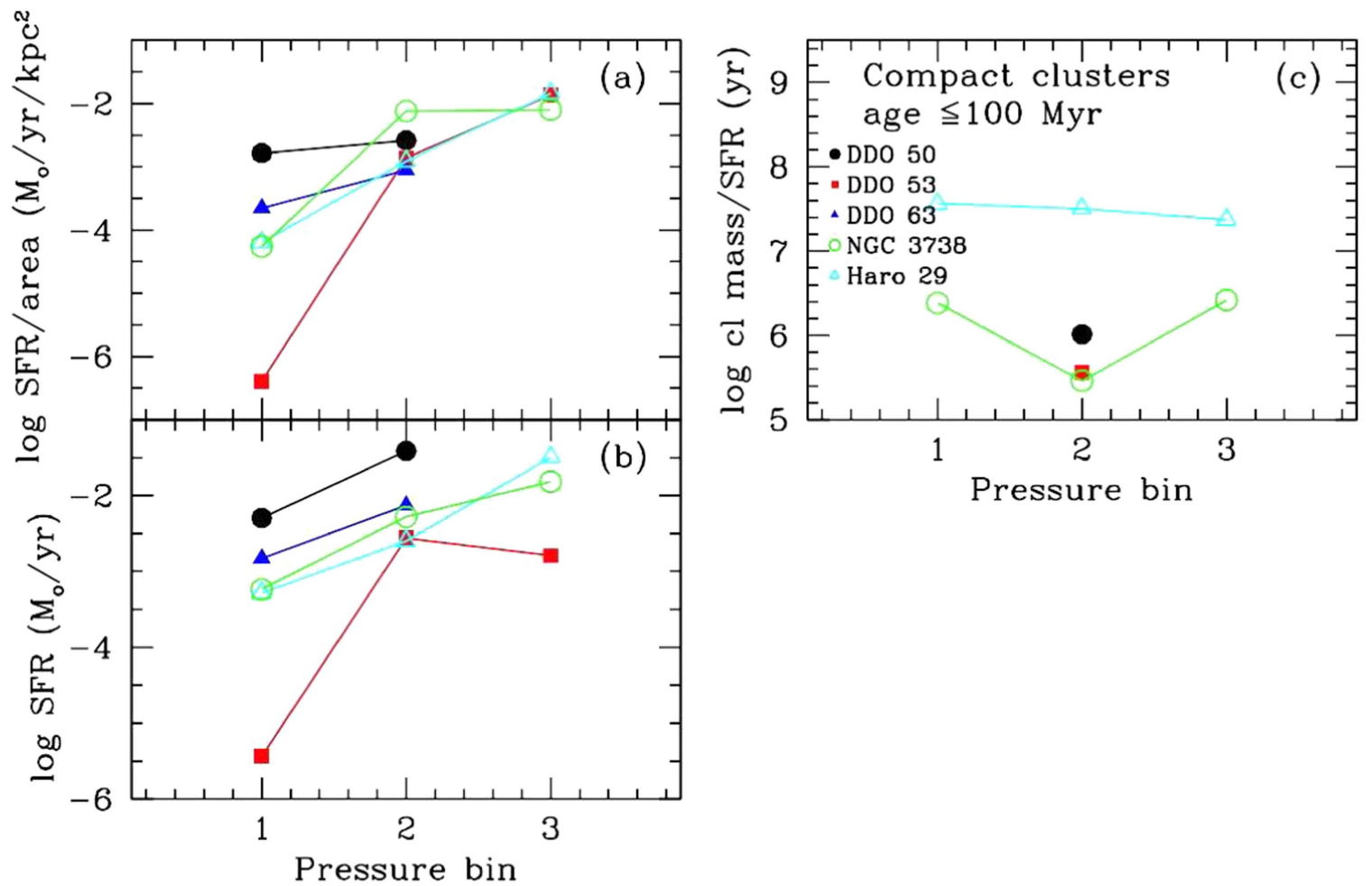

Figure 20. (a) SFR per unit area by pressure bin. (b) SFR by pressure bin. (c) Total cluster mass divided by the SFR by pressure bin. The pressures within each galaxy fall in three bins: bin 1 is $\log$ pressure $<-12.4$, bin 2 is $\log$ pressure between -12.4 and -11.4 , and bin 3 is $\log$ pressure $>-11.4$. Units of pressure are $\mathrm{g}\left(\mathrm{s}^{2} \mathrm{~cm}\right)^{-1}$, units of SFR are $M_{\odot} \mathrm{yr}^{-1}$, and units of mass are $M_{\odot}$. The area is the total area of pressure regions shown in Figure 7 within the given bin range. DDO 63 does not contain any clusters, and DDO 50 does not have any clusters in pressure bins 1 and 3. None of the galaxies have clusters in pressure bin 1 .
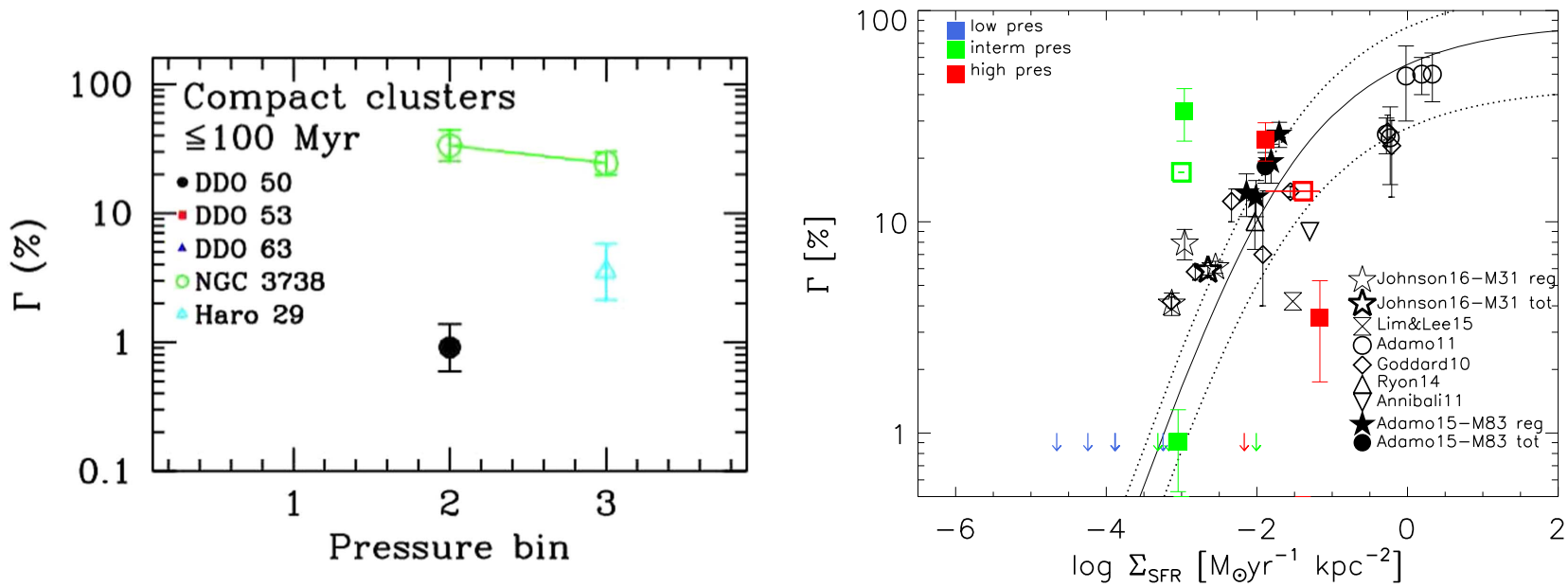

Figure 21. Left: $\Gamma$, the ratio of cluster formation rate to SFR, plotted on a logarithmic scale by pressure bin. Pressure bin 1 is $\log$ pressure $<-12.4$, bin 2 is $\log$ pressure between -12.4 and -11.4 , and bin 3 is log pressure $>-11.4$. Units of pressure are $\mathrm{g}\left(\mathrm{s}^{2} \mathrm{~cm}\right)^{-1}$. The area included is the total area of pressure regions shown in Figure 7 within the given bin range. Right: $\Gamma$ plotted on a logarithmic scale as a function of the SFR per unit area in the pressure bin where $\Gamma$ was calculated. The three pressure bins in each galaxy are plotted as filled squares color-coded by pressure bin in the upper left corner. The open squares are the averages of the galaxies in that pressure bin. Pressure bins that have fewer than two clusters are shown as upper limits along the $x$-axis. Other samples are shown in black according to the legend on the bottom right of the plot. The solid black line is the model of Kruijssen (2012) for the formation of bound clusters, and the dotted lines are the $1 \sigma$ uncertainties.

the curve. In the highest-pressure bin (number 3 , red squares in Figure 21, right panel) NGC 3738 lies near the black curve and Haro 29 lies well below. In the same plot, we also include the measured SFR densities (arrows) of the regions of galaxies that do not have any estimate of $\Gamma$, color-coded according to pressure bin. The range of SFR densities for low-pressure bins (blue arrows) reaches 1 mag lower than the SFR density values where clusters are formed. The SFR density range of intermediate- and high-pressure bins that form or do not form clusters (green and red arrows and squares) spans about two orders of magnitude, suggesting that cluster formation may still be highly stochastic, probably because of the episodic nature of star formation in dwarf galaxies. There are other suggestions that $\Gamma$ measured globally for galaxies is constant (see, e.g., Chandar et al. 2015, 2017). In that context, DDO 50 and Haro 29 are significantly different from NGC 3738 in this 


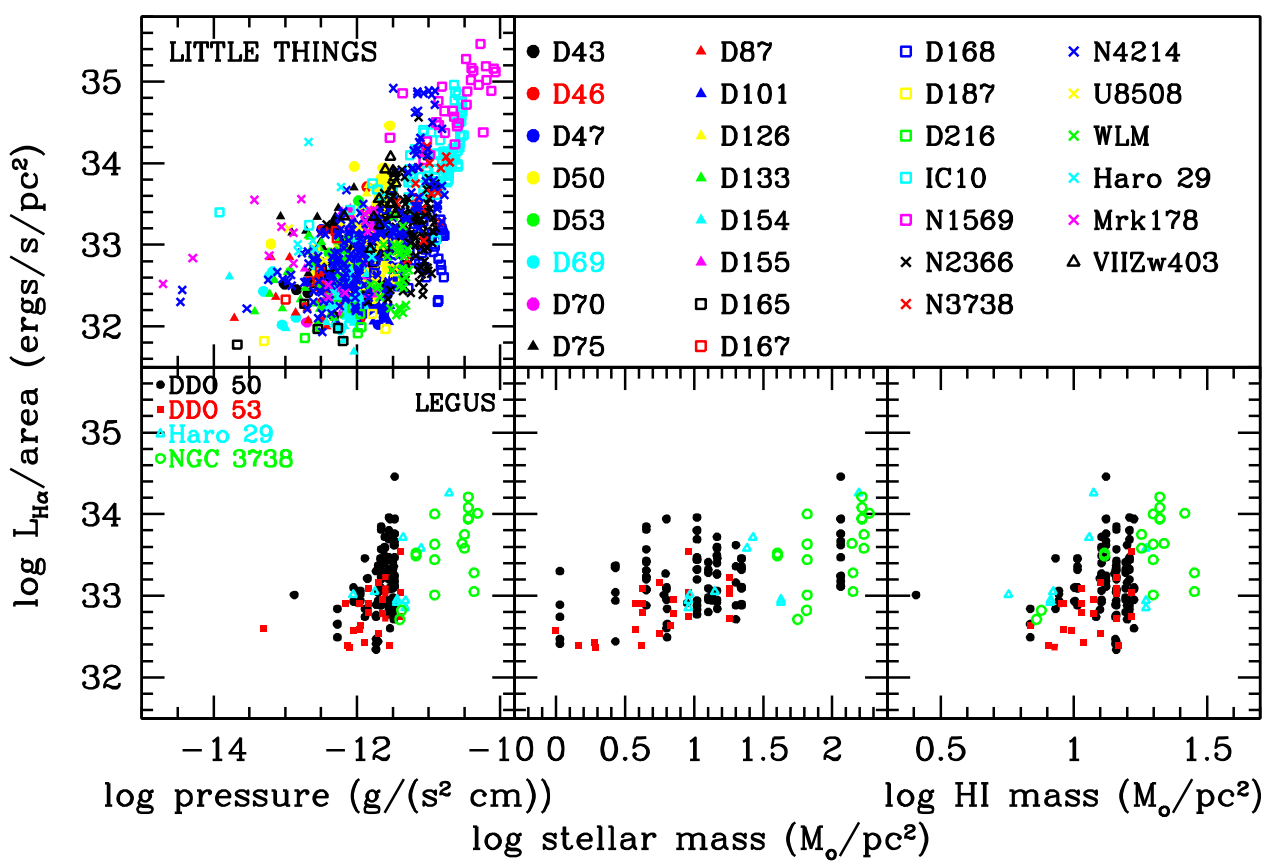

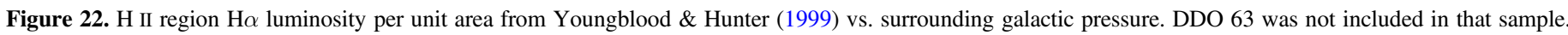

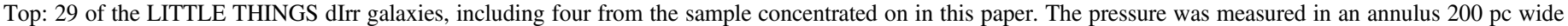

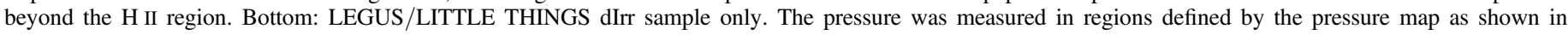
Figure 7.

sample. In D. O. Cook et al. (2018b, in preparation) we will present global values of $\Gamma$ calculated for these dwarfs and discuss the effects of using averaged SFR densities derived with calibrated flux conversions and SFR densities derived using stellar counts and resolved star formation histories. While the $\Gamma$ used in this work are estimated using an age range sensitive to the SFR tracer adopted, resolved recent star formation histories will enable us to estimate $\Gamma$ within smaller age ranges (D. O. Cook et al. 2018b, in preparation).

\subsection{H II Regions}

For many of the LITTLE THINGS galaxies, including four of the galaxies in this paper, we also have catalogs of $\mathrm{H}$ II regions to a completeness limit of about $2 \times 10^{32} \mathrm{erg} \mathrm{s}^{-1} \mathrm{pc}^{-2}$ (Youngblood \& Hunter 1999). These represent very young star-forming units, where the surrounding galaxy has not had much time to change since the formation of the stars in the H II region. Therefore, we made the equivalent of Figure 13 for the $\mathrm{H}$ II regions, which we present in Figure 22. We characterize the $\mathrm{H}$ II regions by the $\mathrm{H} \alpha$ surface brightness: the integrated $\mathrm{H} \alpha$ luminosity divided by the area covered by the H II region (see Youngblood \& Hunter 1999, for details). The diameters of the $\mathrm{H}$ II regions range from 10 to 500 pc. In Figure 22 the bottom panels show the $\mathrm{H} \alpha$ surface brightness plotted against galactic properties for the galaxies in this study. The galactic properties in this figure were those measured in the regions defined on the pressure maps. The top panel contains these four galaxies plus 25 more from the LITTLE THINGS sample plotted against galactic pressure. In the top panel the pressure was measured in an annulus $200 \mathrm{pc}$ wide located just beyond the $\mathrm{H}$ II region. With the larger sample, we do see a correlation: as galactic pressure increases, the $\mathrm{H}$ II region $\mathrm{H} \alpha$ surface brightness also increases. There is no correlation between diameter of the $\mathrm{H} \mathrm{II}$ region and pressure. We would expect the $\mathrm{H} \alpha$ surface brightness to be determined by the concentration of massive stars and gas. Hence, this suggests that higher concentrations of massive stars and gas are preferentially found in regions with higher pressure.

\subsection{OB Associations}

The OB associations are outlined in Figures 8-12, and their properties are given in Table 3 . These objects are large and loose associations of $\mathrm{O}$ stars, which are distinct from the cluster catalogs' class 3 objects that are compact associations. One can see from the summary of total numbers given in Table 2 that DDO 50, DDO 53, and DDO 63 have more OB associations than clusters. Even DDO 63, which has no clusters, has six OB associations. Hence, for these galaxies, OB associations appear to be a better descriptor of the mode of star formation in these systems. NGC 3738 and Haro 29 are different, perhaps because they are more extreme in SFR over all. Haro 29 has a comparable number of clusters and OB associations, 9 and 7, respectively, and NGC 3738 has 138 clusters and three OB associations. In addition, the OB associations in NGC 3738 and four of those in Haro 29 have a higher stellar mass density than those in the other three galaxies. OB association \#3 in NGC 3738 has a density that is 12 times higher than the highestdensity region in DDO 50, DDO 53, or DDO 63. OB association \#1 in NGC 3738 is very large, encompassing half of the optical galaxy within 0.5 disk scale length radius (Hunter et al. 2018).

The equivalent of Figure 13 is shown for the OB associations in Figure 23. The OB association characteristics of stellar mass and mass surface density are plotted against environmental characteristics of pressure and H I surface density. We see, again, that $\mathrm{OB}$ associations in NGC 3738 and Haro 29 are more massive and have a higher mass surface density than those found in the other three galaxies. This is unlikely to be a consequence of their further distances since the OB associations are all highly resolved. The associations in NGC 3738 


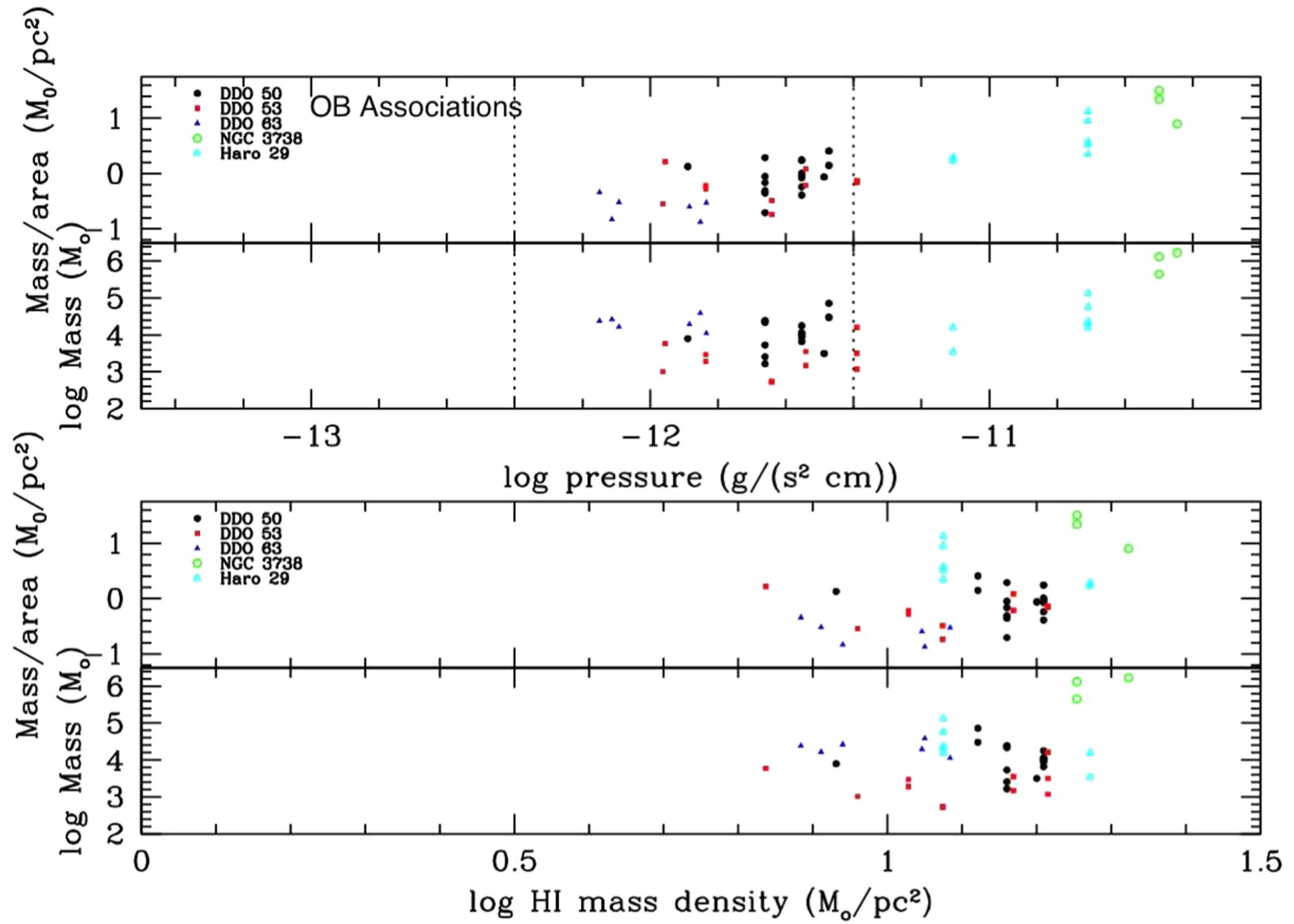

Figure 23. OB association characteristics of stellar mass and mass surface density vs. environmental characteristics of pressure and $\mathrm{H}$ I surface density. The vertical dotted lines in the top panel delineate the three pressure bins discussed in the text, and the $x$-axis is the same as that of Figure 13 for clusters. We see that the OB associations in Haro 29 and NGC 3738 are more extreme in mass and mass density than those in the other three dIrrs, and they are found at the highest pressure.

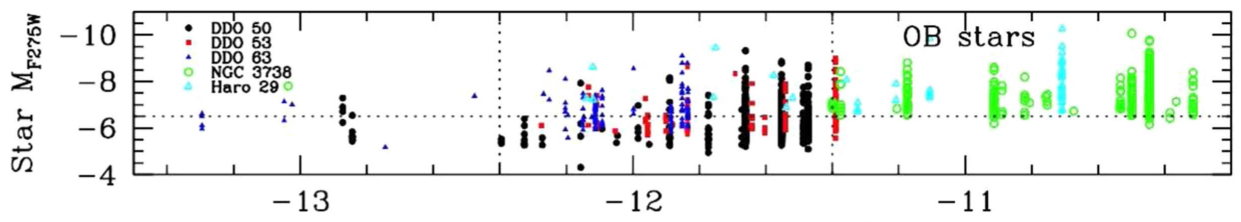

$\log$ pressure $\left(\mathrm{g} /\left(\mathrm{s}^{2} \mathrm{~cm}\right)\right)$
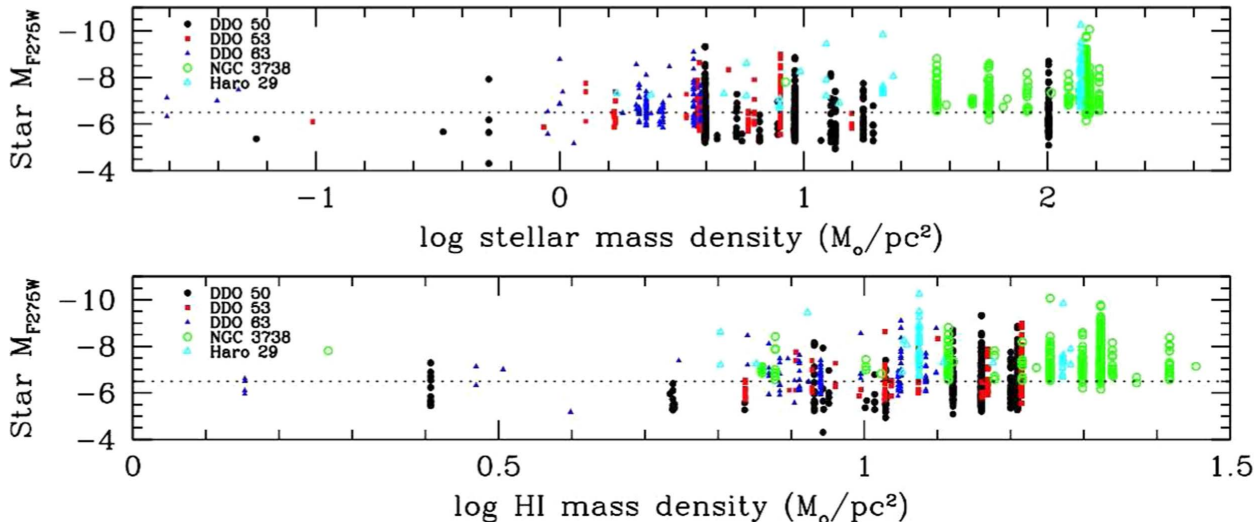

Figure 24. O star F275W absolute magnitude vs. galactic environment in which the stars are found. Galactic environmental characteristics include pressure, stellar mass density, and H I mass surface density. The vertical dotted lines in the top panel delineate the three pressure bins discussed in the text, and the $x$-axis is the same as that of Figure 13 for clusters. The variations in lower limits from galaxy to galaxy are likely due to distance effects and incompleteness due to the higher backgrounds in the higher SFR galaxies. The horizontal dashed line at an $M_{\mathrm{F} 275 \mathrm{~W}}$ of -6.5 delineates an absolute magnitude cutoff that is common to all of the galaxies. Note that we have ignored differential extinction within each galaxy and variations of average extinction between galaxies.

and Haro 29 are also found at higher pressure and mostly at higher HI surface density. The OB associations in DDO 50, DDO 53, and DDO 63 have similar masses and mass densities and are all found in the intermediate-pressure bin. The OB associations of DDO 53 and DDO 63 tend to be found at lower H I densities. 


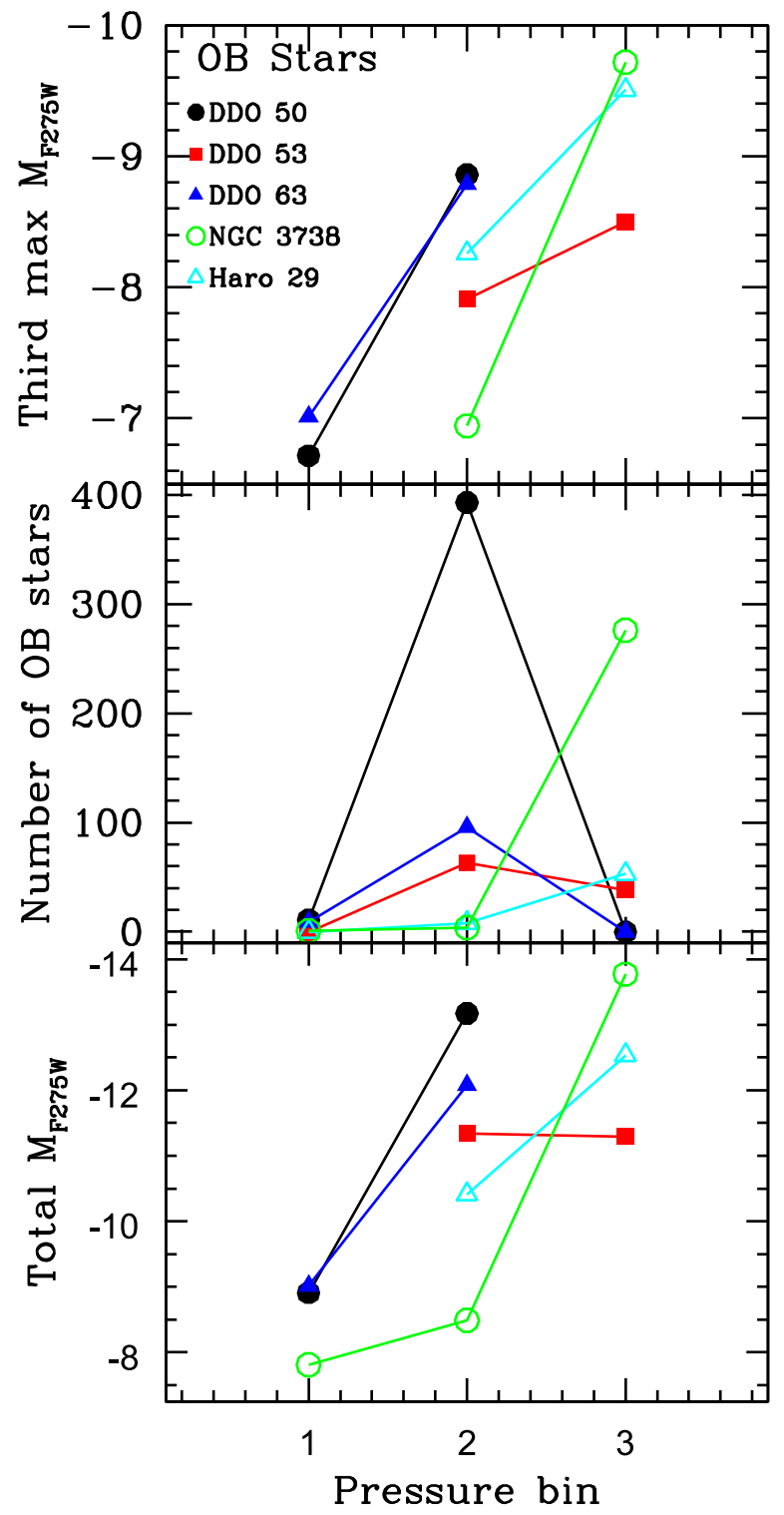

Figure 25. O star characteristics by pressure bin: third-brightest absolute F275W magnitude, number of stars, and integrated stellar F275W absolute magnitude. The pressures fall in three bins: bin 1 is log pressure $<-12.4$, bin 2 is $\log$ pressure between -12.4 and -11.4 , and bin 3 is $\log$ pressure $>-11.4$. Units of pressure are $\mathrm{g}\left(\mathrm{s}^{2} \mathrm{~cm}\right)^{-1}$.

\subsection{O Star Distributions}

Here we turn our attention from star clusters and $\mathrm{OB}$ associations to individual $\mathrm{O}$ stars. The distributions of the candidate $\mathrm{O}$ stars are shown in Figures 8-12. Not all stars are captured in the selected OB associations. This does not necessarily imply that some $\mathrm{O}$ stars have formed in isolation, although that is possible, but could imply that our recognition of OB associations, especially small or older associations, may be inadequate. The stellar characteristics that we have to work with are absolute $\mathrm{F} 275 \mathrm{~W}$ magnitude $M_{\mathrm{F} 275 \mathrm{~W}}$ and number of stars.

\subsubsection{Characteristics as a Function of Galactic Characteristics}

In Figure 24 we plot the $\mathrm{O}$ star equivalent of Figure 13: stellar $M_{F 275 W}$ against the three environmental characteristics pressure, stellar mass density, and H I mass density. First, we see that $\mathrm{O}$ stars are found at a wide range of pressures, including the lowest-pressure bin 1 where no clusters are found, as we saw for the OB associations that contain most of the $\mathrm{O}$ stars. Furthermore, the $\mathrm{O}$ stars are not coincident with the clusters. This suggests that $\mathrm{O}$ stars in these dwarfs are preferentially formed in less compact units that are perhaps not bound.

Second, we see that, like the clusters and $\mathrm{OB}$ associations, $\mathrm{O}$ stars at high pressure are found exclusively in NGC 3738 and Haro 29, and $\mathrm{O}$ stars at high stellar mass density and gas mass density are mostly found in NGC 3738 and Haro 29. Furthermore, most of the O stars in NGC 3738 and Haro 29 are found in regions with high densities.

Third, like the properties of clusters and OB associations, generally $\mathrm{O}$ star magnitudes cover a large range regardless of pressure or density. One exception is that $\mathrm{O}$ stars in all galaxies do not extend to the same faintness level. However, the lower limits correlate with the distance to the galaxy: DDO 50 (3.1 Mpc) stars extend to $M_{\mathrm{F} 275 \mathrm{~W}}$ of $\sim-5$, DDO $53(3.7 \mathrm{Mpc})$ stars extend to $\sim-5.5$, DDO $63(4.0 \mathrm{Mpc})$ stars extend to $\sim-6$, NGC 3738 (4.9 Mpc) stars extend to $\sim-6$, and Haro 29 (5.9 $\mathrm{Mpc}$ ) stars extend to $\sim-6.5$. Therefore, the change in the lower limits is to some extent a distance effect, with the more distant galaxies having brighter absolute magnitude limits. In addition, incompleteness due to higher backgrounds in the higher SFR galaxies may also play a role. The horizontal dashed line in Figure 24 marks a stellar absolute magnitude in F275W, $M_{\mathrm{F} 275 \mathrm{~W}}$, of -6.5 . To see the effect of making an absolute magnitude lower limit cutoff to the stellar catalogs, look only at the stars above this line. Doing this, one can see that the stars in pressure bins 2 and 3 extend to more or less the same upper magnitude. However, the brightest stars are found in NGC 3738 and Haro 29 in pressure bin 3, but there are only a few of these, and they could be due to blending at the further distances of these galaxies. Also, at the low-pressure side of the figure, bin 1 , there are no stars brighter than about -7.5 , although there are also fewer stars in this pressure bin. Both of these effects could be due to size-of-sample effects in that the SFR is also a function of the pressure with the higher-SFR galaxies having many more $\mathrm{O}$ stars than the lower-SFR galaxies or regions (see Whitemore 2017).

To correct the $\mathrm{F} 275 \mathrm{~W}$ photometry of the $\mathrm{O}$ stars for extinction, we applied a modest constant correction for internal extinction of $E(B-V)=0.05 \mathrm{mag}$ per galaxy. This corresponds to an $A_{V}$ of $0.16 \mathrm{mag}$. This value is also fairly consistent with $E(B-V)$ of $\mathrm{OB}$ associations determined from SED fitting and given in Table 3. Additional extinction evenly distributed across a galaxy would only cause us to underestimate the luminosity of all $\mathrm{O}$ stars in that galaxy by the same factor. However, differential extinction across the galaxy would affect the intercomparison of stars. Kahre et al. (2018) have developed a method of mapping the extinction in LEGUS galaxies by determining the reddening for each object from its photometry in the galaxy's stellar catalog (see Sabbi et al. 2018). We have looked at the extinction map and stellar extinction histogram for NGC 3738 as likely the worst case in our sample of galaxies. NGC 3738 has dust lanes that are clearly visible in color images in a small region to the north of the center of the galaxy, at an R.A. and decl. centered around $11^{\mathrm{h}} 35^{\mathrm{m}} 49^{\mathrm{s}} .0,54^{\circ} 31^{\prime} 34^{\prime \prime}$. The extinction map indeed shows that this region has the highest extinction, up to $A_{V} \sim 1.2$, and this 

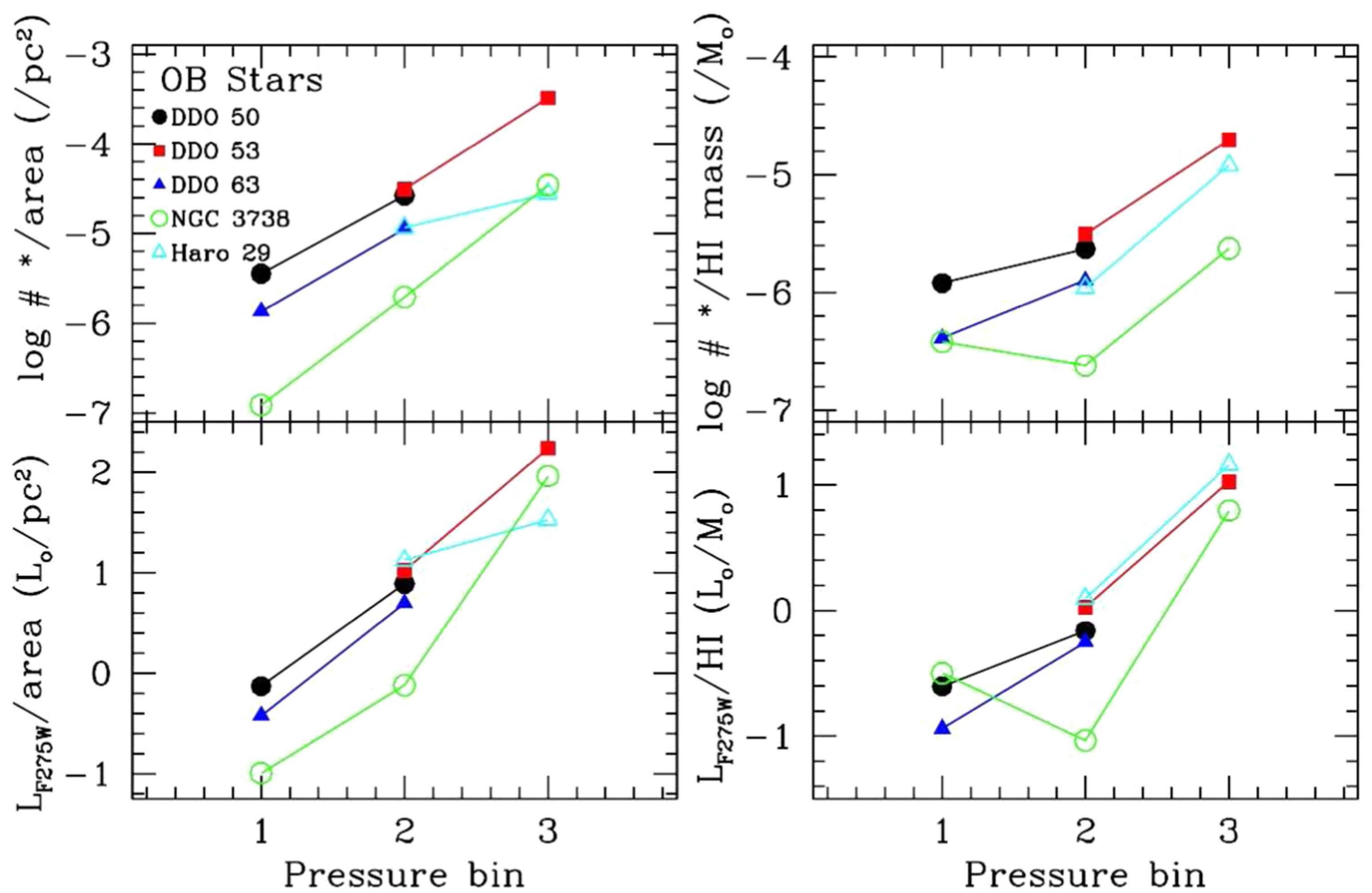

Figure 26. Number and total F275W luminosity of O stars per unit area and per H I gas mass vs. pressure in which the stars are found. This is similar to Figure 18, but for $\mathrm{O}$ stars. The pressures fall in three bins: bin 1 is $\log$ pressure $<-12.4$, bin 2 is $\log$ pressure between -12.4 and -11.4 , and bin 3 is $\log$ pressure $>-11.4$. Units of pressure are $\mathrm{g}\left(\mathrm{s}^{2} \mathrm{~cm}\right)^{-1}$, units of area are pc ${ }^{2}$, units of mass are $M_{\odot}$, and units of F275W luminosity are $L_{\odot}$. The area is the total area of pressure regions shown in Figure 7 within the given bin, and similarly for the H I mass. Only NGC 3738 has O stars in all three pressure bins.

region contains of order 10 clusters and $\mathrm{O}$ stars used in this study. However, a histogram of stellar extinctions shows that most stars have $A_{V}$ near zero with a tail to higher $A_{V}$ that involves a relatively small number of stars. Thus, while we have underestimated the absolute $\mathrm{F} 275 \mathrm{~W}$ magnitude of some of the stars in NGC 3738 by up to 2 mag, the numbers that are affected are small. Furthermore, since the region of heaviest extinction is also at the highest pressure, these very luminous stars would only accentuate the trends that we see in that galaxy (see Section 3.4.2).

\subsubsection{Characteristics by Pressure Region}

In Figure 25 we examine the $\mathrm{O}$ star characteristics by pressure bin, including the third-brightest $M_{\mathrm{F} 275 \mathrm{~W}}$, number of $\mathrm{O}$ stars, and $M_{\mathrm{F} 275 \mathrm{~W}}$ integrated over all of the $\mathrm{O}$ stars in the pressure bin. We choose to plot the third-brightest star rather than the brightest in order to reduce statistical scatter from using the single brightest star. We see that the third-brightest $M_{\mathrm{F} 275 \mathrm{~W}}$ and integrated $M_{\mathrm{F} 275 \mathrm{~W}}$ are generally higher at higher pressure, but only in NGC 3738 is the number of O stars significantly higher in the highest-pressure bin.

In Figure 26 we plot the $\mathrm{O}$ star characteristics per unit area and per unit $\mathrm{H}$ I gas mass by pressure bin, similar to Figure 18. We see that the number of $\mathrm{O}$ stars and integrated F275W flux per unit area and per unit $\mathrm{HI}$ gas mass increase with pressure.

Figure 27 is similar to Figure 20, but for O stars. Here we plot the integrated F275W flux relative to the SFR by pressure bin. We see that generally the higher the pressure, the higher the O star F275W flux per unit SFR. Similarly, Figure 25 showed that the maximum absolute F275W magnitude of the $\mathrm{O}$ stars increases with pressure bin. By contrast, in Figure 20

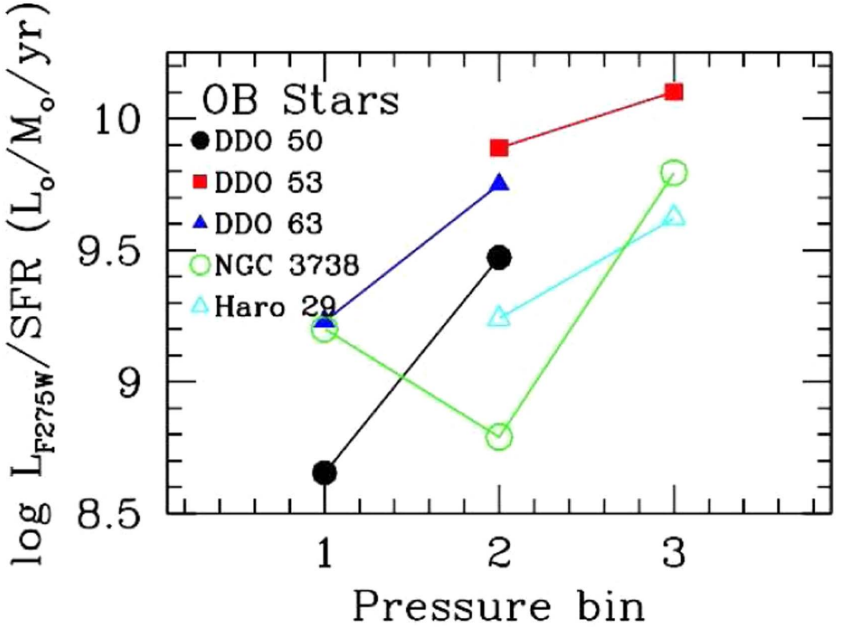

Figure 27. Total O star F275W luminosity divided by the SFR by pressure bin, similar to Figure 20 for clusters. The pressures within each galaxy fall in three bins: bin 1 is $\log$ pressure $<-12.4$, bin 2 is $\log$ pressure between -12.4 and -11.4 , and bin 3 is $\log$ pressure $>-11.4$. Units of pressure are $\mathrm{g}\left(\mathrm{s}^{2} \mathrm{~cm}\right)^{-1}$, units of SFR are $M_{\odot} \mathrm{yr}^{-1}$, and units of F275W luminosity are $L_{\odot}$. Only NGC 3738 has $\mathrm{O}$ stars in all three pressure bins.

Haro 29 has flat values of integrated cluster mass divided by $\mathrm{SFR}$ with pressure bin. The $\mathrm{O}$ star cluster difference may imply that the mass of the most massive star increases with pressure or that the ratio between ongoing star formation and star formation averaged over the past $100 \mathrm{Myr}$ increases with pressure.

In the left panels of Figure 28, we examine the relative number of clusters and $\mathrm{O}$ stars. The top two panels show a 

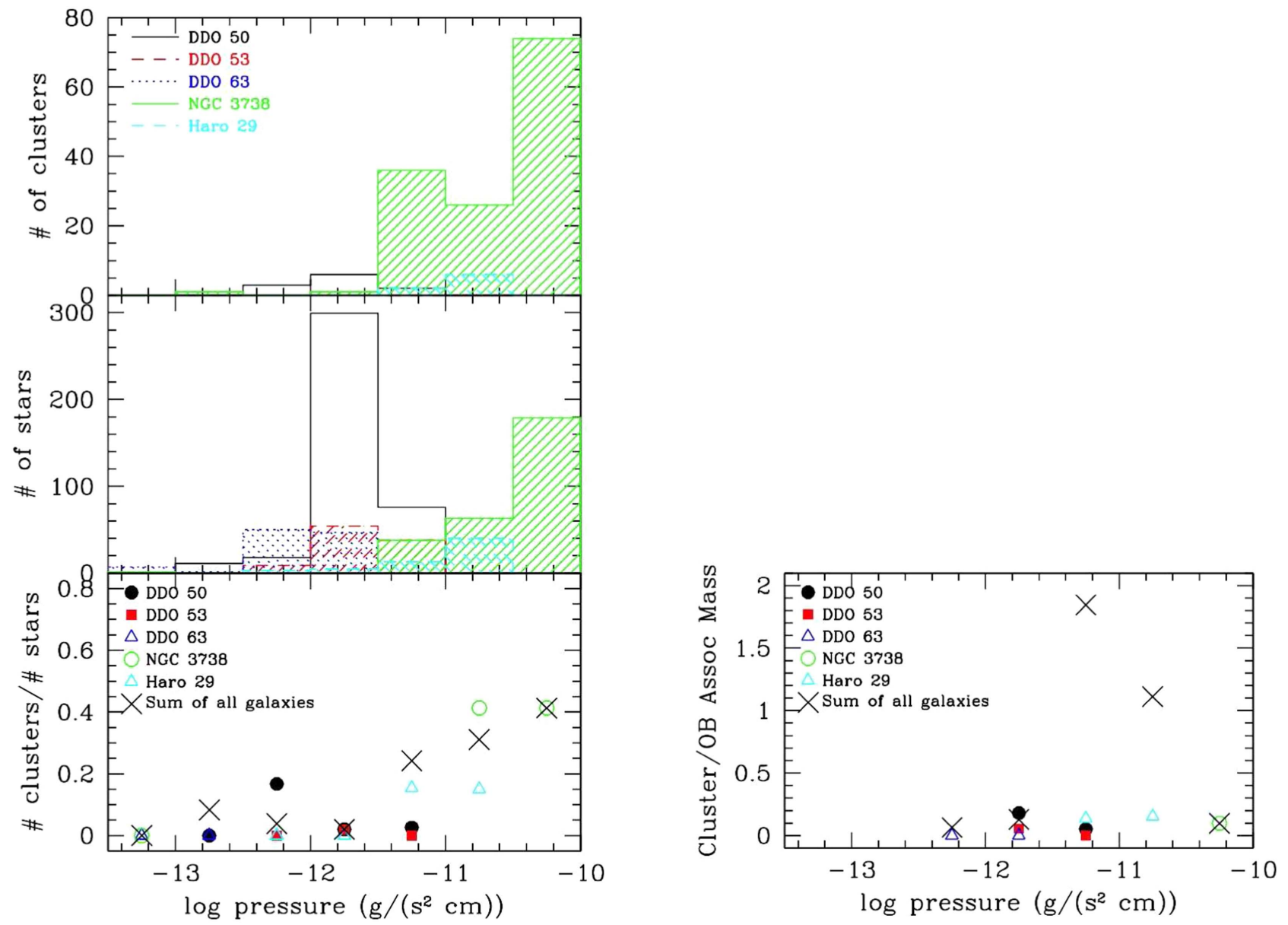

Figure 28. Left: number of clusters, number of $\mathrm{O}$ stars, and the ratio of clusters to $\mathrm{O}$ stars plotted against the pressure binned by 0.5 in the logarithm. The large black crosses in the bottom panel come from adding all of the clusters and stars in each pressure bin in all five galaxies and taking the ratio; it is not the average of the individual galaxy ratios. Right: total mass of clusters divided by the total mass of OB associations, vs. pressure bin. For a given pressure bin, a galaxy might have clusters but no OB associations or vice versa. The large black crosses in the bottom panel come from adding the mass of all of the clusters and OB associations in each pressure bin in all five galaxies.

histogram of each separately. The bottom panel shows the ratio of clusters to $\mathrm{O}$ stars. Here we have summed in $0.5 \mathrm{log}$ pressure bins. The large black crosses are the ratios of the sums of clusters and $\mathrm{O}$ stars in all five galaxies. We see that the ratio increases with pressure.

Here the clusters are small compact clusters or tiny associations that may be bound, while the $\mathrm{O}$ stars are primarily grouped into physically larger associations. For a given stellar IMF, the number of $\mathrm{O}$ stars formed in the clusters is just a scaling factor times the cluster mass. Therefore, the ratio of number of clusters to number of $\mathrm{O}$ star candidates is related to the ratio of the number of $\mathrm{O}$ stars formed in clusters to the number formed in larger $\mathrm{OB}$ associations. This relationship is messy because the clusters are not all the same mass and the number of $\mathrm{O}$ stars in a particular association declines with time. Nevertheless, the rise of the ratio of number of clusters to number of $\mathrm{O}$ star candidates with pressure could indicate that the number of $\mathrm{O}$ stars formed in clusters compared to the number formed in associations increases with pressure. This is in spite of the fact that the relationship is dominated at the high end by NGC 3738, where half of the central part of the galaxy is one giant $\mathrm{OB}$ association.

On the right side of Figure 28 we plot the sum of the mass in clusters divided by the sum of the mass in $\mathrm{OB}$ associations by pressure for each galaxy (for pressures that have at least one OB association). This may be related to the amount of star formation taking place in bound systems relative to that taking place in unbound systems. We find that the ratio of cluster mass to $\mathrm{OB}$ association mass is fairly low and fairly flat. When masses are summed over all galaxies (the large black crosses in Figure 28), the high values at $\log$ pressure -11.25 and -10.75 are also driven by NGC 3738 , which has one very large OB association that is assigned to log pressure -10.4 .

\section{Summary}

In order to examine the role environmental factors play in determining characteristics of typical star-forming units, we present a comparison of the concentrations, masses, and formation rates of young ( $\leqslant 100 \mathrm{Myr}$ ) compact star clusters, surface brightnesses of $\mathrm{H}$ II regions, masses and mass surface densities of large and loose OB associations, and distributions and $\mathrm{F} 275 \mathrm{~W}$ magnitudes of candidate $\mathrm{O}$ stars with surrounding galactic pressure, stellar mass density, H I surface density, and SFR surface density. Our sample consists of three dIrr galaxies and two BCD-like galaxies within $5.9 \mathrm{Mpc}$. For H II region characteristics we include an additional 25 dIrrs and BCDs from the LITTLE THINGS sample. We find the following: 
1. The BCD galaxies are more extreme than the dIrrs: most of their clusters, $\mathrm{OB}$ associations, and $\mathrm{O}$ star candidates are found at higher pressures, stellar mass densities, and $\mathrm{HI}$ mass densities. In addition, NGC 3738 has an extraordinary $\mathrm{OB}$ association that occupies half of the inner part of the galaxy (see Hunter et al. 2018). Both of these galaxies may be dwarf-dwarf mergers (Ashley et al. 2013, 2017), and perhaps such events are necessary to produce large numbers of clusters or extraordinary regions in dwarfs.

2. There is no trend of cluster characteristics with environmental properties, implying that larger-scale effects are more important in determining cluster characteristics (e.g., Whitmore et al. 2007) or that rapid dynamical evolution (such as stellar feedback, merging of subclumps in the young cluster, and tidal stripping by the host galaxy) is taking place in bound stellar systems that erases any memory of the initial conditions.

3. The most massive OB associations are found at higher pressure and $\mathrm{H}$ I surface densities, and there is a trend of higher $\mathrm{HII}$ region $\mathrm{H} \alpha$ surface brightness with higher pressure, suggesting that a higher concentration of massive stars and gas is found preferentially in regions of higher pressure. Furthermore, the SFR per unit area increases with pressure.

4. The number of clusters and total mass in clusters per unit area and per unit gas mass generally increase with pressure, while the mass formed in clusters divided by the SFR is approximately a constant with pressure.

5. $\Gamma$, the ratio of cluster formation rate to SFR, does not show a correlation with the total SFR density, but the galaxies studied here do scatter in $\Gamma$ around the sequence of $\Gamma$ versus SFR density found in other galaxies.

6. O star candidates are found at a wide range of pressures, including low pressures where bound clusters and $\mathrm{OB}$ associations are not found.

7. The number of candidate $O$ stars and the integrated F275W flux per unit area and per unit $\mathrm{HI}$ gas mass increase with pressure. Furthermore, the total $\mathrm{O}$ star F275W flux per unit SFR and the third-brightest absolute F275W magnitude of an $\mathrm{O}$ star increase with pressure. This may imply that the mass of the most massive star increases with pressure or that the ratio between ongoing star formation and star formation averaged over the past 100 Myr increases with pressure.

8. The ratio of the number of clusters to number of $\mathrm{O}$ star candidates increases with pressure, perhaps reflecting an increase in clustering properties with SFR.

Results presented here are based on observations made with the NASA/ESA Hubble Space Telescope under the LEGUS survey. Support for HST program no. 13364 was provided by NASA through a grant from the Space Telescope Science Institute, which is operated by the Association of Universities for Research in Astronomy, Incorporated, under NASA contract NAS5-26555. A.A. acknowledges the support of the Swedish Research Council (Vetenskapsrdet) and the Swedish National Space Board (SNSB). S.G. appreciates funding from the National Science Foundation grant AST-1461200 to Northern Arizona University for Research Experiences for Undergraduates summer internships and Drs. Kathy Eastwood and David Trilling for running the NAU REU program in 2016.
S.G. also appreciates the support of the 2016 CAMPARE Scholar program and Dr. Alexander Rudolph for directing that program.

Facilities: HST(ACS, WFC3), VLA, Lowell Observatory.

\section{ORCID iDs}

Deidre A. Hunter (1) https://orcid.org/0000-0002-3322-9798

Angela Adamo (iD https://orcid.org/0000-0002-8192-8091

Bruce G. Elmegreen (iD https://orcid.org/0000-00021723-6330

Janice C. Lee (iD https://orcid.org/0000-0002-2278-9407

David O. Cook (1D https://orcid.org/0000-0002-6877-7655

David Thilker (1D https://orcid.org/0000-0002-8528-7340

Hwihyun Kim (10 https://orcid.org/0000-0003-4770-688X

Lauren Kahre (iD https://orcid.org/0000-0002-2037-3096

Daniela Calzetti ib https://orcid.org/0000-0002-5189-8004

Monica Tosi (iD https://orcid.org/0000-0002-0986-4759

Kathryn Grasha (1) https://orcid.org/0000-0002-3247-5321

Michele Fumagalli ib https://orcid.org/0000-0001-6676-3842

Daniel A. Dale (i) https://orcid.org/0000-0002-5782-9093

Elena Sabbi (ib https://orcid.org/0000-0003-2954-7643

Michele Cignoni (i) https://orcid.org/0000-0001-6291-6813

Linda J. Smith (1D https://orcid.org/0000-0002-0806-168X

Dimitrios M. Gouliermis (ib https://orcid.org/0000-00022763-0075

Eva K. Grebel (ib https://orcid.org/0000-0002-1891-3794

Alessandra Aloisi (iD https://orcid.org/0000-0003-4137-882X

Rupali Chandar (iD https://orcid.org/0000-0003-0085-4623

Kelsey E. Johnson (i) https://orcid.org/0000-0001-8348-2671

\section{References}

Adamo, A., \& Bastian, N. 2015, in The Origin of Stellar Clusters, ed. S. Stahler (Berlin: Springer), arXiv:1511.08212

Adamo, A., Kruijssen, J. M. D., Bastian, N., Silva-Villa, E., \& Ryon, J. 2015, MNRAS, 452, 246

Adamo, A., Östlin, G., Zackrisson, E., et al. 2010, MNRAS, 407, 870

Adamo, A., Östlin, G., Zackrisson, E., et al. 2011, MNRAS, 415, 2388

Adamo, A., Ryon, J. E., Messa, M., et al. 2017, ApJ, 841, 131

Annibali, F., Tosi, M., Aloisi, A., \& van der Marel, R. P. 2011, AJ, 142, 129

Ashley, T., Simpson, C. E., \& Elmegreen, B. G. 2013, AJ, 146, 42

Ashley, T., Simpson, C. E., Elmegreen, B. G., Johnson, M., \& Pokhrel, N. R. 2017, AJ, 153, 132

Ashman, K. M., \& Zepf, S. E. 2001, AJ, 122, 1888

Aversa, A. G., Johnson, K. E., Brogan, C. L., Goss, W. M., \& Pisano, D. J. 2011, AJ, 141, 125

Baumgardt, H., Parmentier, G., Anders, P., \& Grebel, E. K. 2013, MNRAS, 430, 676

Bernard, E. J., Ferguson, A. M. N., Barker, M. K., et al. 2012, MNRAS, 426, 3490

Bertin, E., \& Arnouts, S. 1996, A\&AS, 117, 393

Bigiel, F., Leroy, A., Walter, F., et al. 2010, AJ, 140, 1194

Billett, O. H., Hunter, D. A., \& Elmegreen, B. G. 2002, AJ, 123, 1454

Blitz, L., \& Rosolowsky, E. 2004, ApJL, 612, L29

Blitz, L., \& Rosolowsky, E. 2006, ApJ, 650, 933

Boquien, M., Kennicutt, R., Calzetti, D., et al. 2016, A\&A, 591, A6

Calzetti, D., Armus, L., Bohlin, R. C., et al. 2000, ApJ, 533, 682

Calzetti, D., Lee, J. C., Sabbi, E., et al. 2015, AJ, 149, 51

Chabrier, G. 2003, ApJ, 586, 133

Chandar, R., Fall, S. M., \& Whitmore, B. C. 2015, ApJ, 810, 1

Chandar, R., Fall, S. M., Whitmore, B. C., \& Mulia, A. J. 2017, ApJ, 849, 128

Cignoni, M., Sacchi, E., Aloisi, A., et al. 2018, ApJ, 856, 62

Cox, D. P. 2005, ARA\&A, 43, 337

da Silva, R. L., Fumagalli, M., \& Krumholz, M. R. 2014, MNRAS, 444, 3275

Dobbs, C. L., Adamo, A., \& Few, C. G. 2017, MNRAS, 464, 3580

Dolphin, A. E., \& Hunter, D. A. 1998, AJ, 116, 1275

Egorov, O. V., Lozinskaya, T. A., Moiseev, A. V., \& Shchekiinov, Y. A. 2017, MNRAS, 464, 1833

Elmegreen, B. G. 1989, ApJ, 338, 178

Elmegreen, B. G. 2017, ApJ, 836, 80 
Elmegreen, B. G., \& Efremov, Y. 1997, ApJ, 480, 235

Elmegreen, B. G., \& Hunter, D. 2015, ApJ, 805, 145

Elmegreen, B. G., \& Hunter, D. A. 2000, ApJ, 540, 814

Elmegreen, B. G., \& Parravano, A. 1994, ApJL, 435, L121

Elmegreen, D. M., \& Elmegreen, B. G. 1980, AJ, 85, 1325

Escala, A., \& Larson, R. B. 2008, ApJL, 685, L31

Evans, N. 1999, ARA\&A, 37, 311

Fall, S. M., \& Chandar, R. 2012, ApJ, 752, 96

Fitzpatrick, E. L. 1999, PASP, 111, 63

Fumagalli, M., da Silva, R. L., Krumholz, M., \& Bigiel, F. 2011, ApJL, 741, L26

Goddard, Q. E., Bastian, N., \& Kennicutt, R. C. 2010, MNRAS, 405, 857

Grasha, K., Calzetti, D., Adamo, A., et al. 2015, ApJ, 815, 93

Grasha, K., Calzetti, D., Adamo, A., et al. 2017, ApJ, 840, 113

Grudić, M. Y., Guszejnov, D., Hopkins, P. F., et al. 2017, MNRAS, submitted (arXiv:1708.09065)

Hao, C.-N., Kennicutt, R. C., Johnson, B. D., et al. 2011, ApJ, 741, 124

Herrmann, K. A., Hunter, D. A., Zhang, H.-X., \& Elmgreen, B. G. 2016, AJ, 152,177

Hoessel, J. G., Saha, A., \& Danielson, G. E. 1998, AJ, 115, 573

Hunter, D. A. 1995, in Revista Mexicana de Astronomia y Astrofisica Serie de Conf. 3: The Fifth Mexico-Texas Conf. Astrophysics: Gaseous Nebulae and Star Formation, ed. M. Pena \& S. Kurtz, 1

Hunter, D. A. 2001, ApJ, 559, 225

Hunter, D. A., \& Elmegreen, B. G. 2004, AJ, 128, 2170

Hunter, D. A., \& Elmegreen, B. G. 2006, ApJS, 162, 49

Hunter, D. A., Elmegreen, B. G., \& Ludka, B. C. 2010, AJ, 139, 447

Hunter, D. A., Ficut-Vicas, D., Ashley, T., et al. 2012, AJ, 144, 134

Hunter, D. A., Gallardo, S., Elmegreen, B. G., et al. 2018, ApJ, 855, 7

Hunter, D. A., O’Neil, E. J., Jr., Lynds, R., et al. 1996, ApJL, 459, L27

Hunter, D. A., \& Thronson, H. A., Jr. 1995, ApJ, 452, 238

Jacobs, B. A., Rizzi, L., Tully, R. B., et al. 2009, AJ, 138, 332

Jarrett, T., Masci, F., Tsai, C.-W., et al. 2012, AJ, 144, 68

Johnson, K. E., Leitherer, C., Vacca, W. D., \& Conti, P. S. 2000, AJ, 120, 1273

Johnson, K. E., Leroy, A. K., Indebetouw, R., et al. 2015, ApJ, 806, 35

Johnson, L. C., Seth, A. C., Dalcanton, J. J., et al. 2016, ApJ, 827, 33

Kahre, L., Walterbos, R. A., Kim, H., et al. 2018, ApJ, 855, 133

Karachentsev, I. D., Sharina, M. E., Dolphin, A. E., et al. 2003, A\&A, 398, 467

Kennicutt, R. C., \& Chu, Y.-H. 1988, AJ, 95, 720

Kroupa, P. 2001, MNRAS, 322, 231

Kruijssen, J. M. D. 2012, MNRAS, 426, 3008
Kruijssen, J. M. D. 2015, MNRAS, 454, 1658

Krumholz, M. R., Fumagalli, M., da Silva, R. L., Rendahl, T., \& Parra, J. 2015, MNRAS, 452, 1447

Lada, C. J. 2010, RSPTA, 368, 713

Lamers, H. J. G. L. M. 2009, Ap\&SS, 324, 183

Lang, D. 2014, AJ, 147, 108

Lim, S., \& Lee, M. G. 2015, ApJ, 804, 123

Longmore, S. N., Kruijssen, J. M. D., Bastian, N., et al. 2014, in Protostars and Planets VI, ed. H. Beuther et al. (Tucson, AZ: Univ. Arizona Press), 291

Lynds, R., Tolstoy, E., O’Neil, E. J., Jr., \& Hunter, D. A. 1998, AJ, 116, 146 Maíz-Apellániz, J. 2001, ApJ, 563, 151

McQuinn, K. B. W., Skillman, E. D., Dolphin, A. E., \& Mitchell, N. P. 2015, ApJ, 808, 109

Meisner, A. M., Lang, D., \& Schlegel, D. J. 2017, AJ, 153, 38

O'Connell, R. W., Gallagher, J. S., III, \& Hunter, D. A. 1994, ApJ, 433, 65

Parmentier, G., Pfalzner, S., \& Grebel, E. K. 2014, ApJ, 791, 132

Peek, J. E. G., \& Schiminovich, D. 2013, ApJ, 771, 68

Press, W. H., Teukosky, S. A., Vetterling, W. T., \& Flannery, B. P. 2007, Numerical Recipes. The Art of Scientific Computing (3rd ed.; Cambridge: Cambridge Univ. Press), 779

Reines, A. E., Johnson, K. E., \& Hunt, L. K. 2008, AJ, 136, 1415

Renaud, F., Bournaud, F., Emsellem, E., et al. 2015, MNRAS, 454, 3299

Ryon, J. E., Adamo, A., Bastian, N., et al. 2014, AJ, 148, 33

Ryon, J. E., Gallagher, J. S., Smith, L. J., et al. 2017, ApJ, 841, 92

Sabbi, E., Calzetti, D., Ubeda, L., et al. 2018, ApJS, 235, 23

Schlafly, E. F., \& Finkbeiner, D. P. 2011, ApJ, 737, 103

Schulte-Ladbeck, R. E., Hopp, U., Greggio, L., Crone, M. M., \& DrozDovsky, I. O. 2001, AJ, 121, 3007

Simpson, C. E., \& Gottesman, S. T. 2000, AJ, 120, 2975

Swaters, R. A. 1999, PhD Dissertation, Rijksuniversiteit Groningen

Swinbank, A. M., Smail, I., Longmore, S., et al. 2010, Natur, 464, 733

Tully, R. B., Courtois, H. M., Dolphin, A. E., et al. 2013, AJ, 146, 86

Whitemore, B. C. 2017, in IAU Symp. 316, Formation, Evolution, and Survival of Massive Star Clusters, ed. C. Charbonnel \& A. Nota (Cambridge: Cambridge Univ. Press), 202

Whitmore, B. C., Chandar, R., \& Fall, S. M. 2007, AJ, 133, 1067

Whitmore, B. C., \& Schweizer, F. 1995, AJ, 109, 960

Youngblood, A. J., \& Hunter, D. A. 1999, ApJ, 519, 55

Zackrisson, E., Rydberg, C.-E., Schaerer, D., Östlin, G., \& Tuli, M. 2011, ApJ, 740,13

Zhang, H.-X., \& Puzia, T. H. 2017, ApJS, 233, 13 